\title{
Behavioural and Neurochemical \\ Consequences of MDMA Self-administration in Rats
}

by

Jennifer Do

A thesis submitted in fulfillment of the requirements for the degree of Doctor of Philosophy

Victoria University of Wellington 


\section{Acknowledgements}

I would like to acknowledge the continuous support of my parents, brothers, sister, friends and Fletcher. Up in the lab, most of my thanks would have to go to Richard Moore, who was always happy to assist and Dr. Joyce Colussi-Mas, who very patiently taught me many of the skills I needed. My experience up in the lab was made much more pleasant due to the upbeat attitude of my co-workers, and for that I'm thankful. A special thanks to Judith.

I have learnt a great deal during this experience and for that I'm grateful to my supervisor, Prof. Sue Schenk.

I would also like to acknowledge the Royal Society of New Zealand which provided funding for this project through a Marsden grant. This also assisted with living expenses throughout the course of my PhD studies. 


\section{Contents}

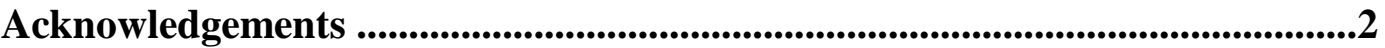

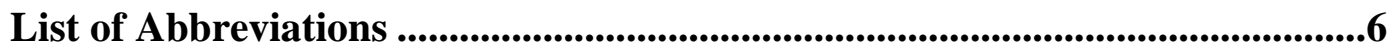

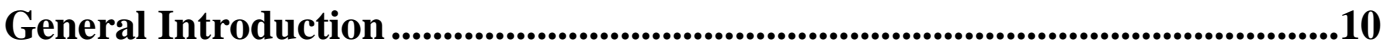

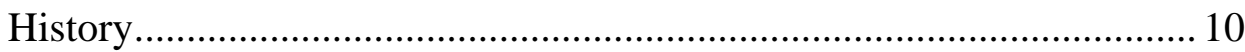

MDMA pharmacology \& pharmacokinetics......................................... 11

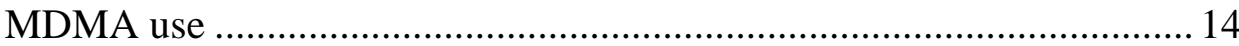

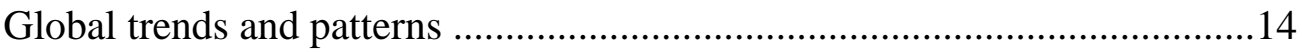

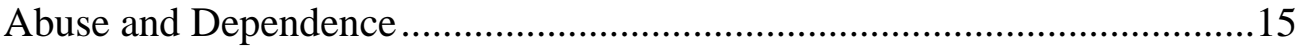

Long-term effects of Ecstasy use; changes in global brain activity and the

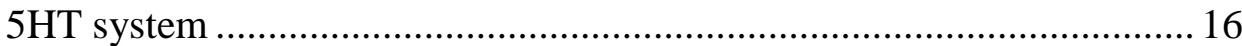

Long-term behavioural effects of Ecstasy use: Cognition ....................... 19

Long-term effects of MDMA on cognition: animal studies ................... 21

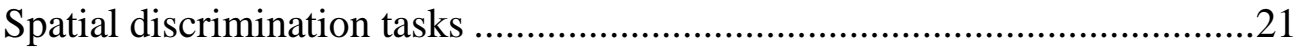

Object/visual stimulus discrimination tasks..............................................24

Novel Object Recognition......................................................................24

Long-term effects of MDMA in laboratory animals: Brain tissue 5HT levels

Acute effects of MDMA on tissue levels of 5HT ......................................28

The effects of chronic exposure to MDMA on tissue levels of 5HT

Neurotoxicity vs Neuroadaptation 32

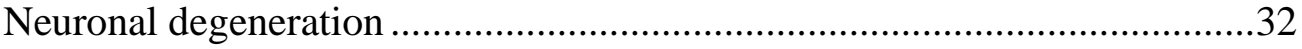

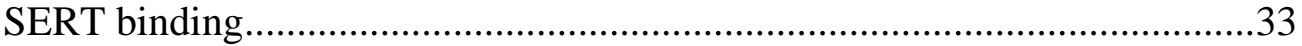

Mechanisms of MDMA-induced decrease in tissue 5HT levels: $5 \mathrm{HT}_{1 \mathrm{a}}$ autoreceptor

Mechanisms of MDMA-produced 5HT depletion: the role of tryptophan hydroxylase 39

The use of animal models of drug intake: dose \& "interspecies scaling" 41

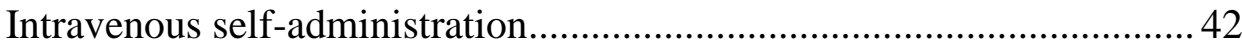

MDMA self-administration .45

Effects of drug self-administration on NOR 47

MDMA self-administration and 5HT 48

The Present study .49 


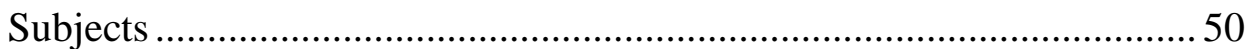

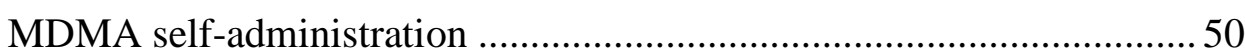

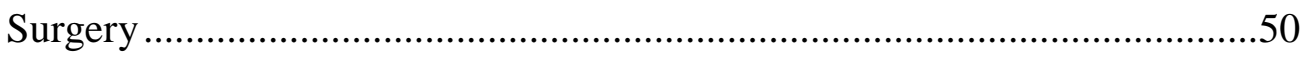

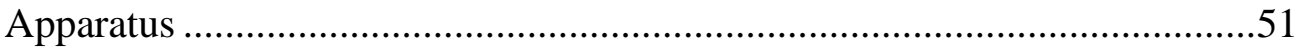

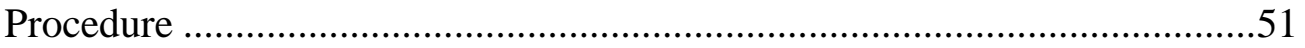

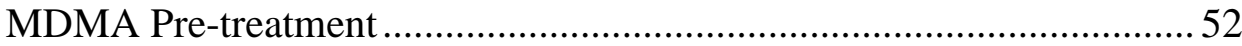

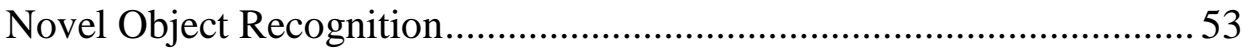

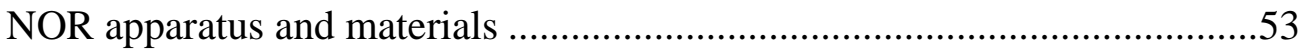

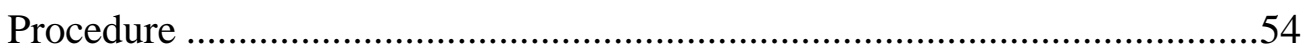

Exploratory behaviour scoring and analysis ...........................................55

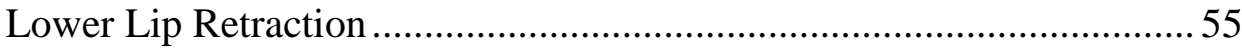

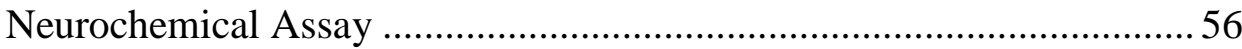

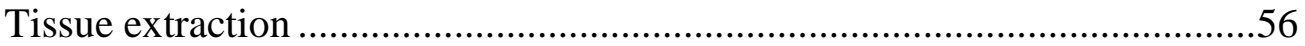

Tissue preparation and HPLC analysis ...................................................56

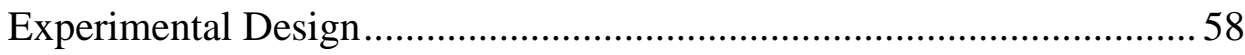

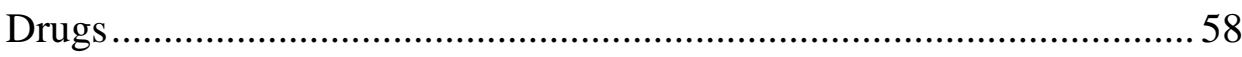

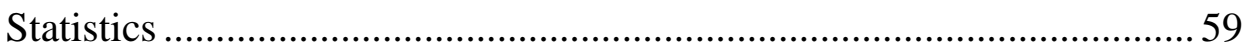

Chapter 3: Preliminary experiments; Establishing the NOR task using short inter-trial intervals in drug naïve-rats ...........................................60

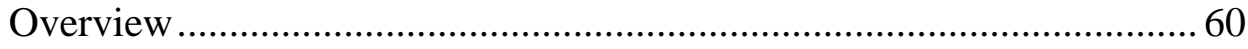

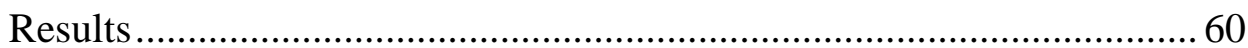

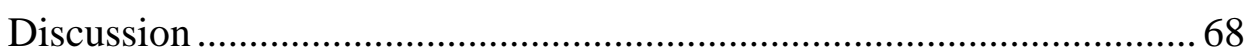

4: The effects of MDMA self-administration on NOR.......................................73

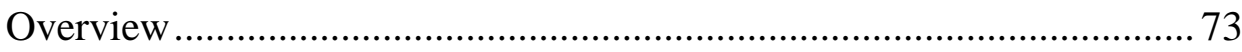

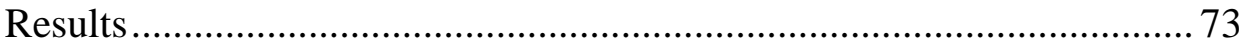

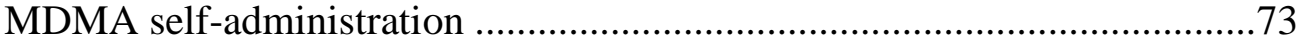

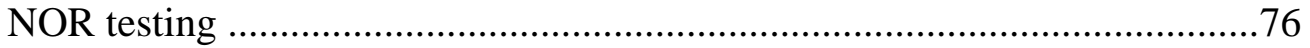

NOR testing 7 days following self-administration ......................................76

NOR testing 70 days following self-administration ...................................79

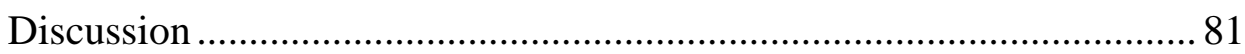

Chapter 5: Dose- and time-dependent effects of MDMA self-

administration on tissue levels of $5 \mathrm{HT}$

Overview 
Results

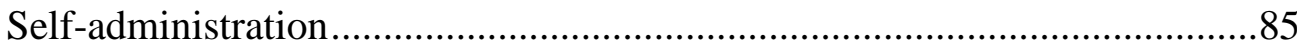

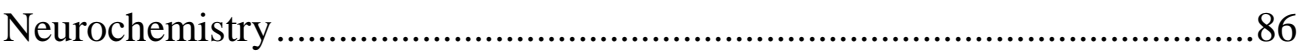

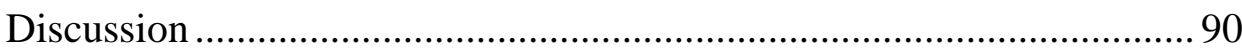

Chapter 6: Mechanisms of 5HT-produced deficits

6.1: The role of the 5HT1a autoreceptor: 8-OH-DPAT-induced lower lip retraction

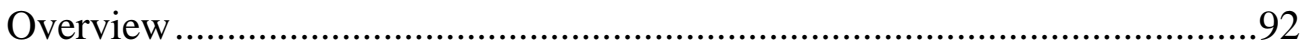

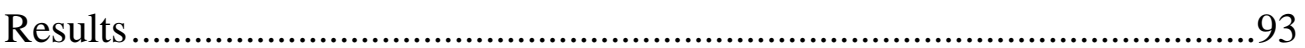

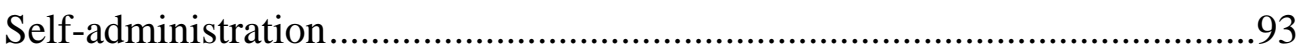

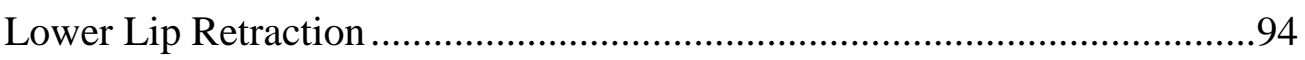

6.2: Effects of chronic 8-OH-DPAT on tissue levels of 5HT following

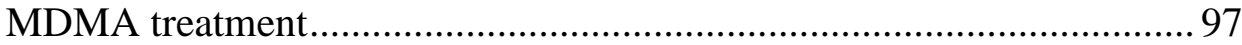

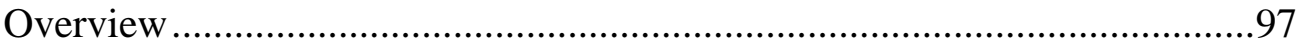

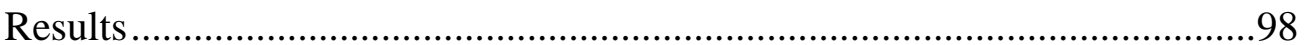

6.3: Effects of chronic trazodone treatment following MDMA exposure102

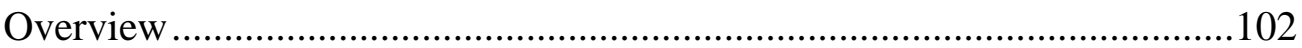

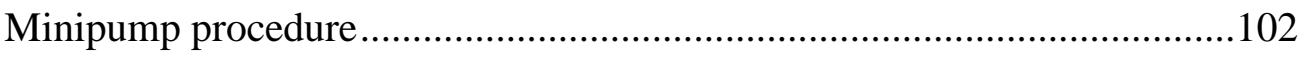

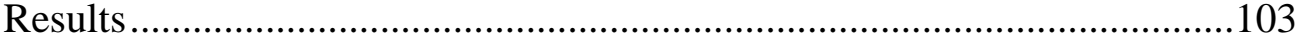

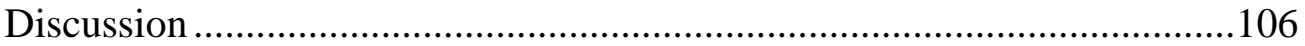

Chapter 7: The role of TPH in MDMA-produced 5HT deficits: Effects of tryptophan loading ................................................................................108

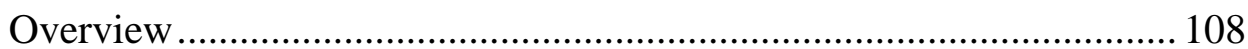

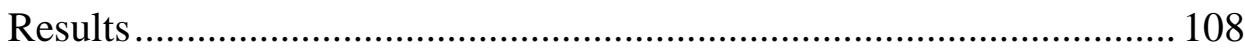

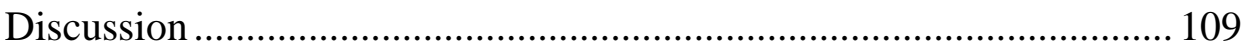

Chapter 8: General Discussion ......................................................................111

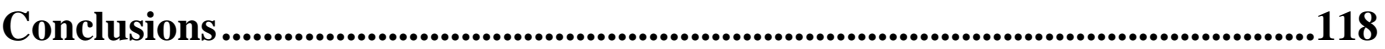

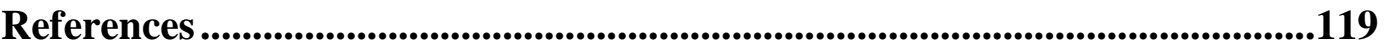




\section{List of Abbreviations}

${ }^{1} \mathrm{H}$ MR: proton magnetic resonance

5,7-DHT: 5, 7-Dihydroxytryptamine

5HT: serotonin

ADC: apparent diffusion coefficients

APP: amyloid precursor protein

Cho: choline

CSF: Cerebrospinal fluid

CWM: Cincinnati water maze

DA: dopamine

DAT: DA transporter blockers

DMTS: delayed-matching-to-sample

DNMP: delayed-non-matching-position

DNMS: delayed-non-matching-to-sample

EEG: electroencephalography

FA: fractional anisotropy

FST: forced swim test

GFAP: glial fibrillary acidic proteins

HHA: \pm 3 ,4-dihydroxyamphetamine

HHMA: \pm 3 ,4-dihydroxymethamphetamine

HMA: \pm 4 -hydroxy-3-methoxyamphetamine

HMMA: \pm 4 -hydroxy-3-

methoxymethamphetamine

MAO: monoamine oxidase

MDA: $\pm 3,4$-methylenedioxyamphetamine

MI: Myo-Inositol

MRI: Magnetic resonance imaging

MWM: Morris water maze

NAA: N-acetylaspartate

NE; norepinephrine

NOR: novel object recognition

RAM: radial arm maze 
rCBF: regional cerebral blood flow

SERT: selective 5HT transporter blockers

SPECT: single photon emission tomography

SSRI: selective 5HT reuptake inhibitors:

TPH: tryptophan hydroxylase

VMAT: vesicular monoamine transporters 


\begin{abstract}
Rationale: Over the past few decades, MDMA has been shown to produce persistent detrimental effects. Animal models have been developed to investigate the effects of self-administered drugs on brain and behaviour, but only a limited number of studies have investigated effects of MDMA. Objectives: The present thesis sought to determine the effects of MDMA self-administration on working memory and tissue levels of 5HT in rats. The role of the $5 \mathrm{HT}_{1 \mathrm{a}}$ autoreceptor in MDMA-produced deficits in tissue levels of 5HT was also evaluated using neurochemical and behavioural assays.
\end{abstract}

Methods: Rats self-administered a total of $165 \mathrm{mg} / \mathrm{kg}$ MDMA, and were then tested in the Novel Object Recognition (NOR) task 1 week or 9 weeks following the last session of MDMA self-administration. Tissue levels of 5HT were measured in separate groups of rats, following self-administration of a total dose of $165 \mathrm{mg} / \mathrm{kg}$ or $315 \mathrm{mg} / \mathrm{kg}$. 8-OH-DPAT-induced lower lip retraction (LLR) was measured in rats 2 weeks following either selfadministered $(315 \mathrm{mg} / \mathrm{kg})$ or experimenter-administered $(40 \mathrm{mg} / \mathrm{kg})$ MDMA. In subsequent studies, chronic 8-OH-DPAT (daily injections over 7 days; $1.0 \mathrm{mg} / \mathrm{kg} / \mathrm{day}$ ), chronic trazodone (continuous infusion over 14 days via osmotic minipump; $10 \mathrm{mg} / \mathrm{kg} /$ day) and tryptophan loading (oral administration over 7 days; $125 \mathrm{mg} /$ day via gavaging needle) were administered after MDMA treatment (either self-administered; $315 \mathrm{mg} / \mathrm{kg}$ or experimenter-administered; $40 \mathrm{mg} / \mathrm{kg}$ ) and tissue levels of $5 \mathrm{HT}$ were measured.

Results: Self-administered MDMA produced deficits in NOR that recovered 10 weeks following self-administration. There was a small decrease in tissue levels of $5 \mathrm{HT}$ at both 2 weeks and 10 weeks following the low dose of self-administered MDMA. Two weeks following the high dose, tissue levels of $5 \mathrm{HT}$ were decreased by about $30 \%$ in all brain regions examined, and there was recovery 10 weeks following exposure. 8-OH-DPAT-induced LLR was unchanged in MDMA-treated rats. Furthermore, none of the treatments restored tissue levels of 5HT following MDMA exposure, even 
though the treatment (chronic 8-OH-DPAT) shifted the basal 8-OH-DPATinduced LLR curve to the right, suggesting autoreceptor desensitisation. Conclusions: Self-administered MDMA produced deficits in NOR, which may reflect impaired attention, encoding, novelty seeking or other cognitive processes. Dose- and time-dependent deficits in tissue levels of 5HT were modest compared to those produced by experimenter-administered MDMA. Therefore, MDMA self-administration may be important for pre-clinical investigation of long-term consequences of MDMA. The findings are not consistent with the idea that the $5 \mathrm{HT}_{1 \mathrm{a}}$ autoreceptor became supersensitive as a result of MDMA exposure, and it is therefore not a viable pharmacological target for restoring tissue levels of 5HT. 


\section{General Introduction}

\section{History}

MDMA (3,4-methylenedioxymethamphetamine), street name Ecstasy, is a recreational drug which was originally synthesised and patented by Merck in 1912 (Freudenmann et al, 2006). Pharmacological testing was first carried out by the company in 1927 and later in the 1950s (Freudenmann et al, 2006). It was not until the 1970s that the effects of MDMA on the human psyche were investigated and found to be favourable for its potential clinical use in psychotherapy (Bezenhofer \& Passie, 2010). MDMA produced a feeling of being 'touched within' which led to its initial classification as an 'entactogen' (Nichols, 1986). This property made it potentially useful as an adjunct in psychotherapy but also made it attractive for recreational use. A USA survey conducted in 1986 showed that this unique ability to produce feelings of love, peace and happiness was reported as one of the main reasons MDMA was used recreationally (Cohen et al, 1995).

While use in the psychiatric field was being explored, its popularity as a recreational drug gained momentum in the United States and abroad, eventually becoming synonymous with the 'dance' or 'rave' scene (Pentney, 2001). Widespread infiltration into society during this time prompted its scheduling in the UK and MDMA became illegal in 1977. Several years later, the Drug Enforcement Administration (DEA) followed suit and in 1985 it was classed as a schedule 1 drug in the United States. MDMA is legally controlled and criminalised in many parts of the world today although use and persist. 


\section{MDMA pharmacology \& pharmacokinetics}

MDMA produces a rapid and robust elevation of extracellular serotonin (5HT), dopamine (DA) and norepinephrine (NE) in the brain (Green et al, 2003). These effects on synaptic monoamine levels are produced by direct interaction with monoamine transporters and the vesicular monoamine transporters (VMAT; Rudnick \& Wall, 1992; Bogen et al, 2003). Co-administration with selective 5HT (SERT) and DA (DAT) transporter blockers (for example, citalopram, fluoxetine, amfonelic acid) attenuated MDMA-induced efflux of monoamines, suggesting an important role of monoamine transporters (Schmidt et al, 1987; Gudelsky \& Nash, 1996; Hekmatpanah \& Peroutka, 1990; Gu \& Amitzia, 1993; Berger et al, 1992).

MDMA acts as a substrate for the monoamine transporter, causing release of monoamines into the synaptic cleft through transporter-mediated exchange (Berger et al, 1992; Hekmatpanah \& Peroutka, 1990; Yamamoto \& Spanos, 1988; Rudnick \& Wall, 1992; Fitzgerald \& Reid, 1990). Furthermore, MDMA stimulates release of vesicular 5HT into the cytoplasm and disrupts the sequestration processes, thereby preventing 5HT uptake into vesicular stores. These effects are achieved through direct interaction with the VMAT (Rudnick \& Wall, 1992; Bogen et al, 2003). Indeed, depletion of vesicular 5HT stores by resperpine attenuated MDMAinduced increase in extracellular monoamines (Gu \& Amitzia, 1993; Fitzgerald \& Reid, 1993). MDMA also inhibits monoamine oxidase (MAO), further contributing to increased extracellular levels of monoamines ( $\mathrm{Gu} \&$ Amitzia, 1993). Therefore, MDMA produces efflux of monoamine into the synapse through a dual mechanism; it increases cytoplasmic levels of monoamines by interacting with the VMAT, and causes release of monoamines into the synapse through transporter-mediated exchange.

MDMA is ubiquitous and interacts with numerous brain recognition sites, but binds with the highest affinity to SERT (Battaglia et al, 1988b). MDMA increased synaptic 5HT, DA and NE in rat synaptosomal preparations and brain slices (Nichols et al, 1982; Schmidt et al, 1987; 
Johnson et al, 1986; Bogen et al, 2003; Fitzgerald \& Reid, 1990; Rothman et al, 2001), and was more potent in releasing 5HT than DA (Fitzgerald \& Reid, 1993; McKenna et al, 1991). Numerous in vivo microdialysis studies have corroborated this (Gough et al, 1991; Yamamoto \& Spanos, 1988; Hiramatsu et al, 1991; Nash \& Nichols, 1991; Reveron et al, 2010; Estaban et al, 2001; Baumann et al, 2008; Sabol \& Seiden, 1998), and demonstrated the preferential effect of MDMA on 5HT (Reveron et al, 2010; Baumann et al, 2008; 2008b; Gudelsky \& Nash, 1996; Kehr et al, 2012).

MDMA produces large dose-dependent increases in synaptic 5HT, and to a lesser extent, DA. A low dose of $1.5 \mathrm{mg} / \mathrm{kg}$ that increased extracellular 5HT levels, failed to increase DA levels, but a higher dose of $7.5 \mathrm{mg} / \mathrm{kg}$ that increased 5HT levels 30-fold increased DA levels 5-fold (Baumann et al, 2008). Following a self-administered intravenous (i.v.) injection of MDMA (3mg/kg), extracellular levels of $5 \mathrm{HT}$ increased to about $1200 \%$ of baseline, and extracellular DA levels peaked at $500 \%$ of baseline (Reveron et al, 2010).

MDMA is consumed as a racemic mixture of the (R)-enantiomer and the (S)-enantiomer in a 1:1 ratio (Pizarro et al, 2004) and is metabolised mainly in the liver. Biotransformation occurs through two different pathways (Figure 1.2; Baumann et al, 2009). In the major pathway, MDMA is converted to \pm 3 ,4-dihydroxymethamphetamine (HHMA) via Odemthylenation by the CYP2D6 enzyme in humans and the CYP2D1 enzyme in rats. HMMA is then O-methylated to form \pm 4 -hydroxy-3methoxymethamphetamine (HMMA). In the minor pathway, MDMA is Ndemethylated to form \pm 3 ,4-methylenedioxyamphetamine (MDA). The CYP2D6 and CYP2D1 enzymes then convert MDA to $\pm 3,4-$ dihydroxyamphetamine (HHA) and this is O-methylated to \pm 4 -hydroxy-3methoxyamphetamine (HMA). There are notable differences in metabolism between humans and rats, and within rat strains, with respect to the enzyme isoform responsible for O-demthylenation of MDMA and MDA (See Figure 1. and Tucker et al, 1994; Kumangi et al, 1994). These species-related differences in drug metabolism bear significance for interspecies scaling of doses between humans and laboratory animals, as discussed later. 


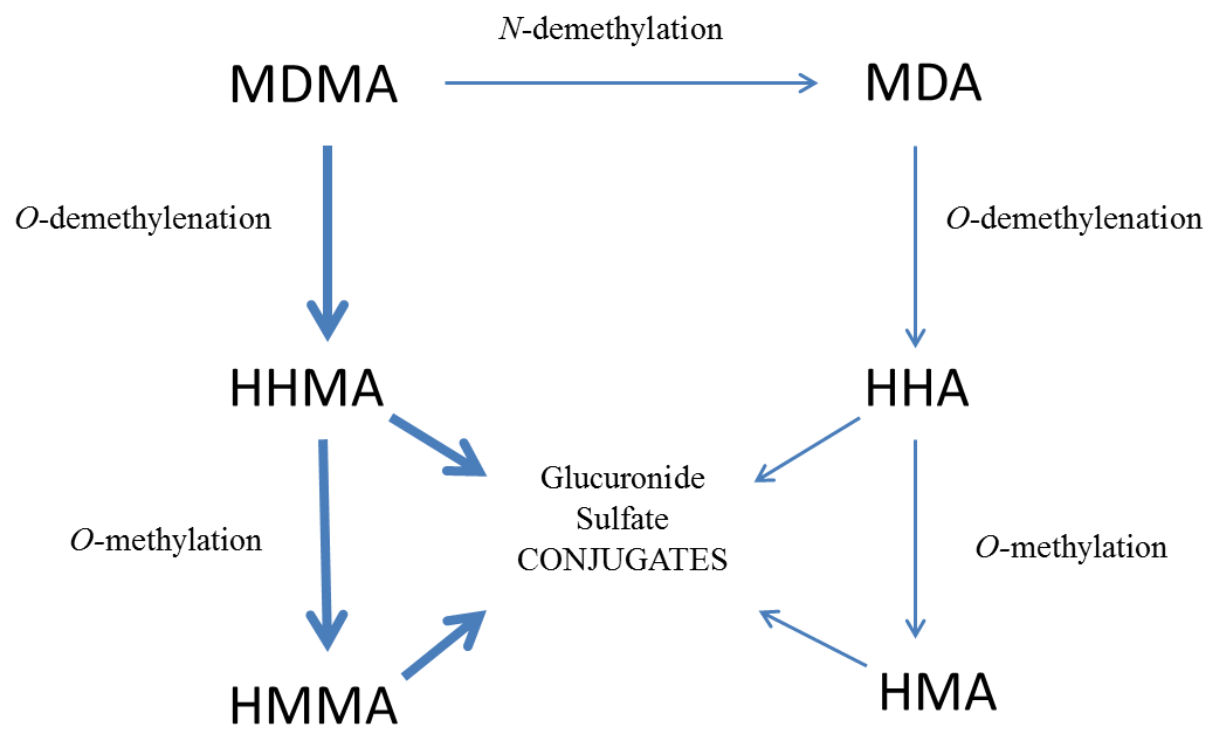

Figure 1.2 Major (bold arrows) and minor metabolic pathways of MDMA in both humans (H) and rats (R). (Adapted from Baumann et al, 2009).

The pharmacokinetics of MDMA have been determined in humans under controlled experimental conditions (De la Torre et al, 2000; Farre et al, 2004; Kolbrich et al, 2008; Mas et al, 1999; Mueller et al, 2009; Pizarro et al, 2004; Fallon et al, 1999). A disproportionally greater rise in MDMA concentration was produced by increasing dose within the range that are used recreationally (De la Torre et al, 2000; Kolbrich et al, 2008; Mas et al, 1999; Mueller et al, 2009; Farre et al, 2004). This pattern of non-linearity was also observed in non-human primates following administration of doses estimated to be equivalent to those consumed recreationally (Mueller et al, 2008; 2009; Mechan et al, 2006).

In rats, non-linear pharmacokinetics was not evident following administration of low doses of MDMA (i.e. $1.0 \mathrm{mg} / \mathrm{kg}$; Hirt et al, 2010) but emerged following administration of higher doses $(5.0 \mathrm{mg} / \mathrm{kg}-40 \mathrm{mg} / \mathrm{kg}$; Chu et al, 1996; Baumann et al, 2009). The difference in propensity for nonlinearity between rats and humans has been suggested to reflect the different isoforms of the enzyme; CYP2D6 and CYP2D1. Non-linear pharmacokinetics was attributed to mechanism-based inhibition of CYP2D6/1, which refers to the conversion of MDMA by CYP2D6/1 to a 
substrate that can irreversibly inhibit CYP2D6/1(Heydari et al, 2004). This would explain the exponential accumulation of MDMA with increasing dose.

An important difference in the pharmacokinetics of MDMA between species is the elimination $\mathrm{T}_{1 / 2}$. The $\mathrm{T}_{1 / 2}$ of MDMA in humans following recreationally relevant doses has been estimated to be 6-10 hours (Mueller et al, 2009; Mas et al, 1999; Kolbrich et al, 2008; Farre et al, 2004). In rats, the $\mathrm{T}_{1 / 2}$ of MDMA following a $2 \mathrm{mg} / \mathrm{kg}$ dose was 45 minutes, while following $10 \mathrm{mg} / \mathrm{kg}$, the $\mathrm{T}_{1 / 2}$ increased to more than 1 hour (Baumann et al, 2009). In non-human primates, the $T_{1 / 2}$ of $2.8 \mathrm{mg} / \mathrm{kg}$ (which produces the same peak concentration as $1.6 \mathrm{mg} / \mathrm{kg}$ in humans) was 2 hours compared to 8 hours in humans. The more rapid clearance of MDMA combined with the lower propensity for non-linear kinetics suggests that it is difficult, if not impossible, to achieve the exact same pharmacokinetic profile of MDMA using the same dosing conditions across species (Mueller et al, 2009). Due to the rapid clearance of MDMA in rats and non-human primates, repeated dosing regimens may be an acceptable alternative to a single dose schedule in humans (Baumann et al, 2009).

\section{$\underline{\text { MDMA use }}$}

\section{Global trends and patterns}

Between 11 and 28 million people are estimated to have used ecstasy in 2009 , equating to a prevalence rate of $0.2-0.6 \%$ of the population aged between 15 and 64 (World Drug Report, Annual Report 2011). In Europe, the prevalence of ecstasy use of 15 - 64 year-olds was $3.3 \%$ or 11 million people (EMCDDA 2010, Annual Report 2010). In North America (current prevalence rate of approximately 1.1\%), Ecstasy use decreased between 2006 and 2008, and then increased from 2008 to 2009 (World Drug Report, 2011). The highest prevalence of Ecstasy consumption is found in 
Oceania, with Australia and New Zealand reporting prevalence rates of 4.2\% and $2.6 \%$, respectively. Illicit drug use in Oceania is hallmarked by the high prevalence rates of amphetamine-type stimulants (Ecstasy, cocaine and amphetamine), and the increase in use during the past decade, especially in 13-45 year olds (Wilkins \& Sweetsur, 2008). Expert perception forecasts that prevalence will continue to increase (World drug report, 2011).

\section{Abuse and Dependence}

Elation of mood as well as the ability to connect with others are often cited as the main reason for Ecstasy use (White et al, 2006; Cohen et al, 1995; Verheyden et al, 2003; Milroy CM, 1999; Siegel RK, 1986; Peters $\&$ Kok, 2009). Many users believe that abuse or dependence are not factors which influence consumption, with general consensus being that it is impossible to become addicted to or dependent on Ecstasy (Bahora et al, 2009). Instead, the majority of participants cited the unknown content of illicit drugs, and legal consequences, as the risks of Ecstasy use (White et al, 2006; Bahora et al, 2009). Meta-analyses reveal that the legal ramifications are the reason users eventually decreased Ecstasy use (Peters \& Kok, 2009).

When Ecstasy gained popularity as a recreational drug in the early 1990s, binges were uncommon, and long-term users generally consumed ecstasy on a casual or periodical basis (McDowell et al, 1994). Towards the late 1990s and early 2000s, however, surveys revealed that average lifetime use had increased markedly, the number of ecstasy pills consumed in a single session escalated, and use became more frequent (Parrott et al, 2001; McCann et al, 1998; Reneman et al, 2000; Pope et al, 2001). A subgroup of heavy users who consumed large quantities of Ecstasy on a regular basis eventually emerged. Development of tolerance could potentially explain the increase in number of ecstasy pills taken in a single session (Parrot, 2001). Indeed, tolerance is a relatively common self-reported problem associated with use (35-47\% of users; Cottler et al, 2001;2009; Verheyden et al, 2003).

Case studies and surveys have shown that many users meet the DSM-IV criteria for dependence (Cottler et al, 2001; 2009; Jansen et al, 
1999; Abdallah et al, 2007). Withdrawal and 'continued use despite knowledge of physical or psychological problems' were the two most commonly reported criteria (Cottler et al, 2001; 2009). For example, at least half of a sample of Ecstasy users reported withdrawal symptoms related to changes in appetite and increased lethargy, and about $40 \%$ reported higher anxiety, depression, sleeping and concentration problems (Cottler et al, 2001; 2009). Despite a relatively high incidence, withdrawal symptoms are mild compared to those produced by other drugs (e.g. opioids, alcohol; Degenhardt, 2010). Instead, the dependence syndrome consists mostly of psychological factors (Degenhardt, 2010).

The increased prevalence and altered pattern of Ecstasy consumption has serious consequences. For example, many users report depression and loss of concentration as residual feelings following Ecstasy use (Cohen et al, 1986; Verheyden et al, 2003; Curran \& Travil, 1997). Furthermore, some report that relationships with friends and family as well as work and study can suffer following extensive Ecstasy use (Verheyden et al, 2003). These factors may contribute to a diminished quality of life. As a result of self-reported problematic issues associated with Ecstasy use, investigations into the long-term effects of this drug have been undertaken.

\section{Long-term effects of Ecstasy use; changes in global brain activity and the}

\section{HT system}

Changes in brain morphology (Cowan et al, 2003; Chang et al, 2001), and neuronal loss (Reneman et al, 2002) have been reported in Ecstasy users, but some studies suggest that deficits only manifest when use is extensive. Magnetic resonance imaging (MRI), using single photon emission tomography (SPECT), has shown that relatively low levels of Ecstasy use of 1-2 years did not change global brain volume (Chang et al, 2000). In heavier users, however, a decrease in global brain volume, and reductions in grey matter in the neocortex, cerebellum and brainstem were observed (Chang et al, 2000; Cowen et al, 2003). When markers of neuronal 
loss (NAA; N-acetylaspartate) were measured in Ecstasy users using proton magnetic resonance $\left({ }^{1} \mathrm{H} \mathrm{MR}\right)$ spectroscopy, decreased NAA levels in the frontal cortex were observed in users who reported consuming an average of 723 pills (Reneman et al, 2002). These effects were not produced following lower consumption levels (324 pills; Daumann et al, 2004, 75 occasions of use; Chang et al, 1999). Importantly, decreased NAA levels were correlated with extent of use (Reneman et al, 2002). These changes can indicate neurodenegeration, but findings should be interpreted with caution. For example, where effects were more prominent (Cowen et al, 2003), Ecstasy users also reported higher use of all other drug classes (with the exception of alcohol and heroin; Cowan et al, 2003) and therefore, it is difficult to attribute these changes specifically to Ecstasy use.

A number of studies have also investigated brain activity in Ecstasy users by measuring electroencephalography (EEG; Adamaszek et al, 2010; Dafters et al, 1999; Gamma et al, 2001; Bauernfeind et al, 2011) and regional cerebral blood flow (rCBF; Gamma et al, 2000; 2001). When administered subacutely, Ecstasy dose-dependently reduced baseline rCBF 2-3 weeks later (Chang et al, 2000), but other studies investigating rCBF revealed no differences in the brain during a vigilance task (Gamma et al, 2000; 2001). Differences in global resting brain activity was observed in Ecstasy users (Adamaszek et al, 2010; Gamma et al, 2000; 2001; Dafters) and increased cortical excitability was attributed to a loss of serotonergic input to the cortex (Bauernfeind et al, 2011). EEG coherence (measure of synchronisation of firing between two locations in cortex) was negatively correlated with Ecstasy use, although this finding was restricted to the visual cortex (Dafters et al, 1999).

It is impossible to ascertain whether changes in global brain activity and morphology were present prior to Ecstasy use, or were a result of Ecstasy use. A prospective neuroimaging study attempted to address this issue by measuring parameters of neurotoxicity both before and after Ecstasy use. Axonal integrity was assessed by measuring apparent diffusion coefficients (ADC) and fractional anisotropy (FA) of the diffusional motion of water molecules in the brain. Regional differences in FA were observed in Ecstasy users (average of 6 pills over 20 weeks). The decrease in FA in 
the thalamus was purported to reflect axonal damage while that in the frontoparietal cortex was thought to be more indicative of abnormal axonal maturation, as the white matter was general unaffected by MDMA use. Therefore, even low use of Ecstasy can produce changes in brain morphology. To convincingly demonstrate neurotoxicity, changes in NAA, MI or Cho levels were expected. These changes, however, were not observed in this study (De win et al, 2008).

Other studies have investigated 5HT system integrity in both abstinent and current Ecstasy users. There were reductions in SERT-binding in heavy (Buchert et al, 2003; 2004; De Win et al, 2008b; McCann et al, 2005; 2008; Reneman et al, 2001a; 2001b; Semple et al, 1999; Thomasius et al, 2003) but not moderate (Reneman et al, 2001a) users. Importantly, this reduction was correlated with number of Ecstasy pills taken per session (Thomasius et al, 2003). Reductions in SERT binding were no longer apparent in heavy users when testing was conducted at least 1 year following last use (Selveraj et al, 2009; Thomasius et al, 2003; Reneman et al, 2001b; Reneman et al, 2001a; Buchert et al, 2003; 2004), suggesting that recovery occurs. Longitudinal studies have revealed that over the course of follow-up assessment in current users, Ecstasy use declined, but despite continued use, SERT availability normalised(Buchert et al, 2006;

Thomasius et al, 2006). These findings suggest these changes are reversible and that abstinence is not required for recovery. Instead, decreasing Ecstasy use below a critical level may be sufficient for recovery processes to occur.

Other markers of 5HT dysfunction have been measured in Ecstasy users (Ricaurte et al, 1990; McCann et al, 1999; Gerra et al, 2000; Croft et al, 2001; Reneman et al, 2000). For example, $5 \mathrm{HT}_{2 \mathrm{a}}$-receptor binding was higher in the occipital cortex of Ecstasy users (Reneman et al, 2000). When using the intensity dependence of auditory evoked potentials as an index of 5HT function, deficits were observed in Ecstasy users (Croft et al, 2001). Cerebrospinal fluid (CSF) levels of the 5HT metabolite, 5HIAA, were also lower in heavy Ecstasy users (Ricaurte et al, 1990; McCann et al, 1999), reflecting altered 5HT turnover. There was an attenuation of Dfenfluramine-produced corticosterone 3 weeks after last use, but this response recovered after 12 months of abstinence. On the other hand, the 
prolactin response was attenuated both 3 weeks and 12 months following last use, suggesting that some measures of 5HT dysfunction can be persistent (Gerra et al, 2000).

These deficits in 5HT neurotransmission may produce a range of behavioural abnormalities observed in Ecstasy users, include changes in sleep (Fisk \& Montgomery, 2009; Cahart-Harris et al, 2009; Allen et al, 1993), mood (Cohen et al, 1995; MacInnes et al, 2001; Thomasius et al, 2003; Medina \& Shear, 2007; Schilt et al, 2010; McCardle et al, 2004; McCann \& Ricaurte, 1991) and impulsivity (Hanson et al, 2008; Quednow et al, 2007). One well-documented behavioural deficit observed in Ecstasy users is cognitive impairment.

\section{Long-term behavioural effects of Ecstasy use: Cognition}

A wealth of studies have attempted to determine whether Ecstasy use produces impairments in different facets of cognition, including learning, memory, attention, reasoning skills and processing speed (for example, see Krystal, 1992; Fisk et al, 2009; Verdejo-Garcia et al, 2005; Medina \& Shear et al, 2007; McCann et al, 1999; Hoshi et al, 2007; Roberts et al, 2009). Many studies suggest deficits in working memory in both the verbal (Rogers et al, 2009; Bolla et al, 1998; Schilt et al, 2010; Waering et al, 2004; Bhattachary \& Powell, 2001; Thomasius et al, 2003; 2006; Reneman et al, 2000; 200;1 De Sola Llopis et al, 2008; Hoshi et al, 2007; McCann et al, 1999; McCardle et al, 2004; Montgomery et al, 2007; Murphy et al, 2009; Nulsen et al, 2010; Quednow et al, 2006) and visuospatial (De Sola Llopis et al, 2008; Fox et al, 2001; Murphy et al, 2009; Nulsen et al, 2010; Back-Madruga et al, 2003) domain. Additionally, Ecstasy users may experience impaired procedural memory (Blagrove et al, 2011), declarative memory (Blagrove et al, 2011), prospective memory (Hadjiefthyvoulou et al, 2011; Montgomery \& Fisk, 2007), learning (Roberts et al, 2009) and attentional processing (Curran \& Travil, 1997; Indlekofer et al, 2009; McCardle et al, 2004; Zakzanis et al, 2002). Recently, 
a study that assessed performance in a battery of cognitive tasks indicated that impairments became more apparent as the complexity of the task increased (Brown et al, 2010).

Extent of use has considerable influence on Ecstasy-produced cognitive deficits. Performance in cognitive tasks was correlated with lifetime usage (Nulsen et al, 2010; Quednow et al, 2006; Reneman et al, 2001; Verdejo-Garcia et al, 2005; Blagrove et al, 2011; De Sola Llopis et al, 2008; Fox et al, 2001), number of ecstasy pills taken per session (Thomasius et al, 2003), and frequency of use (Back-Madruga et al, 2003). Cognitive deficits were not apparent in moderate users (Indelkofer et al, 2009; Hanson et al, 2010; Halpern et al, 2010; Gouzoulis-Mayfrank et al, 2003), unless testing was conducted shortly (2-3 days) after last use (Blagrove et al, 2011).

The impact of levels of intake and abstinence in Ecstasy-produced cognitive deficits has been assessed by testing in former- and current-users (Hoshi et al, 2003; Reneman et al, 2001; Waering et al, 2004; GouzoulisMayfrank et al, 2003). Verbal working memory deficits were observed in both current and former- heavy users (Waering et al, 2004; Reneman et al, 2001), consistent with reports that Ecstasy-produced deficits in working memory can persist for a relatively long period of time (De Sola et al, 2008; De Sola Llopis et al, 2008; Reneman et al, 2001; Schilt et al, 2010; Waering et al, 2004; Thomasius et al, 2006; Gouzoulis-Mayfrank et al, 2005). Nevertheless, following more prolonged abstinence, even after heavy use, verbal memory was not impaired (Hoshi et al, 2007), suggesting that recovery can occur. Lifetime usage can also influence the persistence of cognitive detriment, as working memory impairments were only observed in heavy MDMA users, when testing was conducted 6-8 months following last use (Gouzoulis-Mayfrank et al, 2003). Additionally, deterioration of memory has been observed with continued use, suggesting a drug-produced effect (Zakzanis \& Young, 2001).

Meta-analyses revealed that when all available data are considered, Ecstasy impaired learning and memory (Laws \& Kokkalis, 2007; Kalechstein et al, 2007; Rogers et al, 2009; Zakzanis et al, 2007). Human studies suggest cognitive deficits, but it is difficult to attribute these deficits 
selectively to Ecstasy use because of a range of confounds. Firstly, the majority of Ecstasy users are likely to be polydrug users (Schifano et al, 1998; Gouzoulis-Mayfrank \& Daumann, 2006). Furthermore, the retrospective nature of many human studies makes it difficult to determine whether detrimental effects occur as a result of Ecstasy use, or reflect a preexisting condition (Reneman et al, 2006; Gouzoulis-mayfrank et al, 2006). In addition, most studies rely on self-reported drug use, which can be inaccurate, and it is impossible to determine the exact quantity of MDMA consumed since the purity of each pill can be highly variable (Cole \& Sumnall, 2003). For these reasons, the results of human studies must be interpreted with caution. Animal studies, however, are an alternative method for investigating the long-term effects of MDMA, and confounds that impact interpretation of human studies are overcome.

\section{Long-term effects of MDMA on cognition: animal studies}

Numerous studies have attempted to examine the effects of MDMA on cognition in animal studies. A number of paradigms have been developed to assess different types of memory processes in laboratory animals and like the human literature, findings suggest that MDMA produces persistent deficits in working memory.

\section{Spatial discrimination tasks}

Because rats are reasonably adept at learning in spatially-related cognitive tests, they have become a popular choice for assessing learning and memory following pharmacological manipulation. Both the acute and long-term effect of MDMA on spatial cognition has been assessed in rats using the Morris water maze (MWM), Cincinnati water maze (CWM), 
radial arm maze (RAM), delayed-non-matching-position (DNMP) task and T-maze (spatial alternation task).

In the MWM, rats are trained to locate a platform in a circular pool of water. To assess cued learning, the platform has a visible marker in close proximity to it (usually mounted above it) and to assess visuo-spatial learning, visual cues are located in the environment surrounding the maze and the platform is hidden. Memory is assessed by removing the platform and measuring time spent in the correct quadrant (where platform was originally located) and proximity scores (proximity to the original location of the platform).

In the CWM, an 'escape' location at the end of the maze must be located, and spatial cognition is assessed by measuring errors (entering the arms of the T-mazes) and latency to find the target. It consists of $9 \mathrm{~T}$-mazes joined together and the stem of the T-mazes forms the main channel of water.

The radial arm maze consists of 8 arms radiating from a centre, and rats are trained to enter 4 of the arms that are baited. Errors include entering a wrong arm or re-visiting a correct arm (Hodges, 1996). The first type of error reflects impairment in reference memory (a memory that is typically used to remember rules), and the second type of error reflects working memory deficits.

In the DNMP task and the spatial alternation task, working memory is measured as correct discrimination between 2 locations. In the beginning of each DNMP task, rats are first placed at a food magazine with two levers on each side (sample and opposite). On presentation of the sample lever, a response initiates the beginning of a variable ITI. Following the ITI, both levers are presented, and a response must be made on the opposite lever. Working memory is measured as the accuracy in responding on the opposite lever. In the spatial alternation task, rats are trained to alternate arm choices in a T-maze. At the start of each session there is forced entry into an open arm (the other arm is blocked), and following a variable ITI, the previously un-entered arm must be entered. The spontaneous spatial alternation task makes use of the spontaneous preference of rats to explore new environments, thus alternating which arm of the T-maze they run into 
(Hughes, 2004). Correct alternation indicates intact working memory (Hughes, 2004). In these tasks, a range of inter-trial intervals (ITI) should be tested to measure the retention interval.

MDMA treatment did not alter performance in the spatial alternation task when testing was conducted 4 days (Cassel et al, 2005) or 4 weeks (Ricaurte et al, 1993) following treatment. The failure to reveal working memory deficits may have been due to the relatively long period between exposure and testing (Ricaurte et al, 1993) or because only a single ITI was tested (Cassel et al, 2005). Indeed, MDMA treatment impaired performance in the DNMP task following the 2 longest ITIs, suggesting working memory deficits (Marston et al, 1999).

When administered 15 minutes prior to training sessions, MDMA dose-dependently impaired working and reference memory in the RAM task (Kay et al, 2010) and visuo-spatial learning in the MWM task (AriasCaveires et al, 2010). Cued-learning, however, was not impaired, suggesting that MDMA altered spatial cognitive processes was specific and not due to disruptions of motivational, visual or motor performance in the task (Arias-Cavieres et al, 2010). Spatial path learning was also impaired in the CWM following MDMA treatment in both adults (Able et al, 2006; Skelton et al, 2008) and adolescents (Skelton et al, 2006).

Repeated MDMA exposure, administered 2 days prior to testing, produced persistent memory deficits in the RAM (Kay et al, 2011). Acquisition of the task was slower in MDMA-treated rats, and when rules were reversed (4 previously correct arms were switched), deficits in recognition were observed. In the MWM, spatial memory deficits were more persistent than learning deficits (Sprague et al, 2003; Camarasa et al, 2008; Able et al, 2006). Spatial learning was impaired when performance in MWM was assessed 4 days following MDMA treatment (Camarasa et al, 2008) but not when testing was conducted 7 days (Sprague et al, 2003) or 23 weeks (Able et al, 2006) following MDMA treatment, Memory deficits, however, were observed in all 3 studies. In another MWM study, the failure to reveal learning and memory deficits 2 weeks after MDMA treatment (Skelton et al, 2008) may have been due to the influence of previous testing in another spatial learning task (Vorhees et al, 2004). When all of the animal 
studies are considered, there is a general impairment of visuo-spatial learning and memory, produced by MDMA exposure (Chummun et al, 2010).

Object/visual stimulus discrimination tasks

Object discrimination tasks like the delayed-matching-to-sample (DMTS), delayed-non-matching-to-sample (DNMS) have been used to assess working memory. Briefly, subjects are presented with an object or visual stimulus (sample) in a first trial. In a second trial, subjects are trained to choose the matching (DMTS) or non-matching (DNMS) stimulus to the sample when presented with 2 choices. During testing, the ability to make the correct choice (working memory) should decay as ITI increases. In pigeons, MDMA treatment produced poorer performance in the DMTS task, after a 5 days withdrawal period, but there was no effects in non-human primates (Frederick et al, 1995; Taffe et al, 2001). In rats, acute MDMA administered 10 minutes prior to sessions, dose-dependently increased percentage errors in the DMTS task (Harper et al, 2005). This impairment in performance was delay-independent and suggests an impairment of encoding or attention, rather than disrupted memory (Harper et al, 2005).

\section{Novel Object Recognition}

Many of the tasks described above require extensive training and therefore are not well suited to investigations addressing the short-term or persistent effects of drugs of abuse. Indeed, observation of differences between drug-exposed and control animals might not be possible when paradigms that require extensive training are employed since a critical period of a deficit might be missed. Therefore, there has been keen interest in adopting a measurement tool that requires little to no training and can be repeatedly administered without interference from previous trials. 
The novel object recognition (NOR) task is a variation of a task which was initially designed to assess cognition in infants (Fagan, 1970; 1974; Rose et al, 1983). It was extended by Ennaceur \& Delacour (1988) to test object recognition memory in rats and capitalizes on the spontaneous exploratory behaviour of rats towards objects. Specifically, it exploits the innate preference exhibited by rats towards novel objects over familiar ones. Thus, when a rat is presented with a novel and familiar object, the majority of exploratory activity is directed towards the novel object. The paradigm involves 2 trials separated by an ITI. In the first trial (T1), 2 identical objects are presented for a fixed duration of time. During the second trial (T2), one of the objects (familiar) from T1 and a novel object are presented. Memory for the familiar object is inferred if exploratory behaviour is directed towards the novel object. Typically, a recognition index (also referred to as a discrimination index) is used as a measure of working memory, and is based on a comparison between exploration time of the 2 objects during T2 (For example see Ennaceur \& Delacour, 1988; Able et al, 2006; Beck et al, 1999; Briand et al, 2008).

NOR for a given ITI can be measured after a single session and subjects can be tested repeatedly. This task does not require any explicit reinforcement or extensive training. Without any requirement of training, this task excludes facets of cognition such as learning or reference memory (Ennaceur \& Delacour, 1988). Because it is a simple task to administer and subjects can be tested repeatedly, NOR has tremendous appeal as a test for assessing the effects of acute and chronic experimental manipulations on memory (Winter et al, 2008). It has been used to assess the effects of a range of drugs of abuse including cocaine (Morrow et al, 2000; 2002; Thompson et al, 2005), methamphetamine (Belcher et al, 2005; 2008; Bisagno et al, 2002; Clark et al, 2007; Marshall et al, 2007; Herring et al, 2008), amphetamine (Ennaceur et al, 1998), cannabis (Quinn et al, 2008; Schneider et al, 2008; Kosiorek et al, 2003) and alcohol (Garcia-Moreno et al, 2002; Brooks et al, 2002; Kim et al, 1997). There are a small number of studies which have made use of the NOR task to assess the effects of MDMA on memory, although results are equivocal. 
There was an effect of high (4 daily injections of $5.0 \mathrm{mg} / \mathrm{kg}$ over two days) but not low (1 daily injection of $5.0 \mathrm{mg} / \mathrm{kg}$ over 2 days) dose MDMA on NOR, 14 weeks following exposure (Morley et al. 2001). Control rats showed $70 \%$ preference for the novel object during exploration in $\mathrm{T} 2$, whereas rats exposed to the high-dose regimen showed about $60 \%$ preference for the novel object, suggesting a small decrease in NOR. The effect was observed following an ITI of 15 minutes, but not 60 minutes, ostensibly due to the lower preference for the novel object in the control group (65\%) following the longer ITI. Absolute exploration times were not provided but $\mathrm{T} 1$ and $\mathrm{T} 2$ were 10 min durations which should have afforded sufficient time for exploration of the objects. Another study (Camarasa et al, 2008) also demonstrated a dose-dependent effect of MDMA when tests were conducted 3 days following MDMA treatment (single dose of $10 \mathrm{mg} / \mathrm{kg}$ and $15 \mathrm{mg} / \mathrm{kg}$ ). However, during T1, total exploration time for both objects was under 10 seconds for both the control and MDMA group, raising concern as to whether there was sufficient exploration to encode the items.

MDMA (4 injections of $15 \mathrm{mg} / \mathrm{kg}$, every 2 hours) failed to impair performance in the NOR task when testing was conducted 30 days following exposure (Able et al, 2006). NOR in the control group was also low (60\% preference for the novel object) suggesting that an ITI of 60 minutes might have been too long (Able et al, 2006). In Listar-hooded rats, however, NOR was apparent following an ITI of 2 hours (Rodsiri et al, 2011), possibly because of the greater visual acuity of this strain. In this study, MDMA (3 injections of $3 \mathrm{mg} / \mathrm{kg}$ or $6 \mathrm{mg} / \mathrm{kg}$ ) dose-dependently impaired NOR when testing was conducted 2 weeks following treatment.

The idea that deficits in NOR are dependent on exposure levels of MDMA was also supported in a study in mice that were treated with either a single or repeated daily injections of MDMA $(10.0 \mathrm{mg} / \mathrm{kg}$; Nawata et al, 2010). Exploration in $\mathrm{T} 1$ was around $20 \mathrm{~s}$ and in $\mathrm{T} 2$ control mice and mice that received the single exposure to MDMA demonstrated NOR. The mice that received repeated exposure to MDMA, however, spent significantly less time exploring the novel item (Nawata et al., 2010). Furthermore, the 
magnitude of the deficit in NOR became more pronounced when the drug free time was increased from 1 to 7 days.

In other studies a high dose of MDMA was administered to rats, twice a day every 5 days from postnatal day (PD) 35-60, in an attempt to mimic weekend binging in adolescents (Piper and Meyer, 2004; Piper et al, 2005). Following a 15 minute ITI, deficits were observed when testing was conducted 1 week following the last injection (Piper \& Meyer, 2004). When the dose was reduced, however, NOR performance, measured following an ITI of either 15 minutes or 30 minutes, was not altered (Piper et al, 2005). In this study, frequency of exploration and duration of exploration was analysed. When exploration time was used, both the MDMA and control group spent more time with the novel object during T2. When frequency of exploration was measured, however, only the control group showed a preference for the novel object, suggesting that effects on NOR might depend on the outcome measure.

Indeed, the use of different behavioural measures of performance in the NOR task may produce different results (Ennaceur et al, 2005). In Wistar and Lister hooded male and female rats, object exploration time, frequency of approaches, latency to first approach and duration of each approach were measured. Latency to first approach proved to be an unreliable measure of NOR. When frequency of approaches, total exploration time and average duration of exploration were analysed, NOR was consistently demonstrated in Listar males and females, but not Wistar females. In Wistar males, NOR was revealed when duration of each exploratory bout and object exploration were analysed, but not when frequency of approaches was analysed. Temporal profiles of behaviour during T2 were also generated by analysing the dependent measures in 3 separate one-minute bins. When the first minute of $\mathrm{T} 2$ was considered, only the use of frequency of approaches as the dependent measure revealed group differences in NOR. Therefore, it is important to analyse more than one dependent measure in order to isolate drug effects.

These studies show that MDMA exposure can impair performance in the NOR task. Because of the failure to test across a range of ITIs, however, performance in the task may not reflect working memory. In 
memory tasks, performance decays as a function of ITI, and experimental manipulations that disrupt memory result in a more rapid decay of this function. ITI-dependent deficits are generally attributed to disrupted memory, and ITI-independent deficits likely reflect attentional or encoding impairments (Chudasama \& Muir, 1997).

$\underline{\text { Long-term effects of MDMA in laboratory animals: Brain tissue 5HT levels }}$

Behavioural deficits have been attributed to neurochemical consequences of MDMA exposure. A reduction of tissue levels of 5HT is the most consistently reported finding. The following sections will detail the effect of acute (single exposure) and chronic (multiple repeat injections) exposure of MDMA on this neurochemical measure.

Acute effects of MDMA on tissue levels of $5 H T$

Following acute MDMA administration, there is a decrease in tissue levels of 5HT; the timecourse and pattern appears to be region-dependent, dose-dependent and also dependent on strain of rat used (Garcia-Osta et al, 2004; Stone et al, 1987; Schmidt, 1987; Schmidt et al, 1987; Gough et al, 1991). Following $10.0 \mathrm{mg} / \mathrm{kg}$ of MDMA, tissue levels of 5HT were measured in multiple brain regions in Sprague-Dawley rats and at different time points (15 minutes-2 weeks) following exposure (Stone et al, 1987). Within an hour, there was a decrease of about $30-40 \%$ across all brain regions examined, and maximal decreases occurred within 3 hours of exposure. Recovery to near control levels (within 12-24 hours) occurred, before a subsequent slow and progressive decline. Two weeks following MDMA administration, tissue levels of 5HT were decreased by 40-50\%. The hypothalamus was less susceptible to the effect of MDMA, and recovery was observed 24 hours following exposure. A similar effect was observed in another study, and 1 week following exposure, tissue levels of 5HT were decreased by 25\% (Schmidt, 1987). When a smaller dose of 5.0 $\mathrm{mg} / \mathrm{kg}$ was administered, tissue levels of 5HT were also decreased within a 
few hours of exposure, but there was recovery 24 hours later in all brain regions examined (Stone et al, 1987).

In Wistar rats, $10.0 \mathrm{mg} / \mathrm{kg}$ MDMA produced relatively transient 5HT deficits in the frontal cortex (Garcia-Osta et al, 2004). Two hours following exposure, 5HT levels in the frontal cortex and hippocampus were decreased, with maximal effects produced 4-8 hours following exposure. 5HT levels in the frontal cortex recovered by 48 hours, but hippocampal levels of 5HT remained depressed for up to 7 days following exposure.

Both the (+) and (-) isomer decreased cortical 5HT by $80-90 \%, 3$ hours following exposure (10.0 mg/kg or $20.0 \mathrm{mg} / \mathrm{kg}$ MDMA), but only (+) MDMA produced a persistent and dose-dependent reduction in cortical 5HT (Schmidt, 1987). These findings suggest that there may be 2 different mechanisms underlying the earlier and later effects of MDMA on tissue levels of 5HT, possibly due to the effect of the 2 stereoisomers.

The effects of chronic exposure to MDMA on tissue levels of 5HT

Under some experimental conditions, a single dose of MDMA produced long-lasting deficits in tissue levels of 5HT, but multiple doses may be required to produce greater and more persistent deficits (Mokler et al, 1987). For example, a single injection of MDMA (40.0 mg/kg) produced persistent decreases of 5HT levels in the hippocampus, but not the frontal cortex, 16 days following exposure (Mokler et al, 1987). Four doses of MDMA (40.0 mg//kg administered every $2^{\text {nd }}$ day for 8 days), however, decreased 5 HT levels by over $50 \%$ in the frontal cortex, when measured 16 days after exposure. In another study, at least 2 injections of MDMA (10.0 $\mathrm{mg} / \mathrm{kg}$ ) were required to reduce 5HT levels in the frontal cortex (Battaglia et al, 1988). Multiple exposures of $8.0 \mathrm{mg} / \mathrm{kg}$ (weekly over 16 weeks) were also required to produces a deficit in tissue levels of 5HT that persisted for 10 weeks (Clemens et al, 2007). Similar results were observed in nonhuman primates; $5.0 \mathrm{mg} / \mathrm{kg}$ MDMA administered to squirrel monkeys decreased levels of 5HT in the hypothalamus and thalamus (Ricaurte et al, 
1988). When administered twice daily over 4 consecutive days, more widespread tissue 5HT deficits were produced (Ricaurte et al, 1988). Because multiple doses of MDMA administered over a short period of time reliably produced robust and persistent decreases in tissue levels of 5HT in non-human primates and rodents, the majority of animal studies have investigated long-term effects of the more stringent exposure regimens comprising 40.0-160.0 mg/kg MDMA (Battaglia et al, 1987; Battaglia et al, 1988; Commins et al, 1987; Lew et al, 1996; Sabol et al, 1996; O’Hearn et al, 1988; Sharkey et al, 1991).

The extent of 5HT deficits induced by multiple injections of MDMA is dose-dependent (Battaglia et al, 1998; Commins et al, 1987; Baumann et al, 2007; O'Shea et al, 1998). When measured 18 hours following MDMA exposure (twice daily injections of $5.0-20.0 \mathrm{mg} / \mathrm{kg}$ over 4 consecutive days), a dose of $5.0 \mathrm{mg} / \mathrm{kg}$ produced a $50 \%$ decreases in $5 \mathrm{HT}$ levels in the frontal cortex, but treatment with the higher dose $(10.0 \mathrm{mg} / \mathrm{kg} \& 20.0 \mathrm{mg} / \mathrm{kg})$ decreased tissue levels of 5 HT by over $80 \%$ (Battaglia et al, 1988). Dosedependent decreases in tissue levels of 5HT were also produced by a similar dosing regimen, when assays were conducted 2 weeks following exposure (Commins et al, 1987). When a less stringent dosing regimen of MDMA was used, 3 injections of $7.5 \mathrm{mg} / \mathrm{kg}$ every 2 hours produced a $50 \%$ decrease in $5 \mathrm{HT}$ but a lower dose $(1.5 \mathrm{mg} / \mathrm{kg}$ ) was ineffective (Baumann et al, 2007).

The frequency of MDMA administration can also influence the effect of MDMA on 5HT. For example, deficits in tissue levels of 5HT were not observed when $4.0 \mathrm{mg} / \mathrm{kg}$ was administered once daily for 4 days, or twice weekly for 8 weeks, but a reduction was produced following twice daily administration for 4 days (O'Shea et al, 1998). The results suggest that with low frequency dosing, there was recovery after each individual dose (O'Shea et al, 1998). MDMA also produced a dose- and frequencydependent reduction in cerebrospinal fluid levels of 5HT in rhesus monkeys (Insel et al, 1989).

The temporal parameters and the persistence of deficits have been investigated by measuring tissue levels of 5HT at various times following MDMA exposure (Scanzello et al, 1993; Sabol et al, 1996; Stone et al, 1987). In an extensive study, effects of multiple doses of MDMA (5 
injections of $5.0 \mathrm{mg} / \mathrm{kg}$ or $10.0 \mathrm{mg} / \mathrm{kg}$ over 24 hours) on tissue levels of 5HT were measured at various time points between 18 hours and 110 days (Stone et al, 1987). Magnitude and persistence of the deficit were dosedependent. Tissue levels of 5HT were reduced by $60-80 \%$ following administration of both doses in all brain regions examined. Thirty days following the low dose regimen, recovery was observed in all brain regions assayed except for the hippocampus, in which tissue levels of 5HT remained decreased even 110 days following exposure. Following administration of the high dose regimen, tissue levels of 5HT increased gradually over the 110 days, but a complete recovery was only observed in the hypothalamus. 5HT levels in the other brain regions examined remained reduced by about 40-50\%, even 110 days following exposure.

Two weeks following a dosing regimen consisting of 4 injections of $10.0 \mathrm{mg} / \mathrm{kg}$ MDMA (every 2 hours), regional brain levels of 5HT were markedly reduced (40\%-80\% depletions), and the rate of recovery was region-dependent (Scanzello et al, 1993). In the frontal cortex, there was a partial recovery 8 weeks later and full recovery was observed 16 weeks following exposure. The timecourse of recovery for striatal and hippocampal levels of 5HT was similar. The less persistent deficits observed in this study compared to the former study (Stone et al, 1987) could be due to the less stringent dosing regimen used.

Indeed, following a dosing regimen of twice daily injections of 20.0 mg/kg MDMA over 4 days, recovery was more gradual (Sabol et al, 1996). There was a $60-70 \%$ reduction in the frontal cortex, striatum and hippocampus, 2 weeks following exposure. Recovery occurred most rapidly in the striatum, and 5HT levels returned to normal 16 weeks following exposure. There was partial recovery of tissue levels of 5HT in the frontal cortex 16 weeks following exposure, and full recovery 52 weeks following exposure. Hippocampal 5HT levels, however, remained decreased even 52 weeks following exposure. These studies describe the robust and persistent effect of multiple injections of MDMA on tissue levels of 5HT, whereby the magnitude and persistence of effects are dose-, frequency- and regiondependent. 


\section{Neurotoxicity vs Neuroadaptation}

The pronounced effect of MDMA on 5HT neurotransmission in animals has prompted debate as to whether these effects occur as a result of loss of 5HT neurons and nerve terminals or is a neuroadaptive response to high levels of synaptic 5HT (Baumann et al, 2007; Biezonski \& Meyer, 2011).

\section{Neuronal degeneration}

Nerve terminal degeneration, quantified as silver staining, was observed in the striatum of rats that received 4 , but not 1 , injections of 80.0 $\mathrm{mg} / \mathrm{kg}$ MDMA (Commins et al, 1987). Four injections of $80.0 \mathrm{mg} / \mathrm{kg}$ MDMA also resulted in damaged cell bodies and nerve terminals in the somatosensory cortex; an effect that was less substantial following a single injection of $80.0 \mathrm{mg} / \mathrm{kg}$. Subsequent studies corroborated these findings and demonstrated dose-dependent neurodegeneration following high doses of MDMA (100.0 mg/kg- 600.0 mg/kg; Jensen et al, 1993).

There was neuronal damage in the frontoparietal cortex and the damage extended to deeper layers following administration of higher doses (Jensen et al, 1993). Silver-positive staining was also observed in the striatum and neocortex. Because 5HT cell bodies are not localised in the neocortex, this indicated that the neuronal damage was more generalised and included other systems. When measured 3 days following exposure to a single injection of MDMA (10.0-40.0 mg/kg), dose-dependent neuronal degeneration in the rat forebrain that extended to non-serotonin systems was also observed. Of interest, neuronal degeneration was only observed when the body temperature of rats exceeded $40.6^{\circ} \mathrm{C}$ (Schmued, 2003).

The role of hyperthermia in MDMA-produced neurotoxicity has been investigated in a number of studies, with equivocal results. Prevention 
of MDMA-induced hyperthermia attenuated 5HT deficits in some studies (Marlberg et al, 1996; Farfel \& Seiden, 1995; Colado et al, 1993; Goni-Allo et al, 2008) but not in other studies (Darvesh \& Gudelsky, 2004; O'Shea et al, 2006). Taken together, these findings suggest that MDMA may produce neurotoxicity that is dose- and temperature- dependent. This effect, however, is dissociable from MDMA-produced deficits in tissue levels of 5HT.

Another measure of neurotoxicity is axonal integrity (Callaghan et al, 2001; Kovacs et al, 2007). Anterograde axonal transport of MDMA pretreated rats was measured by tracing the transport of radioactive material injected into the dorsal raphe (Callahan et al, 2001). A decrease in this marker in multiple brain regions, similar to the profile of axotonomy induced by 5,7-DHT (a 5HT neurotoxin), was observed 3 weeks following the administration of MDMA (twice daily injection of $20.0 \mathrm{mg} / \mathrm{kg}$ over 4 days). While it is possible that this impairment reflects structural and functional damage, it could also be caused by a down regulation of cellular or metabolic processes which mediate this transport. Indeed, MDMA failed to alter fast axonal transport as determined by immunostaining of the amyloid precursor protein (APP; Kovacs et al, 2007).

Neurotoxicity can also be inferred by the presence of gliosis. MDMA, however, failed to activate microglial or astroglial processes (Pubill et al, 2003) or decrease INS and NAA levels in the striatum and frontal cortex (Perrine et al, 2010). When glial fibrillary acidic proteins (GFAP) were measured, gliosis was only observed following stringent dosing regimens of MDMA $(75.0 \mathrm{mg} / \mathrm{kg}$ or $150.0 \mathrm{mg} / \mathrm{kg}$ administered twice daily for 2 days; O'Callaghan \& Miller, 1993; Wang et al, 2004). The conversion of 5HTP-B to 5HT represents another means of assessing damage to 5HT neurons and following a 5HT-depleting regimen of MDMA, administration of 5HTP-B increased the 5HT immunoreactive signal in the brain, and restored 5HT levels to near control levels (Wang et al, 2007).

\section{SERT binding}


Density of SERT binding sites is a measure of pre-synaptic integrity and reductions in binding densities are generally interpreted as an indication of neurotoxicity (Fischer et al, 1995; Battaglia et al, 1988). Another possibility, however, is that the decrease in SERT binding indicates a loss of functional SERT expression. Therefore, interpretation of changes in SERTbinding must be made cautiously.

When measured over a 52 week period, MDMA decreased SERT binding densities, with partial recovery occurring between 18 hours and 10 weeks following MDMA exposure (Battaglia et al, 1988). Some deficits were still apparent 6 months following exposure, but by 12 months, there was full recovery (Battaglia et al, 1988). Two weeks following a less stringent dosing regimen (4 daily injections of $10 \mathrm{mg} / \mathrm{kg}$ ) MDMA reduced SERT (20-40\%) in all three brain regions examined (frontal cortex, striatum and hippocampus; Scanzello et al, 1993; Schenk et al, 2007; Fisher et al, 1995). When assays were conducted at various time points following exposure, SERT expression recovered by 32 weeks in the striatum and cortex, but deficits were still apparent in the hippocampus (Scanzello et al, 1993; Fisher et al, 1995). In rhesus monkeys, SERT binding densities was reduced 24 hours (Gould et al, 2011), but not 2 weeks (Banks et al, 2008) following the last self-administration session.

In order to confirm that MDMA-produced decrease in SERT expression was due to loss of nerve terminals, VMAT-2, a vesicular marker located in nerve terminals, was measured (Biezonski \& Meyer, 2010; Fantegrossi et al, 2004). MDMA failed to reduce VMAT-2 expression in rhesus monkeys, measured in vivo using PET imaging (Fantegrossi et al, 2004). In another study, MDMA decreased SERT, but not VMAT-2 protein levels (Biezonski \& Meyers, 2010), suggesting that 5HT nerve terminals were intact. Another possibility was that there was compensatory upregulation of VMAT-2.

Certainly, the effects of MDMA on SERT are different from effects produced by the selective neurotoxin, 5,7-DHT (Wang et al, 2005). As a result, it was hypothesised that MDMA induced internalization of SERT (Wang et al, 2005). If so, protein levels would not be expected to change even though binding densities would be decreased. Assays on SERT 
expression in subcellular fractions, however, revealed that MDMA did not alter the distribution of SERT between plasmalemma and endosomal compartments. It should be noted that the validity of these findings have been questioned due to questions about the specificity of the SERT antibody (Xie et al, 2006; Kivell et al, 2010). An in vitro study demonstrated internalization of SERT following MDMA exposure (Kittler et al, 2010). Live-cell confocal tracking also showed that MDMA treatment increased trafficking of SERT from the cell surface to cytoplasmic compartments in oocytes expressing SERT (Kivell et al, 2010). It will be important to determine whether this also occurs in vivo following MDMA exposure, but the findings suggest that following low doses of MDMA, neurotoxicity is not the major mechanisms for decreased 5HT. Rather, neuroadaptive mechanisms are more likely candidates.

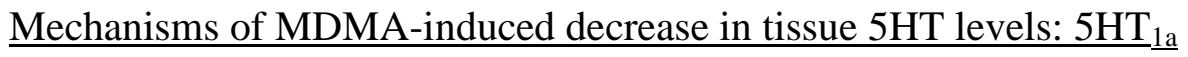
autoreceptor

If neuroadaptive mechanisms underlie the decreased 5HT neurotransmission, then it should be possible to facilitate recovery by targeting relevant mechanisms. Numerous studies have prevented MDMAinduced deficits (For example see, Malberg et al, 1996; Colado et al, 1999; Farfel \& Seiden, 1995; Rodsiri et al, 2010; Stone et al, 1988; Schmidt, 1987; Johnson et al, 1993), but there are few that have attempted to facilitate recovery following exposure.

Administration of selective 5HT reuptake inhibitors is one means of altering 5HT levels (SSRI; Blier, 2010). Following prolonged treatment with SSRIs, the $5 \mathrm{HT}_{1 \mathrm{a}}$ autoreceptor desensitizes, and 5HT synthesis and release are disinhibited (Stahl, 1999). Therefore, it might be possible to restore 5HT levels following MDMA exposure using chronic SSRI treatment. This idea was tested by administering the SSRI, fluoxetine (7.0 $\mathrm{mg} / \mathrm{kg} / \mathrm{day}$ for 37 days), 9-12 weeks following MDMA treatment (Thompson et al, 2004). Behavioural tests were conducted to assess social interaction, anxiety (emergency test) and depression-like behaviours (forced 
swim test; FST). Some of the depressive and anxiety symptoms produced by MDMA treatment were alleviated by fluoxetine, but deficits in measures of social interactions remained unchanged. It is noteworthy that the small decrease in tissue levels of 5HT was probably due to the prolonged abstinence period (14-17 weeks) between MDMA exposure and actual measurement. Chronic fluoxetine treatment did not restore tissue levels of 5HT in MDMA-treated rats or enhance them in control subjects. In another study, chronic fluoxetine treatment $(10.0 \mathrm{mg} / \mathrm{kg} / \mathrm{day}$ for 3 weeks $)$ altered behaviours in the FST and this was effect was attenuated by MDMA pretreatment (Durkin et al, 2008). This treatment, however, failed to reverse MDMA-produced decrease in tissue levels of 5HT (Durkin et al, 2008).

Despite the negative findings following fluoxetine administration, a number of studies have suggested that repeated exposure to many drugs of abuse (Cunningham et al., 1992; Kleven et al., 1995; Sastre-Coll et al., 2002; Kelaï et al, 2008; Esteban et al, 2002; Nevo et al, 1995) including MDMA (Renoir et al., 2008) results in a supersensitivity of the 5-HT $1 \mathrm{a}$ autoreceptor that was accompanied by reduced tissue levels of 5HT (Estaban et al, 2002; Renoir et al, 2008). In mice, a dosing regimen consisting of twice daily injections of $20.0 \mathrm{mg} / \mathrm{kg}$ MDMA over 4 consecutive days, decreased tissue levels of 5HT in the hippocampus, striatum and raphe nuclei (Renoir et al, 2008). MDMA exposure also increased sensitivity to 8-OH-DPAT-induced hypothermic response (an autoreceptor-mediated effect in mice), and decreased the spontaneous firing rate of dorsal raphe 5HT neurons. Furthermore, ipsapirone, a $5 \mathrm{HT}_{1 \mathrm{a}}$ receptor agonist, was more potent in inhibiting dorsal raphe 5HT neuron firing rates following MDMA exposure. Taken together, these findings suggest that MDMA exposure produced a supersensitivity of $5 \mathrm{HT}_{1 \mathrm{a}}$ autoreceptors and that this effect might underlie the decrease in tissue levels of 5HT.

The $5 \mathrm{HT}_{1 \mathrm{a}}$ autoreceptor is a Gi/o-protein coupled receptor (Blier et al, 1993), located on the cell bodies and dendrites of 5HT neurons in the raphe nuclei (Riad et al, 2000; Pazos \& Palacios, 1985). The $5 \mathrm{HT}_{1 \mathrm{a}}$ receptor is also located post-synaptically on non-5HT neurons (heteroceptors) and in regions innervated by 5HT neurons (Piñeryo \& Blier, 1999). In addition to differences in subcellular location, there are pharmacological differences 
between the heteroceptor and autoreceptor, with respect to G-protein coupling. The heteroceptor is coupled to the inhibition of adneylate cyclase activity (Chamberlain et al, 1993), but activation of the autoreceptor in the dorsal raphe produces downstream changes through inhibition of phospholipase C (Johnson et al, 1997; Clarke et al, 1996). It is under tonic activation from endogenous $5 \mathrm{HT}$, and regulates firing properties of $5 \mathrm{HT}$ neurons via coupling to an inward rectifying potassium channel (Katayama et al, 1997; Sprouse \& Aghajanian, 1987; Haddjer et al, 2004; Chaput et al, 1986).

Importantly, the $5 \mathrm{HT}_{1 \mathrm{a}}$ autoreceptor regulates $5 \mathrm{HT}$ synthesis (Hamon et al, 1988; Hjorth et al, 1982; 1996; Hjorth \& Magnusson, 1988; Bohmaker et al, 1993; Neckers et al, 1979). Low doses of the $5 \mathrm{HT}_{1 \mathrm{a}}$ selective agonist, 8-OH-DPAT, preferentially activates autoreceptors (Hjorth \& Magnisson, 1988) and dose-dependently decreased 5HT synthesis (Hjorth et al, 1982; Hjorth \& Magnusson, 1988). Intraraphe injection of the agonist, ipsapirone, or 8-OH-DPAT also inhibited 5HT synthesis, indicating a regulatory role of the $5 \mathrm{HT}_{1 \mathrm{a}}$ autoreceptor in synthesis (Hamon et al, 1988; Hjorth \& Magnusson, 1988).

It has been suggested that $5 \mathrm{HT}_{1 \mathrm{a}}$ autoreceptor activation modulates tryptophan hydroxylase (TPH; the rate-limiting enzyme for 5HT synthesis) activity (Boadle-Biber et al, 1993), providing a mechanism by which $5 \mathrm{HT}_{1 \mathrm{a}}$ autoreceptors regulate 5HT biosynthesis. For example, in rats, intra-raphe and systemic administration of gepirone, a $5 \mathrm{HT}_{1 \mathrm{a}}$ receptor agonist, blocked the increase in TPH activity produced by sound stress (Corley et al, 1992). In cell cultures, activation of $5 \mathrm{HT}_{1 \mathrm{a}}$ receptors decreased $\mathrm{TPH}$ mRNA expression (Wood \& Russo, 2001). Thus, 5HT biosynthesis might be autoregulated through $5 \mathrm{HT}_{1 \mathrm{a}}$ autoreceptor-mediated control of TPH gene transcription (Wood \& Russo, 2001).

Some studies have investigated the effect of MDMA exposure on 5$\mathrm{HT}_{1 \mathrm{a}}$ receptor binding characteristics (Aguirre et al., 1995; 1998; McGreggor et al., 2003). Twelve weeks following MDMA exposure, $5 \mathrm{HT}_{1 \mathrm{a}}$ heteroceptor binding was unchanged (McGregor et al, 2003). Attempts were made to determine differential changes in $5 \mathrm{HT}_{1 \mathrm{a}}$ autoreceptor and heteroceptor binding following MDMA exposure by comparing 8-OH- 
DPAT -binding in 5HT terminal regions and in the raphe nuclei (Aguirre et al, 1995; 1998). MDMA differentially altered $5 \mathrm{HT}_{1 \mathrm{a}}$ receptor expression across brain regions, and reduced binding in the raphe nuclei. These data suggested a desensitisation of $5 \mathrm{HT}_{1 \mathrm{a}}$ autoreceptors rather than supersensitivity, but specific alterations in autoreceptor sensitivity cannot unambiguously made exclusively on the basis of assays conducted on tissue from the cell body or terminal regions. $5 \mathrm{HT}_{1 \mathrm{a}}$ heteroceptors and autoreceptors are located in the raphe nuclei (Piñeryo \& Blier, 1999), and therefore simple binding assays cannot differentiate between the two.

Behavioural assays have also been developed to determine the response to $5 \mathrm{HT}_{1 \mathrm{a}}$ receptor agonists following chronic MDMA exposure (Granoff \& Ashby, 2001; McCreary et al, 1999). In rats, MDMA treatment ( $20.0 \mathrm{mg} / \mathrm{kg}$ daily for 4 consecutive days) produced an attenuation of the forepaw treading response to 8-OH-DPAT (Granoff \& Ashby, 2001). Other behavioural measures of $5 \mathrm{HT}_{1 \mathrm{a}}$ receptor sensitivity (8-OH-DPAT-induced flat body posture and hypothermia) were unchanged. Because all of the behavioural assays were related to post-synaptic $5 \mathrm{HT}_{1 \mathrm{a}}$ receptors (Granoff $\&$ Ashby, 2001), the effects on autoreceptor-specific responses were not determined

8-OH-DPAT-induced lower lip retraction (LLR) is one response that has been attributed to autoreceptor activation. It is produced by autoreceptor-selective (low) doses of the $5 \mathrm{HT}_{1 \mathrm{a}}$ receptor agonist, $8-\mathrm{OH}$ DPAT (Berendsen et al, 1991a; 1994; Li \& France, 2008; Koek et al, 1998; 2000) presumably by activation of autoreceptors located in the median raphe nucleus (Berendsen et al, 1994). Following chronic treatment with 8OH-DPAT that desensitized the autoreceptor (Kreiss \& Luki, 1992), there was a right-ward shift in the dose-response curve for 8-OH-DPAT-induced LLR (Berendsen et al, 1991). Further evidence for the LLR paradigm as a response of $5 \mathrm{HT}_{1 \mathrm{a}}$ autoreceptor activation comes from studies investigating ethanol withdrawal. An enhanced LLR response was found during abstinence from chronic ethanol exposure (Kleven et al, 1995), consistent with findings of $5 \mathrm{HT}_{1 \mathrm{a}}$ autoreceptor supersensitivity (Estaban et al, 2002; Nevo et al, 1995). 
In contrast, neurotoxic lesions produced by 5,7-DHT did not change the LLR response in rats (Berendsen et al, 1991), as might be expected. The failure to observe an effect of the lesion might be due to prior experience with other behavioural tests of effects of other 5HT agonists since there was an upward shift in the LLR response in vehicle-treated rats (Berendsen et al, 1991). Alternatively, compensatory mechanisms might have masked any effects of the lesion.

Mechanisms of MDMA-produced 5HT depletion: the role of tryptophan hydroxylase

A single and multiple exposures to MDMA decreased TPH activity (Stone et al, 1986; 1987; Schmidt \& Taylor, 1988). Three hours following a single injection of $10 \mathrm{mg} / \mathrm{kg}$ MDMA, TPH was reduced by $50 \%$ (Stone et al, 1986; Schmidt \& Taylor, 1988). Eighteen hours following multiple doses MDMA, TPH activity was reduced to $15-25 \%$ of controls (Stone et al, 1986). When assays were conducted for both TPH activity and tissue levels of $5 \mathrm{HT}$ at various time points (15 minutes-24 hours) following acute MDMA exposure $(10.0 \mathrm{mg} / \mathrm{kg})$, reductions in TPH activity preceded tissue 5HT deficits (Stone et al, 1987). Both TPH and 5HT recovered at the same rate and to the same extent over a 110 day period following chronic MDMA exposure (5 injections of $5.0 \mathrm{mg} / \mathrm{kg}$ or $10.0 \mathrm{mg} / \mathrm{kg}$; Stone et al, 1987).

TPH mRNA was reduced in frontal cortex 8 hours following MDMA exposure, but was increased by nearly 3 fold, 48 hours following exposure (Garcia-Osta et al, 2004). This increase in TPH mRNA expression was accompanied by a recovery of tissue levels of 5HT. In the hippocampus, TPH mRNA expression was reduced between 2 and 48 hours following exposure and was accompanied by decreased 5HT levels (Garcia-Osta et al, 2004). In the dorsal raphe, MDMA exposure (twice daily injections of 20.0 $\mathrm{mg} / \mathrm{kg}$ MDMA over 4 consecutive days) produced an increase in TPH mRNA expression, but TPH2 protein expression was decreased 2 weeks following exposure (Bonkale \& Austin, 2008). The decrease in TPH protein is consistent with previous reports of reduced TPH activity (Stone et al, 
1986; 1987; Schmidt \& Taylor, 1987; 1988), and the increase in TPH mRNA expression in the dorsal raphe and other brain regions might reflect a compensatory mechanism (Bonkale \& Austin, 2008; Garcia-Osta et al, 2004).

MDMA-produced alterations in TPH activity and biosynthesis are accompanied by a pronounced reduction in TPH immunoreactive fibres in Dark Agouti rats (Adori et al, 2010; 2011). It is noteworthy that Dark Agouti rats are particularly susceptible to the effects of MDMA as they are deficient in the CYP2D6 enzyme (Colado et al, 1995). The loss of TPH immunoreactivity persisted for up to 6 months in the hippocampus following a single exposure to MDMA (15.0 mg/kg; Adori et al, 2011). Three weeks following an intermittent schedule of MDMA exposure (4 doses of $15.0 \mathrm{mg} / \mathrm{kg}$ every $7^{\text {th }}$ day), there was a similar decrease (30-50\%) in TPH immunoreactivity, followed by recovery 3 months later (Adori et al, 2011). Six months following exposure, however, TPH immunoreactivity was above control levels in the parietal cortex and there was a trend towards an increase in other brain regions. It is possible that the faster rate of recovery of TPH immunoreactivity following an intermittent dosing regimen reflects a neuroprotective effect of prior experience (Adori et al, 2011; Bhide et al, 2009; Piper et al, 2010).

Studies consistently demonstrate that MDMA reduces TPH activity (Adori et al, 2010; 2011; Johnson et al, 1992; Schmidt et al, 1987; Schmidt \& Taylor, 1988; Stone et al, 1986; 1987; 1989; Bonkale \& Austin, 2008; Garcia-Osta et al, 2004), providing a mechanism of decreased 5HT synthesis. MDMA-produced decrease in TPH activity has been attributed to a reduction in the $\mathrm{V}_{\max }$ (Schmidt \& Taylor, 1987). Interestingly, in vitro experiments showed that MDMA had no effect on TPH activity in cortical slices or cortical synaptosomes (Schmidt \& Taylor, 1988), and did not interact with the protein directly (Schmidt \& Taylor 1987). Thus, the mechanism by which MDMA alters TPH activity is currently unknown. Nevertheless, it might be possible to reverse MDMA-produced 5HT deficits by increasing TPH activity. One procedure involved administration of the 5HT precursor, L-tryptophan (Esteban et al, 2010). 
A single dose of L-5HTP was effective in restoring tissue levels of 5HT following MDMA exposure (Wang et al, 2007). The persistence of this effect has not been determined but these initial findings suggest a potential means of permanently reversing effects. L-tryptophan and 5HTP, administered concomitantly with MDMA (twice daily injections over 4 days) prevented decreases in hippocampal 5HT and SERT binding (Sprague et al, 1994). Chronic L-tryptophan treatment increased tissue levels of 5HT by increasing TPH activity (Estaban et al, 2010).These studies raised the possibility that long-term treatment with $5 \mathrm{HT}$ precursors might be effective to reverse MDMA-produced deficits in tissue levels of 5HT. In order to adequately test this hypothesis, it is critical that MDMA exposure is consistent with the exposure following use and/or dependence.

The use of animal models of drug intake: dose \& "interspecies scaling"

One criticism of studies of effects of MDMA in laboratory animals is that doses that produce deficits are generally very high and might not reflect those consumed by users (Green et al, 2003; 2009; Baumann et al, 2007; Cole \& Sumnall, 2003). In a typical session users consume between 1-2 ecstasy pills (Winstock et al, 2001; Peroutka, 1987), consisting of 80150mg (Green et al, 2003). Based on these estimates, a 70kg person would consume approximately 1.0 to $4.0 \mathrm{mg} / \mathrm{kg}$, substantially less than the range of MDMA administered in a single dose in majority of animal studies (>10.0 $\mathrm{mg} / \mathrm{kg}$ ).

The question of how to compare doses of MDMA between humans and laboratory animals has been a topic of debate. Some researchers subscribe to the use of allometric scaling, based on body weight, size and other physiological variables to scale doses between humans and other species (Ricaurte et al, 2000). This technique is referred to as "interspecies scaling" and suggests that smaller animals require larger doses in order to achieve effects similar to humans (Green et al, 2003). Indeed, the substantially shorter elimination $\mathrm{T}_{1 / 2}$ of rats and non-human primates compared to humans would support this approach. In the case of 
“interspecies scaling”, a dose of 5mg/kg MDMA to a monkey produces effects comparable to a single dose of MDMA typically consumed by humans in a single session (Green et al, 2009; Schiffano et al, 2004).

The use of allometric scaling to produce comparable doses between rats and humans is not unequivocal. Because the metabolic pathway of MDMA differs between rats and humans, the use of an allometric equation, based on weight, body size and other physiological variables may not be feasible (De la Torre \& Farré, 2004; Baumann et al, 2007). On the other hand, it is important to note that the plasma concentration produced 45 minutes following a $10.0 \mathrm{mg} / \mathrm{kg}$ dose of MDMA in Dark Agouti rats (Colado et al, 1995) was comparable to that produced in humans following ingestion of a typical single dose of MDMA, i.e. 150mg tablet (Dowling et $\mathrm{al}, 1987)$. The $\mathrm{C}_{\max }$ in humans, following consumption of $2.0 \mathrm{mg} / \mathrm{kg}$, is only produced by administering $7.0 \mathrm{mg} / \mathrm{kg}$ in rats, producing a rat-human dose ratio comparable to that produced by interspecies scaling (Green et al, 2009).

Serotonin-reducing dosing regimens in laboratory animals may be comparable to multiple heavy binges of Ecstasy users (Parrott, 2001; Winstock et al, 2001). The exposure procedure, however, neglects the history of Ecstasy use that occurs in humans prior to heavy use. Thus, the doses administered in most animal studies may reflect heavy use, but they do not take into account the lengthy period of low and intermittent use that precedes these heavy levels of drug-taking (Parrott, 2001). The majority of the studies carried out in animal studies may not, therefore, accurately reflect effects of MDMA use. In contrast, self-administration of MDMA by laboratory animals may represent a more relevant method of drug exposure.

\section{$\underline{\text { Intravenous self-administration }}$}

The development of the chronic indwelling catheter (Weeks, 1962) was an important milestone in drug addiction research, as it allowed the evaluation of intravenous self-administration of drugs of abuse by laboratory animals. Self-administration has been demonstrated in a variety of laboratory animals, including non-human primates (Beardsley et al, 1986; 
Deneau et al, 1969; Lamb \& Griffiths, 1986; Goldberg et al, 1971), rats

(Schenk et al, 2003; Weeks \& Collins, 1964; Pickens \& Harris, 1968); mice (Grahame et al, 1995; Carney et al, 1991); cats (Kilbey \& Ellinwood, 1980; Balster et al, 1976) and dogs (Risner, 1975).

Drugs that are abused by humans are also reliably self-administered by laboratory animals (Schuster \& Thompson, 1969; Deneau et al, 1969) due to positively reinforcing properties. When presented with a choice between an active lever (which produces drug delivery upon depression), or an inactive lever (which produces no programmed consequence upon depression), a preference for the active lever was demonstrated (Griffiths et al, 1981; Pickens \& Harris, 1968). When the levers were reversed, there was corresponding reversal of lever pressing, again showing that the drug infusion was positively reinforcing (Pickens \& Harris, 1968). In addition, extinction of the behaviour upon substitution of the drug with vehicle, and subsequent reinstatement of the behaviour with reintroduction of the drug, convincingly demonstrated that operant responding was maintained by the reinforcing properties of the drug (Pickens \& Harris, 1968; Gerber \& Stretch, 1975; Grove \& Schuster, 1974; Hoffmeister \& Goldberg, 1973; Hoffmesiter et al, 1970).

A procedure that yokes drug infusions has also indicated drug reinforcement. In this procedure, there are triads of rats; subjects that selfadminister drug and subjects receiving non-contingent delivery of drug or vehicle according to responding by the response-contingent subject. Selfadministering subjects produced higher rates of responding compared to the yoked subjects, indicating that operant behaviour is not due to non-specific effects (Rasmussen \& Swedberg, 1998; Martello et al, 1995; Kuzmin \& Johansson, 2000; Donny et al, 1998; Blokhina et al, 2004). Vehicle-yoked controls show that responding reinforced by self-administration of drug infusions are not caused by environmental factors, such as handling, stress or exposure to the self-administration chamber (Sizemore et al, 2000; Cha et al, 1997).

The yoking procedure can also be employed to dissociate the purely pharmacological effects of drugs from the neuroplastic changes that are associated specifically with drug-taking behaviours. This procedure has 
revealed differences between effects of between contingent and noncontingent drug exposure (Reviewed by Jacobs, 2003) and has indicated that the context in which drugs are administered can have considerable influence (Edwards et al, 2007; Paladini et al, 2004; Orejarena et al, 2009; Stefanski et al, 1999; 2004; 2007; Frankel et al, 2007; Hanson et al, 2012; Metaxas et al, 2010; Rahman et al, 2004; Di Ciano et al, 1998; Smith et al, 1982; Crespo et al, 2001;2002;2003; You et al, 2007; 2008).

Self-administration is dose-dependent (Pickens \& Harris, 1968; Goldberg et al, 1971; Weeks \& Collins, 1964; Risner \& Goldberg, 1983; Wilson et al, 1971) and the relationship between number of operant responses and injection dose takes the shape of an inverted $\mathrm{U}$ (Yokel \& Pickens, 1973; Risner \& Goldberg, 1982; Schenk \& Partridge, 1997; Carnicella et al, 2011; Ator \& Griffiths, 1983; De Vry et al, 1989; Balster et al, 1976; Watkins et al, 1999). Low doses fail to support operant responding, but above threshold dose levels, the unit dose produces high levels of operant responding. As the dose of drug available increases further, responding decreases (Pickens et al, 1968; Goldberg et al, 1971). The relationship between dose and frequency of delivery has also been observed in humans who self-administered cocaine (Foltin \& Fischman, 1992; Dudish et al, 1996).

Response rates increase proportionally as the value of the FR schedule increases, so that the average daily intake of drug remains relatively constant (Pickens \& Harris, 1968; Yokel \& Pickens, 1973; Goldberg et al, 1971; Pickens, 1968). These findings suggest, at least during initial stages, responding changes in a compensatory manner to maintain a constant level of drug (Wilson et al, 1971).

Consistent with this idea, constant blood levels of drug were maintained, during self-administration of a range of cocaine and amphetamine doses (Yokel \& Pickens, 1974; Lau \& Sum, 2002) and a response was produced when the blood level of amphetamine fell below $0.2 \mu \mathrm{g} / \mathrm{ml}$ (Yokel \& Pickens, 1974). The behaviour is also determined by the neurochemical effects of the drug since responding was initiated when extracellular DA levels declined (Petitt \& Justice, 1991; Wise et al, 1995; Ranaldi et al, 1999). 
MDMA self-administration

There are a handful of laboratories that have demonstrated MDMA self-administration. In some studies, animals were trained to self-administer cocaine and MDMA reliably substituted (Beardsley et al, 1986; Lamb \& Griffiths, 1987; Fantegrossi et al, 2002; 2004; Banks et al, 2008; Lile et al, 2005) but other studies have produced MDMA self-administration in drugnaïve laboratory animals (Trigo et al, 2006; Ratzenboeck et al, 2001; Cornish et al, 2003; Feduccia et al, 2010; Reveron et al, 2006; 2010; Schenk et al, 2003; 2007, Daniela et al, 2004; 2006; Dalley et al, 2007; De la Garza et al, 2007).

In initial experiments with rhesus monkeys, MDMA substituted for cocaine, and inverted U-shaped functions for dose and responding were produced (Beardsley et al, 1986). Two doses of MDMA (30 $\mu \mathrm{g} / \mathrm{kg} / \mathrm{infusion}$ $\& 100 \mu \mathrm{g} / \mathrm{kg} /$ infusion) maintained responding above saline levels for 2 out of 4 monkeys, but 1 subject did not self-administer MDMA, regardless of the dose (Beardsley et al, 1986). On the other hand, 2 of the subjects responded at higher levels than when cocaine was self-administered, when the $100 \mu \mathrm{g} / \mathrm{kg} /$ infusion dose of MDMA was available. In baboons, there was high variability both within subjects and across sessions and overall responding was relatively low (5-6 responses produced by a $1.0 \mathrm{mg} / \mathrm{kg} /$ infusion dose; Lamb \& Griffiths, 1987). Subsequent studies in rhesus monkeys showed that racemic MDMA and both the (+) and (-) isomers (0.003-0.3 mg/kg/infusion) reliably substituted for cocaine, and inverted U-shaped dose-response curves were produced (Fantegrossi et al, 2002; 2004). Overall responding maintained by MDMA was about $60 \%$ (Fantegrossi et al, 2002) and 80\% (Fantegrossi et al, 2004) of responding maintained by cocaine, suggesting that MDMA is a less efficacious reinforcer.

Similar results have been produced in rat subjects, In food or sucrose-trained rats, overall responding maintained by MDMA was low (Ratzenboeck et al, 2001; De la Garza et al, 2007). Inverted U-shape 
functions for dose and responding were produced, but only 1-4 responses were produced during 2 hour sessions (Ratzenboeck et al, 2001). In another study, responding maintained by MDMA was not different from responding maintained by saline (De la Garza et al, 2007). Other studies in rats also failed to demonstrate high rates of operant responding (Reveron et al, 2006; 2010; Fedducia et al, 2010; Cornish et al, 2003).

Some studies have, however, produced higher rates of MDMA selfadministration (Schenk et al, 2003; 2007; Daniela et al, 2004; 2006; Dalley et al, 2007). Indeed, experimental parameters such as infusion time, session length and time-out duration may have considerable influence on the propensity for laboratory animals to self-administer MDMA (De la Garza et al, 2007).

Dose of MDMA can also impact acquisition of MDMA (Schenk et al, 2003). When a 1.0mg/kg dose was available, 15-20 responses were made, and when the dose was decreased to $0.5 \mathrm{mg} / \mathrm{kg}$, overall responding increased in a compensatory manner to about 40-60 responses (Schenk et al, 2003). Additionally, unlike self-administration of many other drugs, MDMA selfadministration proceeds with a long latency and intake escalates over days (Schenk et al, 2003; 2007). Following acquisition, responding increased as the FR value increased (Daniela et al, 2006) and when saline was substituted for MDMA, behaviour was extinguished, and was subsequently reinstated when MDMA became available again (Schenk et al, 2003; Daniela et al, 2006).

The pattern of MDMA self-administration compares favourably to human drug-taking. Rats initially self-administer relatively low doses of 1.0 $\mathrm{mg} / \mathrm{kg}$ to $2.0 \mathrm{mg} / \mathrm{kg}$ per session (Schenk et al, 2003; 2007). With continued testing, intake escalates for some and approximately $50-60 \%$ of the original cohort acquired high rates of self-administration (Schenk et al, 2003; 2007). It has been suggested that this reflects changes in drug-taking patterns that would occur in humans who consume large quantities of MDMA (Schenk, 2009; 2011). 


\section{Effects of drug self-administration on NOR}

Self-administered methamphetamine and cocaine produced deficits in NOR that were more prominent when long access sessions were used (Reichel et al, 2011; Rogers et al, 2008; Brians et al, 2008). In one study, NOR testing was conducted 7 days after methamphetamine selfadministration (7 daily 1-hour sessions \& 14 daily 6-hour sessions). Deficits in NOR were revealed in methamphetamine self-administering rats, following 90 minute and 24 hour ITIs (Riechel et al, 2011). In another study, NOR deficits were observed 10-12 days after long (6 hours)-, but not short (1 or 2 hours)-access sessions of methamphetamine self-administration (Rogers et al, 2008). Following cocaine self-administration, NOR decayed more rapidly as a function of ITI in a long-access group compared to a short-access group (Briand et al, 2008). Following an ITI of 3 hours, recognition indexes (\% time spent with novel object) were similar in control and cocaine-treated rats. After a 24 hour ITI, the long access cocaine-selfadministration group had a lower recognition index than that of the shortaccess cocaine self-administration group and controls, suggesting disrupted NOR. Following an ITI of 1 week, deficits in both cocaine selfadministering groups were observed.

Following cocaine self-administration, ITI-dependent deficits in NOR suggested impaired memory (Briand et al, 2008), but NOR deficits observed in methamphetamine self-administration studies may be underpinned by deficits in other cognitive processes (Rogers et al, 2008; Riechel et al, 2011). These studies also demonstrated that long access sessions of self-administration produced more pronounced deficits than short-access sessions (Briand et al, 2008; Rogers et al, 2008). Long access sessions are characterised by escalation of drug intake that is consistent with dependence and therefore, disrupted NOR might be restricted to subjects that are dependent. It is also possible, however, that it is a dose-dependent effect.

In these studies, intact NOR following relatively long ITIs (24 hours -1 week) would indicate long-term memory, rather than working memory 
processes (Briand et al, 2008; Riechel et al, 2011), which typically decays over a matter of minutes, rather than days (Ricaurte et al, 1993; Marston et al, 1999). Nevertheless, these studies raise the possibility that selfadministration of drugs of abuse produce deficits in NOR. Evidence suggests that MDMA exposure produces deficits in NOR, but there is no information regarding the effect of self-administered MDMA on NOR.

\section{MDMA self-administration and 5HT}

The decrease in tissue levels of 5HT is perhaps one of the most welldocumented, reliable, and prominent outcome of MDMA exposure. There has, however, been limited exploration into the effects of MDMA selfadministration on the 5HT system in laboratory animals. In contrast to effects of experimenter-administered MDMA, self-administration by monkeys failed to decrease 5HT (Fantegrossi et al, 2004). It is possible that the failure to detect decreases in tissue levels of 5HT resulted from a low daily dose of MDMA that was self-administered (1-3mg/kg), and a long abstinence period (2 months) between exposure and assays. MDMA selfadministration also failed to alter SERT binding densities in non-human primates, when measured 24 hours following the last session of selfadministration (Banks et al, 2008). These primates, however, all had a prior history of cocaine self-administration that may have masked the effects of MDMA (Banks et al, 2008). Following self-administration of MDMA in rats, however, SERT binding densities were reduced 2 weeks following exposure (Schenk et al, 2007). There was also a 30\% decrease in tissue levels of 5HT, 5 days following MDMA self-administration $(165.0 \mathrm{mg} / \mathrm{kg}$; Schenk et al, 2010). These studies show that high levels of self-administered MDMA produce alterations in 5HT, but there is little information regarding the parameters of this effect. 


\section{The Present study}

The human literature suggests persistent brain and behavioural abnormalities following heavy consumption of Ecstasy. Because of many confounds, it is difficult to attribute these abnormalities specifically to MDMA exposure. Animal studies provide a more controlled means of measuring chronic effects of MDM, but results have been questioned primarily due to the dosing regimens generally administered. Certainly, it is difficult to determine doses in animals that compare to human usage, in part, due to differences in pharmacokinetics and metabolism of MDMA across species. In addition, dosing schedules do not generally emulate the pattern of drug-taking in humans. Self-administration provides a means of tracking effects of MDMA during acquisition and maintenance and may therefore more closely mirror patterns of consumption. The current thesis aims to determine the effect of MDMA self-administration on cognition, assessed by performance in NOR paradigm, and on tissue levels of 5HT. Response of the $5 \mathrm{HT}_{1 \mathrm{a}}$ autoreceptor sensitivity will be measured using the LLR paradigm and the ability of 3 pharmacological treatments to reverse MDMA-produced 5HT deficits will be determined. 


\section{General Methods}

\section{$\underline{\text { Subjects }}$}

Male Sprague-Dawley rats weighing 300-350g (self-administration experiments) or 220-250g (for experimenter-administered experiments) at the start of the study were bred in the vivarium at Victoria University of Wellington, New Zealand. The rats were housed in groups of three or four until testing, after which they were housed singly in standard polycarbonate hanging cages. Food and water were available ad libitum at all times except during testing. The rats were housed in a temperature $\left(21^{\circ} \mathrm{C}\right)$ and humidity $(55 \%)$ controlled colony maintained on a $12 \mathrm{~h}$ light/dark cycle with lights on at 07:00.

\section{$\underline{\text { MDMA self-administration }}$}

Surgery

Surgical procedures were carried out on a warming pad and eyes were coated with Vaseline to prevent drying out. Silastic catheters were implanted in the external jugular vein under deep anaesthesia induced by separate i.p. injections of ketamine $(90.0 \mathrm{mg} / \mathrm{kg}$, i.p) and xylazine (5.0 $\mathrm{mg} / \mathrm{kg}$, i.p). The external jugular vein was isolated from surrounding tissue and tied off. A small incision was made in the vein through which the 
catheter was inserted and fixed in place with super glue and suture thread. The distal end of the catheter was then passed subcutaneously to an exposed section of the skull. The distal end of the tubing, fitted onto a $2 \mathrm{~cm}$ length of 22-guage stainless steel tubing, was secured via four jeweller's screws embedded in dental acrylic which was cemented onto the skull. Selfadministration testing commenced 7 days following surgery.

Post-operation care included s.c. administration of the antiinflammatory Carprofen $(5.0 \mathrm{mg} / \mathrm{kg})$ and electrolytes $(10.0 \mathrm{ml} 0.9 \% \mathrm{NaCl}$, s.c.) were administered. Daily flushing of catheters with a sterile $0.9 \%$ saline/heparin (30 UI/ml) solution which contained penicillin $\mathrm{G}$ potassium $(250,000 \mathrm{UI} / \mathrm{ml})$ helped to maintain catheter patency and prevent infection. Carprofen $(5.0 \mathrm{mg} / \mathrm{kg})$ was administered again at 24 hours and 48 hours following surgery.

Apparatus

Self-administration was conducted in standard operant chambers (Med Associates, ENV-001) equipped with two levers; an active lever and an inactive lever. Depression of the active lever resulted in a 12.0 second intravenous infusion of MDMA $(0.1 \mathrm{ml})$ synchronized with the illumination of a stimulus light located above the lever. Inactive lever responses were recorded but had no programmed outcome.

A $20.0 \mathrm{ml}$ syringe was housed in a mechanical pump (Razel with 1 rpm motor) and connected to a length of microbore tubing through a swivel. The length of tubing was attached to the exposed stainless steel end of the catheter during testing. A microcomputer interfaced with Med Associates software controlled drug delivery and data acquisition.

Procedure

MDMA self-administration sessions were $2 \mathrm{hr}$ duration 6 days a week and were conducted between 0700 and 1900 hours. Catheters were 
flushed with $0.2 \mathrm{ml}$ of the penicillin-heparin solution prior to and immediately after each session. Each session began with an experimenterdelivered infusion of drug. Subsequently, each infusion was delivered automatically following depression of the active lever according to a fixed ratio 1 reinforcement schedule.

A dose of $1.0 \mathrm{mg} / \mathrm{kg} /$ infusion served as the reinforcer until a total of 90 infusions was self-administered. Rats that failed to self-administer these initial 90 infusions within 30 test days were considered to have not acquired self-administration and further testing was not conducted. For the remaining rats, the dose was decreased to $0.5 \mathrm{mg} / \mathrm{kg} /$ infusion once this initial criterion had been met. Two different criteria for total MDMA intake were used. For the low intake criterion, self-administration continued until an additional 150 infusions $(75 \mathrm{mg} / \mathrm{kg})$ were self-administered. For the high intake criterion, self-administration continued until an additional $450(225 \mathrm{mg} / \mathrm{kg})$ infusions were self-administered. Control rats were placed in the test chambers during 2 hour sessions, 6 days per week for 4 weeks, but did not receive any MDMA.

\section{$\underline{\text { MDMA Pre-treatment }}$}

MDMA (10.0 mg/kg in $0.9 \%$ saline vehicle; $1.0 \mathrm{mg} / \mathrm{ml}$ i.p.) was administered once every 2 hours, for a total of 4 injections. This dosing regimen was chosen as it had been previously shown to produce decreases in tissue 5HT levels in the frontal cortex, hippocampus and striatum (Scanzello et al, 1993), 2 weeks following exposure. 


\section{Novel Object Recognition}

NOR apparatus and materials

NOR testing began 7 or 70 days following the last selfadministration session. Tests were conducted in a square chamber with transparent Pyrex walls $(42 \mathrm{cmX} 42 \mathrm{cmX} 30 \mathrm{~cm})$ enclosed in a sound-proof cupboard. The chamber and objects (see Table 1 for object characteristics) were thoroughly cleaned with Virkon detergent between each test. Fourteen different pairs of objects were used. Objects were chosen to have features that were thought to be distinguishable to rats but not bear any natural significance or be associated with any reinforcement. The objects chosen were made of plastic, glass, ceramic or metal to minimize retention of olfactory cues. Object orientation remained constant throughout testing. Objects were secured in the box with blue tack, making sure no blue tack was exposed. Behaviour during T1 and T2 was recorded on video using a camera that was positioned overhead 


\begin{tabular}{|l|l|}
\hline Object & Description \\
\hline Pink hole puncher & A metal hole puncher with round edges \\
\hline Tape dispenser & $\begin{array}{l}\text { A blue plastic tape dispenser with round edges and } \\
\text { sharp parts removed }\end{array}$ \\
\hline Plastic bottle & A clear plastic bottle 700ml \\
\hline Beer bottle & A green glass bottle with long narrow neck \\
\hline Can & A 355ml metal can \\
\hline Tea cup & A short red and white ceramic cup with handle \\
\hline Green hole puncher & $\begin{array}{l}\text { A green metal hole puncher, pressed down with } \\
\text { square edges }\end{array}$ \\
\hline Mug & A tall blue ceramic mug with handle \\
\hline Beaker & A tall glass beaker \\
\hline Glass & A short round glass \\
\hline Funnel & A plastic funnel placed upside down during testing \\
\hline Tuna can & A metal tuna can with no label \\
\hline Glass bottle & A tall bottle made of glass \\
\hline Egg cup & A small white ceramic egg cup \\
\hline
\end{tabular}

Table 1. Objects used in the NOR task

\section{Procedure}

Prior to testing, rats were habituated to the testing apparatus with two exploration sessions without objects ( 5 minutes each). Tests began $24 \mathrm{~h}$ following the last habituation period. Seven daily sessions of NOR were conducted during a 1-week period. Each session consisted of two trials of 3 minutes each (T1 and T2) in which rats were allowed to explore two objects in the chamber. During T1, identical objects (sample and duplicate) were placed at the far side of the box, equidistant from the walls. Rats were placed in the box facing away from the objects and allowed to explore freely and were then removed from the box. After a variable ITI of 1, 10, 15, $20,25,30$ or $60 \mathrm{~min}$, rats were returned to the box where a different sample had been placed and the duplicate had been replaced with a novel object. 
All rats received tests following all ITIs and the order of tests of the different intervals was counterbalanced across the group. The position of the novel object (east vs. west) was also counter-balanced across sessions and rats. A new pair of sample objects and novel object was used for each ITI so that no rat was exposed to the same object twice across sessions. Objects were randomly assigned to sessions.

\section{Exploratory behaviour scoring and analysis}

Exploration of objects was considered when the rat's nose was within $1 \mathrm{~cm}$ of the object, oriented towards it. Time spent exploring during T1 and T2 was determined. Similarly, an approach towards the object was counted as the directing of the nose towards and within $1 \mathrm{~cm}$ of the object. If the rat approached the object and then turned away for a period of time without moving the hind legs but then oriented towards the object again, two approaches were scored. Climbing on objects or rearing was not considered to be exploratory behaviour if the previous stipulations were not met. A criterion of at least $20 \mathrm{~s}$ exploration time for both objects combined in T1 was imposed.

\section{$\underline{\text { Lower Lip Retraction }}$}

During testing, animals were individually caged in transparent polycarbonate cages, in a separate testing room. A full lower lip retraction was scored when the full length of the lower incisors could be seen and the lower lip was clearly retracted. A partial LLR was scored when more than normal of the lower incisors could be seen but the full length was not exposed. A 'no LLR' was scored when the lower incisors could not be seen at all or if only the tip of the incisors could be seen. A full lower lip retraction was assigned a score of 2 , a partial 1 and a 'no' 0 , so that a maximum score of 12 could be scored for each subject.

The LLR is a continuous behaviour and was scored by its partial or full presence by an experimenter every 10 minutes (for 60 minutes), 
following a challenge dose of 8-OH-DPAT. 8-OH-DPAT was administered via s.c. injection with increasing doses every hour according to the following schedule: $0.0 \mathrm{mg} / \mathrm{kg}, 0.003 \mathrm{mg} / \mathrm{kg}, 0.01 \mathrm{mg} / \mathrm{kg}, 0.03 \mathrm{mg} / \mathrm{kg}$ and finally $0.1 \mathrm{mg} / \mathrm{kg}$. One experimenter was responsible for observing the behaviour and thus scoring each subject a 0,1 or 2 . This experimenter was blind to the experimental group of each subject as well as the previous scores of the subject, to prevent any bias that might occur if the experimenter scoring the behaviour was able to see the score of the previous 10 minutes. Another experimenter was responsible for administration of the challenge dose of 8-OH-DPAT and recording the subject's scores over the course of LLR testing.

\section{Neurochemical Assay}

\section{Tissue extraction}

Tissue extraction was carried out either 2 or 10 weeks following the completion of self-administration for both the low and high intake group, or 2 weeks following experimenter-administered MDMA. Rats were rendered unconscious by $\mathrm{CO}_{2}$ asphyxiation before decapitation and their brains were rapidly removed. The frontal cortex, striatum and hippocampus were dissected on ice using a brain block (Heffner et al, 1980). These regions were chosen as they are richly innervated with 5HT and previous studies have shown decreased tissue levels following experimenter-administered MDMA. Tissue samples were weighed and stored at $-80^{\circ} \mathrm{C}$ until further analyses were conducted for the measurement of 5HT.

\section{Tissue preparation and HPLC analysis}

Tissue samples were combined with $10 \mu 10.1 \mathrm{~N}$ perchloric acid per $1 \mathrm{mg}$ tissue, homogenized and centrifuged (13,000 rpm) for 30 minutes at $4^{\circ} \mathrm{C}$. The supernatant of each sample was then filtered into vials. Samples 
were injected into a high performance liquid chromatography system with electrochemical detection equipped with a C18 reversed phase column (Agilent Eclipse XDB-C18, 150×4.6 mm, $5 \mu \mathrm{m}$ particle size) and a coulometric detector (Coulochem III, ESA Inc.; analysis cell, -175 and +400 mV; guard cell, $+450 \mathrm{mV}$ ). The mobile phase ( $75 \mathrm{mM}$ sodium dihydrogen phosphate, $1.7 \mathrm{mM}$ octanesulphonic acid, $0.25 \mathrm{mM}$ disodium EDTA, $100 \mu 1 / 1$ triethylamine, $10 \%(\mathrm{v} / \mathrm{v})$ acetonitrile, $\mathrm{pH}$ 3.0) was delivered at the constant flow rate of $1.0 \mathrm{ml} / \mathrm{min}$. The height of the $5 \mathrm{HT}$ peaks of samples and calibration standards were obtained and the concentration of 5HT in tissue samples was then calculated using the regression equations obtained from the calibration standards. The range of standards used was $15.125 \mathrm{ng} / \mathrm{ml}-62.5 \mathrm{ng} / \mathrm{ml}$ for the frontal cortex, $31.25 \mathrm{ng} / \mathrm{ml}-125 \mathrm{ng} / \mathrm{ml}$ for the hippocampus and $125 \mathrm{ng} / \mathrm{ml}-500 \mathrm{ng} / \mathrm{ml}$ for the striatum. The lower limit of detection of $5 \mathrm{HT}$ was $0.5 \mathrm{ng} / \mathrm{ml}$ in the frontal cortex and hippocampus and $0.2 \mathrm{ng} / \mathrm{ml}$ in the striatum. 
$\operatorname{MDMASA}($ low $) \stackrel{1 \text { week }}{10 \text { weeks }} \longrightarrow \begin{gathered}\text { NOR } \\ (1 \text { week })\end{gathered}$

MDMA SA (low/high) $\underset{2 \text { weeks }}{10 \text { weeks }} 5 \mathrm{HT}$

MDMA SA (high) $\longrightarrow$ weeks $\longrightarrow$ LLR

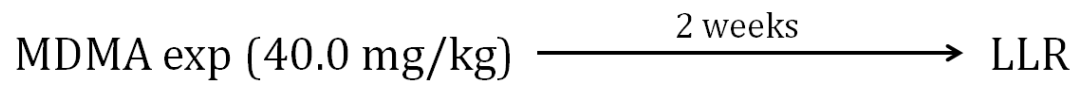

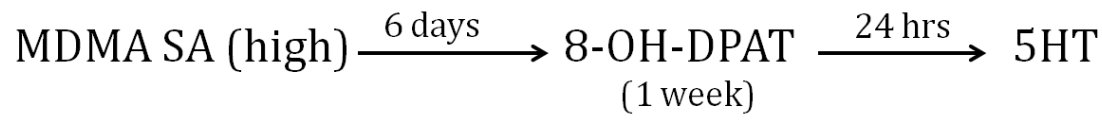

MDMA exp $(40.0 \mathrm{mg} / \mathrm{kg}) \stackrel{2 \text { days }}{\longrightarrow} \underset{(2 \text { weeks })}{\operatorname{Trazadone}} \longrightarrow 5 \mathrm{HT}$

MDMA exp $(40.0 \mathrm{mg} / \mathrm{kg}) \stackrel{6 \text { days }}{\longrightarrow}$ Tryptophan $\stackrel{24 \mathrm{hrs}}{\longrightarrow} 5 \mathrm{HT}$ (1 week)

Figure 2.1 Timelines describing experimental protocols

\section{$\underline{\text { Drugs }}$}

- +/- 3,4- Methylenedioxymethamphetamine hydrochloride (MDMA, ESR, Porirua, New Zealand), dissolved in a solution of $0.9 \%$ saline

- ( \pm )-8-Hydroxy-2-dipropylaminotetraline hydrobromide (8-OHDPAT; Tocris), dissolved in $0.9 \%$ saline

- Trazodone hydrochloride (Trazodone; Sigma-Aldrich), dissolved in $50 \%$ DMSO

- L-Tryptophan (tryptophan; Sigma-Aldrich), dissolved in $0.9 \%$ saline

I.v. infusions were in a volume of $0.1 \mathrm{ml} / \mathrm{kg}$ and i.p. and s.c. injections were in a volume of $1.0 \mathrm{ml} / \mathrm{kg}$. All drug doses refer to the salt weight unless otherwise stated (base weight; b.w.). 


\section{Statistics}

For NOR, differences in absolute time spent exploring the two objects during $\mathrm{T} 1$ and $\mathrm{T} 2$ were analysed using repeated dependent sample ttests. A recognition index was calculated as ((time with novel/(time with familiar + time with novel)). A proportion of 0.5 indicates equal time spent with the two objects. These data were analysed from T1 as well as T2 using analysis of variance (ANOVA) to determine changes as a function of ITI.

For neurochemistry, separate two-way ANOVAs were conducted on tissue levels of 5HT for each of the three brain sites using two factors:

MDMA intake (high versus low) X abstinence (2 weeks versus 10 weeks; Chapter 5) or MDMA pre-treatment X post-treatment (Chapter $6 \& 7$ ).

All analyses were conducted using SPSS statistical packages (SPSS Inc; version 18.0 for Windows 2007). 


\section{Chapter 3: Preliminary experiments; Establishing the NOR task using short inter-trial intervals in drug naïve-rats}

(These findings have been published in Schenk S., Harper D. \& Do (2010) Novel object recognition memory: measurement issues and effects of MDMA self-administration following short inter-trial intervals. Journal of Psychopharmacology, 25: 1043-1052. Do's contribution was data collection, data analysis and some of the manuscript writing)

\section{Overview}

For the recognition index to be validated as a measure of memory for a familiar object there must be decay, representative of a 'forgetting function', as the ITI is increased. Establishing a 'forgetting function' that depicts the decay of object recognition memory over time is, therefore, an integral aspect of validating the task as a true measurement of memory. Unfortunately, numerous studies which make use of this task only test performance following a single, usually long, ITI (For example see Winnicka et al, 1997; Giovannini et al, 1999; Lieben et al, 2004; 2005; Puma et al, 1999; Scali et al, 1997; Lebrun et al, 2000). Furthermore, there appears to be no general consensus across these studies as to which ITI is most suitable to test NOR, making comparisons across studies difficult. The purpose of these preliminary experiments was to test a range of ITIs in order to establish a forgetting curve using the NOR task in drug naïve rats $(n=15)$.

$\underline{\text { Results }}$

In order to establish the forgetting curve for NOR, both the exploration time and number of approaches were measured as dependent variables. Figure 3.1 shows the absolute time (in seconds) spent exploring objects (top) and the proportion of time spent exploring the duplicate (to be novel) object (bottom) during T1 as a function of groups. Total exploration in T1 was 30-40 s. There was no significant difference in overall time spent 
exploring the two objects ( $p>0.05$ for all seven comparisons) suggesting a lack of overall position bias. The proportion of time during $\mathrm{T} 1$ spent with the duplicate object that was replaced in T2 with a novel object, however, shows a range, from 0.43 to 0.55 . The largest positive bias occurred in the 20 min ITI group.
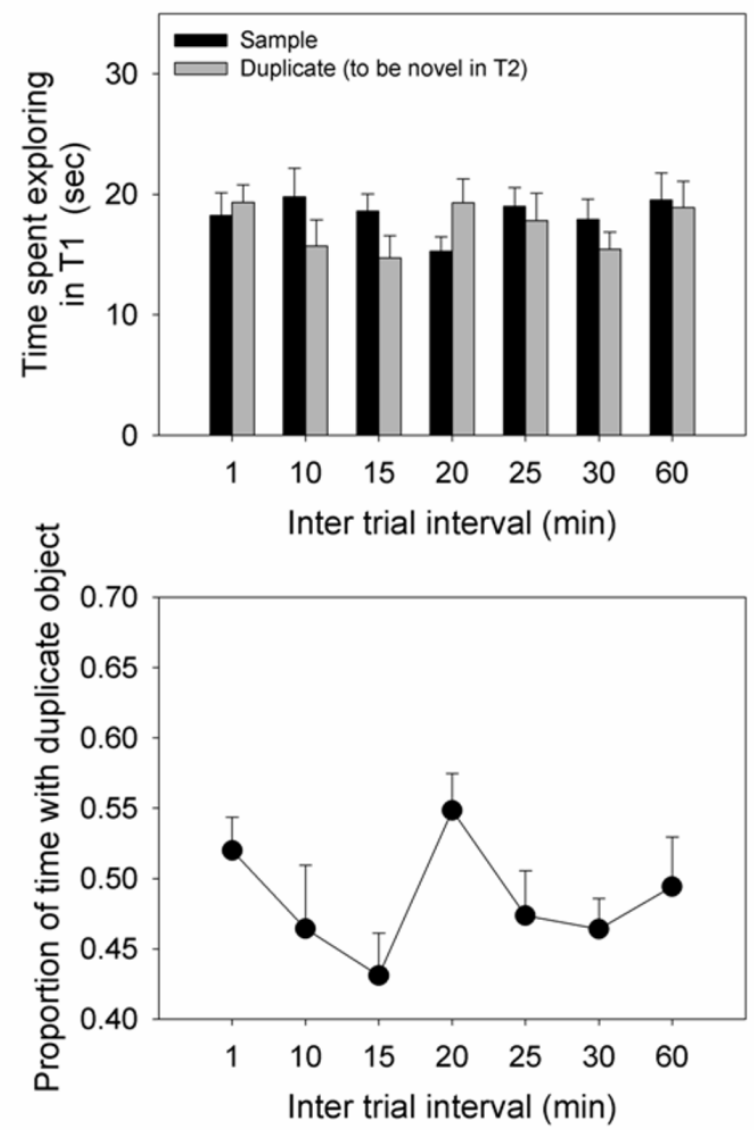

Figure 3.1 Time (in seconds) spent exploring objects (top) and the proportion of time spent exploring the duplicate (to be novel) object (bottom) during T1 as a function of ITI. Bars represent mean time (top) or mean proportion of time (bottom) (+SEM) 
Figure 3.2 shows the data for exploration (top) and proportion of time with the novel object (bottom) during T2. Absolute exploration times were also around $40 \mathrm{~s}$, but the novel object was explored more than the familiar object following some of the ITIs. ANOVA revealed a significant effect of object (familiar versus novel; $F(1,223)=16.429$, $p<0.05$ ), ITI $(\mathrm{F}(6,223)=2.77, \mathrm{p}<0.05)$, but no interaction $(\mathrm{F}(6,223)=1.11, \mathrm{~N} . \mathrm{S})$.

Following the shortest $(1 \mathrm{~min})$ and longest interval tested $(60 \mathrm{~min})$ about 0.6 of exploration time during the 3 min session was devoted to exploration of the novel object. Following the intermediate ITIs, proportion of time spent with the novel object ranged from 0.51 to 0.54 , overlapping considerably with the data from T1. Thus, there was no apparent relationship between ITI and NOR and a decay curve as a function of ITI was not produced. 

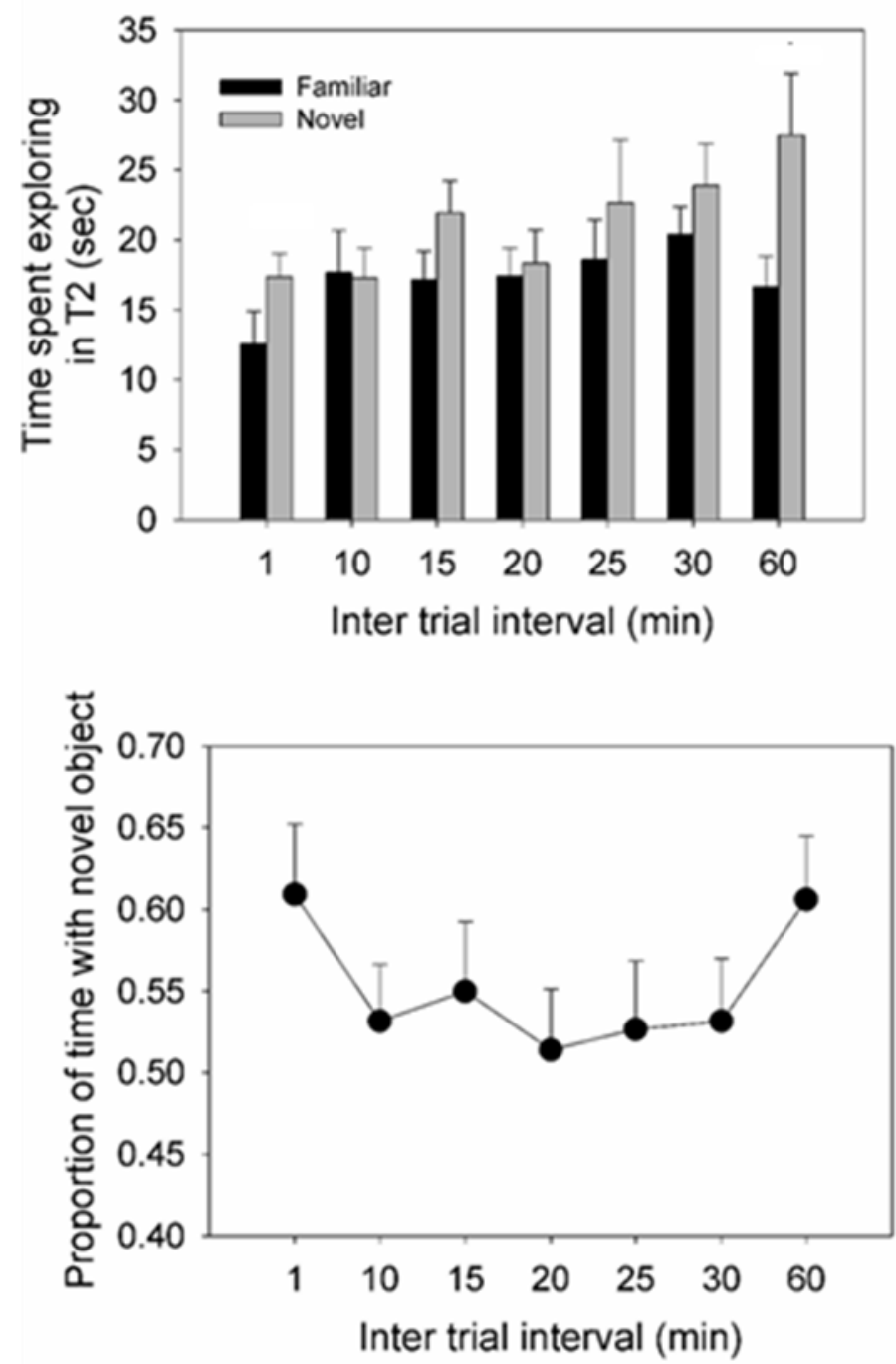

Figure 3.2 Time (in seconds) spent exploring objects (top) and the proportion of time spent exploring the duplicate (to be novel) object (bottom) during T2 as a function of inter-trial interval. Bars represent mean time (top) or mean proportion of time (bottom) (+SEM).

Figure 3.3 directly compares the data from $\mathrm{T} 1$ and $\mathrm{T} 2$ and shows the proportion of time spent with the duplicate (T1) and novel (T2) objects as a function of ITI. Thus, these data take into consideration any initial position bias when identical objects were presented during T1 that might impact the data. With the exception of the 20 min ITI, there was an increase in the proportion of time spent with the novel object and ANOVA revealed a significant effect of test day $(F(1,81)=13.37, p<0.01)$ but no interaction 
$(\mathrm{F}(6,81)=1.848, \mathrm{NS})$. The differences ranged from -0.03 to 0.11 but were not an obvious function of ITI.

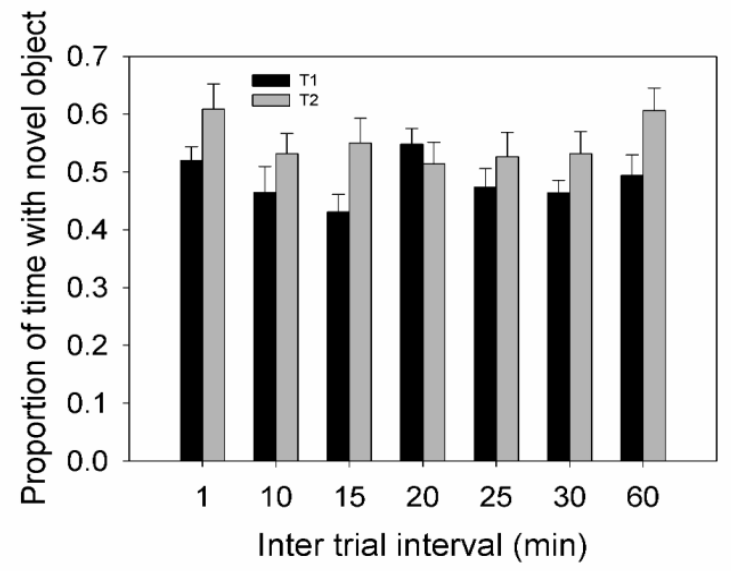

Figure 3.3 Mean proportion of time (+SEM) spent with the duplicate (in T1) and novel (in T2) object as a function of inter-trial interval.

It is possible that the 'novelty' of the novel object was limited to a short period during T2 and dissipated during the later part of T2. If so, examination of exploration in only the first 30 s or 60 s would reveal differences in exploration of familiar and novel object which might have been obscured when data from the entire 3 minute duration was analysed. Figure 3.4 shows the time spent exploring familiar and novel objects during the first 30s (above) and 60s (below) of T2. 


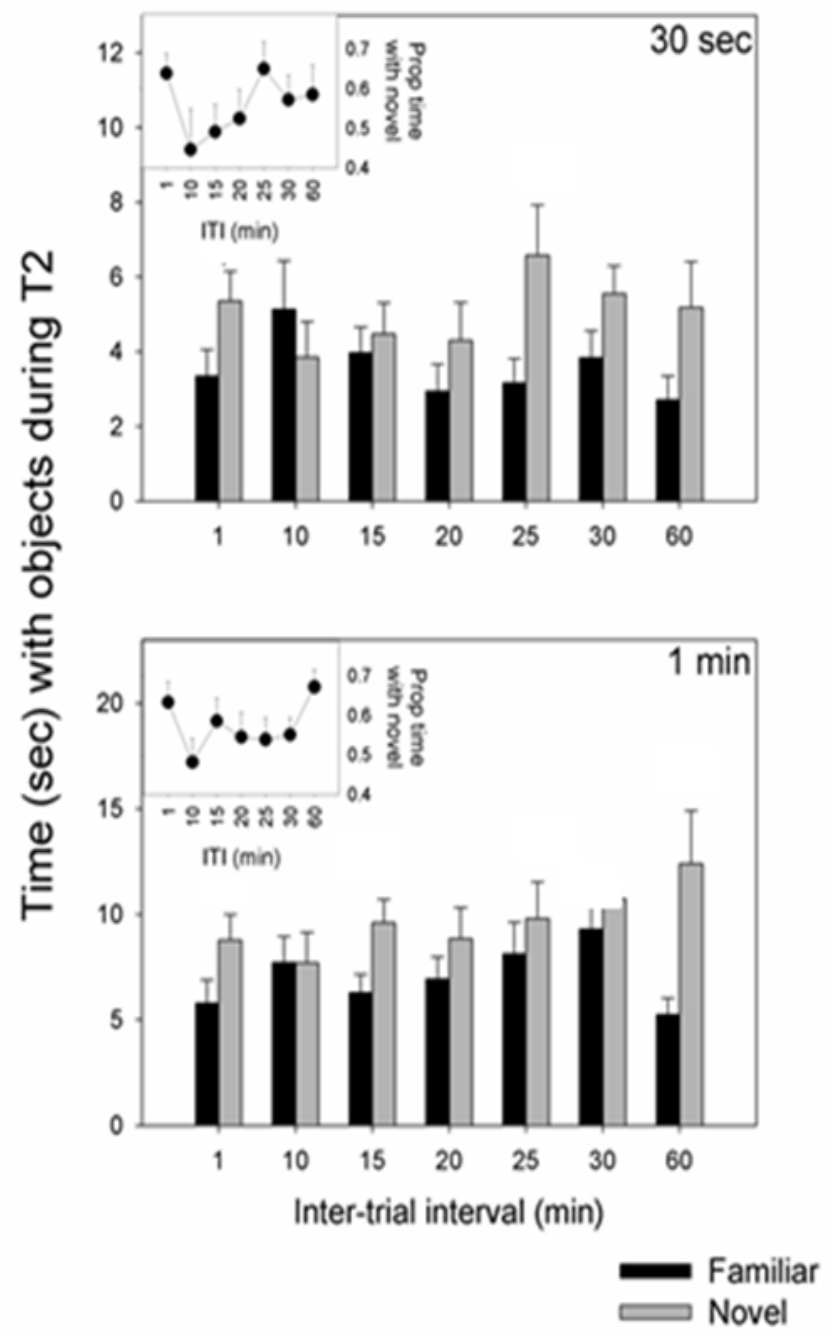

Figure 3.4 Time (in seconds) spent exploring familiar and novel objects during T2 when exploration was restricted to either $30 \mathrm{~s}$ (top) or $60 \mathrm{~s}$ (bottom). Bars represent mean time (+SEM). Inserts show the mean (+SEM) proportion of time with the novel object, $* \mathrm{p}<0.05$

Figure 3.5 shows the proportion of time spent with the duplicate (T1) and novel (T2) objects as a function of ITI when data from either the first 30 $\mathrm{s}$ (top) or $60 \mathrm{~s}$ (bottom) of $\mathrm{T} 2$. Thus, these data take into consideration any initial position bias during T1 that might impact the data. For the $30 \mathrm{~s}$ $(\mathrm{F}(1,81)=74.19, \mathrm{p}<0.01)$ and $60 \mathrm{~s}(\mathrm{~F}(1,81)=11.856, \mathrm{p}<0.01)$ data, ANOVA (trial X ITI) revealed a significant effect of trial and a significant interaction between ITI and test was observed for the $30 \mathrm{~s}$ data $(F(6,81)=64.4, \mathrm{p}<0.01)$. 

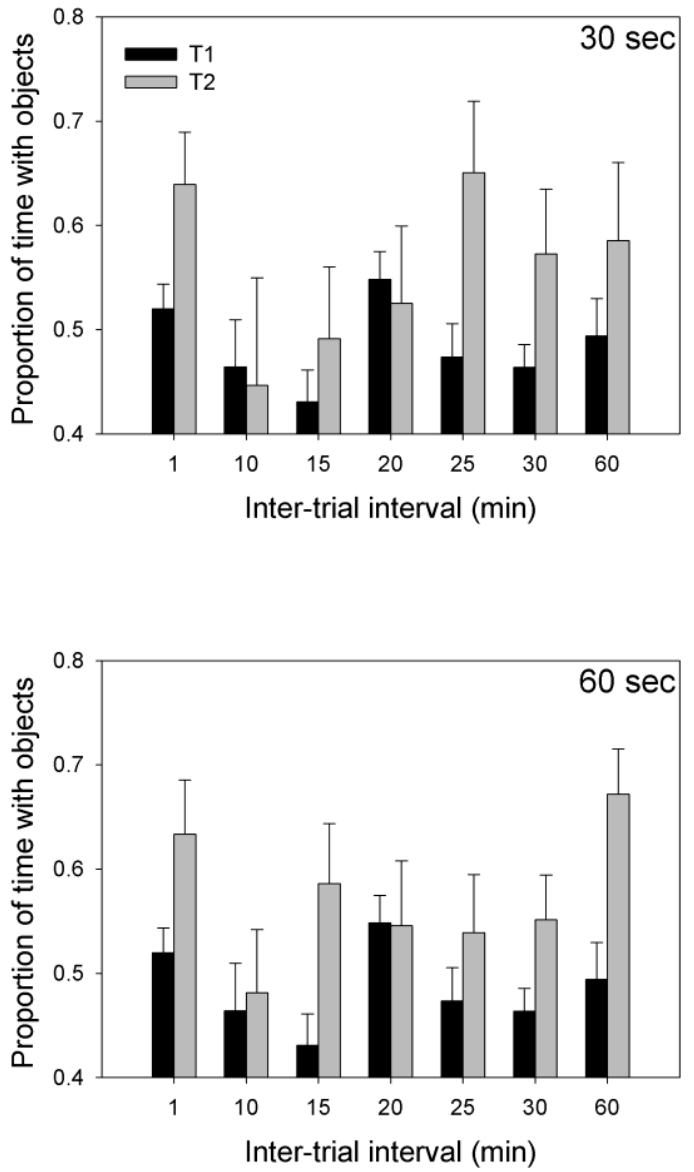

Figure 3.5 Mean proportion of time (+SEM) spent with the duplicate (in T1) and novel (in T2) object as a function of intertrial interval when exploration was restricted to the first $30 \mathrm{~s}$ (top) or $60 \mathrm{~s}$ (bottom) of T2.

Figure 3.6 (top) shows that the number of approaches to the two identical objects in T1 was comparable across all of the ITIs. There was no indication of side preference. From figure 3.6, (bottom), it appears as though there is a marked preference for the novel object, as indicated by a higher number of approaches, following the 1 minute and 10 minutes ITIs. Thereafter, the number of approaches to the two objects was comparable. These data are consistent with a forgetting curve following ITIs of 1-20 min. ANOVA revealed an effect of test $(F(1,203)=10.294$, $p<0.05)$, but no effect of ITI $(F(6,203)=1.45, N S)$, and no interaction $(F(6,203)=2.02, N . S)$. 

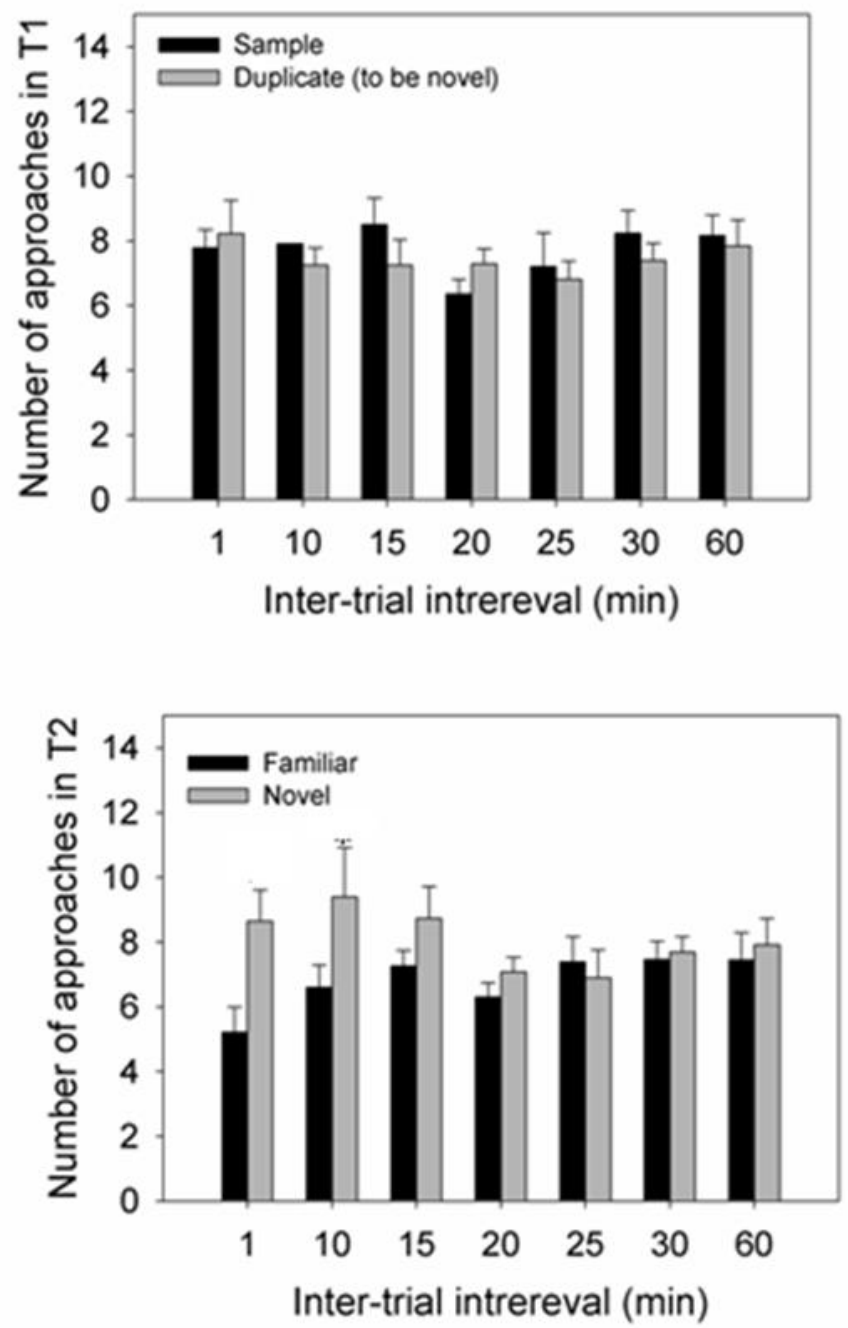

Figure 3.6 Mean number of approaches (+SEM) to objects during T1 (top) and $\mathrm{T} 2$ (bottom),

Figure 3.7 compares the data from $\mathrm{T} 1$ and $\mathrm{T} 2$ and shows the proportion of approaches to the duplicate (T1) and novel (T2) objects as a function of ITI. Thus, these data take into consideration any initial position bias during $\mathrm{T} 1$ that might have an impact on the data. There was a rapid decline in approaches as a function of ITI.ANOVA revealed an effect of ITI 
$(\mathrm{F}(6,206)=3.67, \mathrm{p}<0.05)$, trial $(\mathrm{F}(1,206)=10.23, \mathrm{p}<0.05)$ and an interaction $(\mathrm{F}(6,206)=1.94, \mathrm{p}=0.70)$.

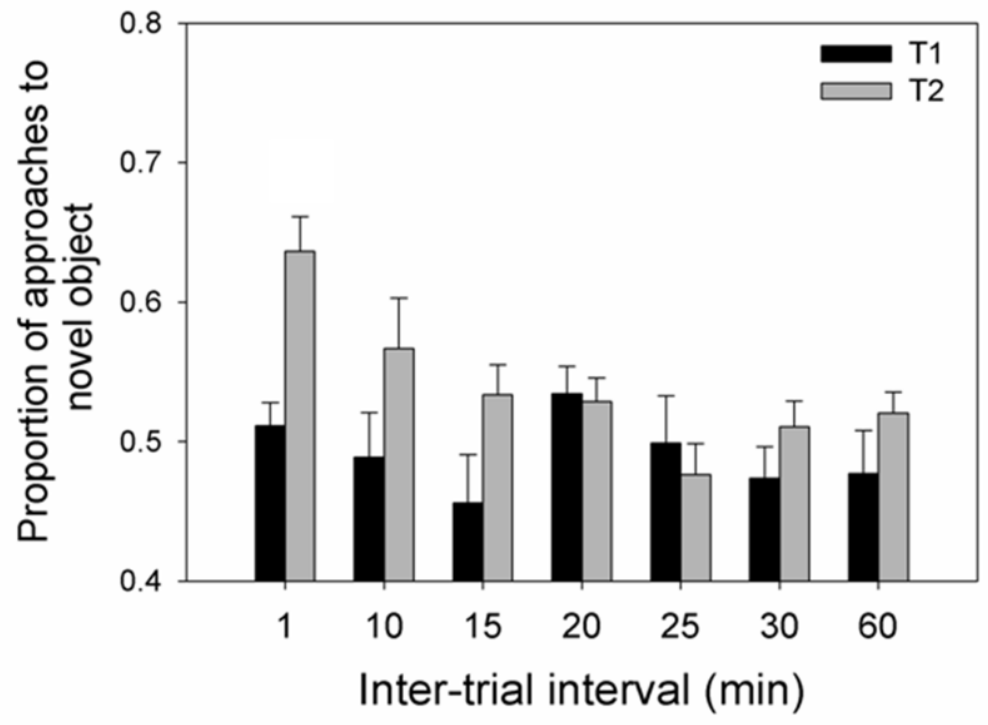

Figure 3.7 Mean proportion of approaches (+SEM) to the duplicate (in T1) and novel (in T2) object as a function of ITI

\section{$\underline{\text { Discussion }}$}

The purpose of this study was to establish the parameters for the NOR task. A range of ITIs was used in order to establish a forgetting curve in drug-naïve rats which could then be compared to that produced by MDMA treatment. Furthermore, a comparison between the use of exploration time versus number of approaches to the objects for analyses was carried out.

A forgetting curve was not produced when exploration time of the novel object was plotted as a function of ITI. There was, however, a decay of preference for the novel object as measured by exploration time at ITIs longer than $1 \mathrm{~min}$, which then manifested again after an ITI of $60 \mathrm{~min}$. Because of this sporadic finding, it seems unlikely that the time spent with the novel vs familiar object in $\mathrm{T} 2$ was an indication of recognition memory. 
Regardless of whether data were collected from the first 30 s or 60 s of $\mathrm{T} 2$, or the entire 3 minutes, there was more exploration of the novel object following an inter-trial interval of $1 \mathrm{~min}$ and $60 \mathrm{~min}$. The difference in exploration time for the two objects was, at best, 5-6 s of the $3 \mathrm{~min}$ exploration period (see Figure 3.1). Assuming that this was a meaningful difference, these findings would suggest that NOR memory decays by 20 min and then re-emerges following a 60 min delay.

It is possible that handling at the start of the T2 sessions interfered with exploration, especially when the ITI was short. It is difficult, however, for this potential confound to explain the increased exploration of the novel object following the $1 \mathrm{~min}$ ITI. Rather, the data are not consistent with the idea that exploration time under these experimental conditions is a good reflection of NOR.

Studies in infants have also shown varying novel object exploration as a function of delay between the familiarization and test phases when exploration time was measured. In the human studies, initial preferential exploration of the novel object was produced following short delays (1 min), followed by null preferences in exploration following an intermediate delay (1 day to 1 month) and preference for a familiar object, termed remote memory, with yet further increases in delay (1-3 months; Bahrick and Pickens, 1995). The present data show a different profile in that a novelty preference was suggested following both short or long retention intervals but null preferences were produced when intermediate ITIs were imposed. Regardless, both the infant studies and the rat study reported here suggest that time spent exploring an object is not always an appropriate measure of memory.

Other studies (see, for example, Ludwig et al, 2008; Morley et al, 2001; Piper and Meyer, 2004) have demonstrated a preference for the novel object following the 15 or 30 min ITIs when time spent exploring the objects was measured. It is not clear why NOR was not produced following these intervals but it might be related to habituation times or some other paradigmatic differences. Indeed, the magnitude of NOR is influenced by variables such as duration of the sample period (Ainge et al, 2006; Albasser et al., 2009; Ennaceur \& Delacour, 1988) and habituation time (Bevins and 
Besheer, 2006). Whatever the basis, the present results point to the importance of testing a range of intervals and having multiple dependent measures.

Ennaceur (2010) discussed the impact which factors like object affordance can have on the rodent's exploratory behaviour. Certain objects have properties which are more suited to allow rats to carry out every day activities (eg climbing) and therefore produce a natural bias (Chemero \& Heyser, 2012). In the current experiment, objects were chosen to be easily distinguishable from each other without bearing any features that could be construed as resembling anything with natural significance or reinforcing properties. Data from T1 suggest that none of the objects produced any obvious bias as exploration times are relatively consistent across intervals. It is, however, possible that this bias only manifested during T2, when choices between objects had to be made. This may account for the results obtained for the function of object recognition against ITI when exploration time was used.

Potentially, the use of number of approaches as a measure of exploration represents a 'purer' form of NOR and isolates only preferences based on memory. Another possibility is that different objects may require more exploration time in order to be encoded them. As mentioned before, these differences may be subtle and only become apparent when rats are presented with a choice between two objects. If these differences manifest mainly through duration of bouts of exploration, effects would be obscured by using number of approaches rather than total exploration time as a measure of exploration. In order to test across several intervals in the current experiment, 14 different pairs of objects which meet the above two criteria were required. In an attempt to achieve the first criterion of all objects being easily distinguishable, there was quite a large variation in the type of object used. Therefore, differences in object affordances or exploration strategies may have been somewhat unavoidable. Using number of approaches as a dependent measure may circumvent this issue.

Had we tested only the short and long intervals, we would have found results comparable to other studies that tested NOR after an ITI of $1 \mathrm{~h}$ or longer. Our data, based on analysis of effects following a larger number 
of short ITIs, suggest that exploration time might be an inappropriate measure. There are indeed difficulties in actually assigning accurate exploration time either when automated or manual means of assessment are used (Ennaceur, 2010). It can be difficult to ascertain whether the rat is actually exploring from a distance of 1-2 $\mathrm{cm}$ and often this becomes a subjective rather than objective measure that can lead to variable and inaccurate results. Indeed, we found that when several experimenters who were blind to the conditions scored random tapes, inter-rater reliability was low. The less biased measure of frequency of approaches has been suggested to be particularly sensitive to differences in NOR across rat strains and gender (Ennaceur, 2010; Ennaceur et al., 2005) and so we applied this to the NOR data and found it to be a much more reliable measure.

Unlike the preference data obtained when time spent exploring the items was used, a profile consistent with a forgetting curve was produced when the number of approaches was analysed. Unlike the apparent but unlikely remembering that emerged following the $60 \mathrm{~min}$ ITI when exploration time was measured, number of approaches produced chance levels of exploration following ITIs greater than or equal to 15-20 min. Object recognition memory in rats, when measured in paradigms such as DNMS or DMTS, decays rapidly after relatively short durations, in the order of tens of seconds (For example see Aura et al, 1997; Money et al, 1992; Rothblat \& Hayes, 1987). However, when the NOR paradigm is used, it has been suggested that memory remains intact over much longer intervals (between 1-24 hours; Lieben et al, 2004; 2005; Vannucchi et al, 1997; He et al, 2006; Clark et al, 2007; Ennaceur et al, 1988). In the current study, NOR was no longer apparent when the number of approaches was measured after 10 minutes. The discrepancies between these findings and others could potentially be due to strain differences (Ennaceur et al, 2005), or other paradigmatic variables.

In Sprague-Dawley rats, some studies have suggested that object recognition memory was retained for up to 3 hours (Bertaina-Anglade et al, 2006). The exploration time during T1 was only 20 s, which is substantially less than in the current study. However, there was no difference in NOR 
following an ITI of $10 \mathrm{~min}$ and $2 \mathrm{hrs}$. Deterioration of NOR over the first 2 hours would be expected on the basis of other studies (Able et al, 2006; McGregor et al, 2001). Therefore, the measure of NOR may not be a very reliable indicator in this study.

The current study, however, produced a forgetting function of NOR following short ITIs ranging from $1 \mathrm{~min}$ to 60 mins. Furthermore, we explored a novel method of analysis for this task, number of approaches, which more accurately reflects NOR under the present experimental conditions. This measure of exploratory behaviour may be useful for measuring NOR when the task is established over a range of ITIs. 


\title{
Chapter 4: The effects of MDMA self-administration on NOR
}

\author{
(These findings have been published in Schenk S., Harper D. \& Do (2010) Novel object \\ recognition memory: measurement issues and effects of MDMA self-administration \\ following short inter-trial intervals. Journal of Psychopharmacology, 25: 1043-1052. Do's \\ contribution was data collection, data analysis and some of the manuscript writing)
}

\section{Overview}

After establishing a forgetting function for NOR in control rats, NOR was tested 7 or 70 days following MDMA self-administration (total dose of $165.0 \mathrm{mg} / \mathrm{kg}$ ). Cognitive deficits have been demonstrated in laboratory animals between 1 and 2 weeks following exposure (Sprague et al, 2003; Rodsiri et al, 2010). Therefore, 7 days and 70 days were chosen in order to determine whether MDMA self-administration produced deficits in NOR that recovered over time. Controls for the 70 day group were exposed to self-administration chambers for daily 2 hour session (6 days a week, for 4 weeks) and testing was conducted 70 days following the last session in the self-administration chamber.

$\underline{\text { Results }}$

MDMA self-administration

Of the original cohort of rats $(n=30), 60 \%$ met the criterion for MDMA self-administration and went on to be tested in the NOR task. An average of 28.4 days ( \pm 1.7 days; range $21-41$ days) and 23.9 days ( \pm 1.8 days; range 13-30 days) was required to meet the criterion $165.0 \mathrm{mg} / \mathrm{kg}$ for the groups tested 7 days $(n=11)$ and 70 days $(n=8)$, respectively. Figure 4.1 
shows the frequency distribution of the number of days to meet the initial criterion of self-administration of $90.0 \mathrm{mg} / \mathrm{kg} / \mathrm{infusion}$. The distribution is positively skewed and between 16-20 days was the modal. Inactive lever presses remained low during testing.

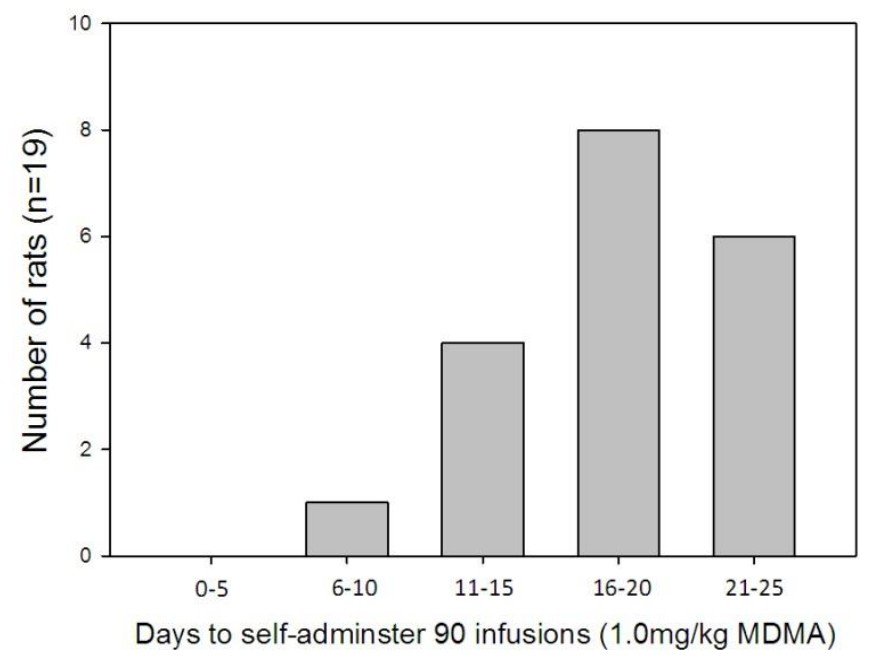

Figure 4.1 Frequency distribution of number of days to self-administer 90 infusions of $1.0 \mathrm{mg} / \mathrm{kg}$ MDMA.

The average daily intake during the first 3 days of testing for rats that were tested 7 days following self-administration was $1.7 \pm 0.6 \mathrm{mg} / \mathrm{kg}$, and this increased to $9.1 \pm 1.0 \mathrm{mg} / \mathrm{kg}\left(\mathrm{t}(20)=6.50,{ }^{*} \mathrm{p}<0.05\right)$ during the last 3 days of self-administration of $1.0 \mathrm{mg} / \mathrm{kg} /$ infusion (Figure 4.2 ). When the dose was decreased to $0.5 \mathrm{mg} / \mathrm{kg} /$ infusion, the average daily intake over the first and last 3 days of self-administration was $6.8 \pm 0.3 \mathrm{mg} / \mathrm{kg}$ and $10.5 \pm$ $0.8 \mathrm{mg} / \mathrm{kg}\left(\mathrm{t}(20)=2.97,{ }^{*} \mathrm{p}<0.05\right)$ respectively. 

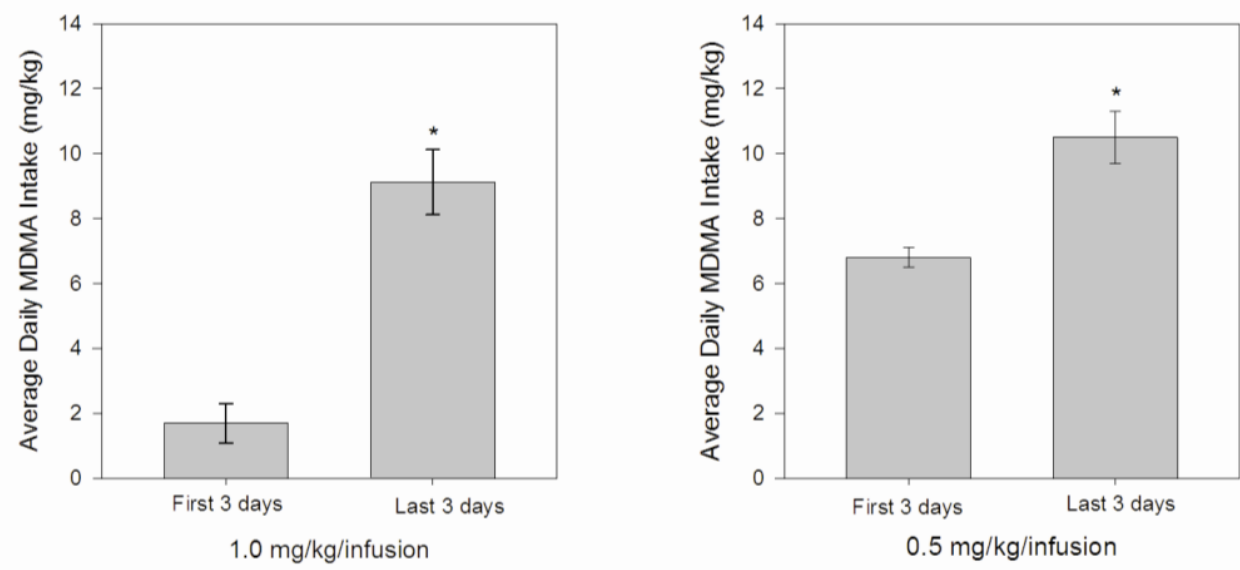

Figure 4.2 Average daily intake $( \pm$ SEM) during the first and last 3 days of self-administration of $1.0 \mathrm{mg} / \mathrm{kg} / \mathrm{infusion}$ and $0.5 \mathrm{mg} / \mathrm{kg} / \mathrm{infusion}$ in rats tested 7 days following the last self-administration session $* \mathrm{p}<0.05$ compared to first 3 days

For rats tested 70 days following the last self-administration session, the average daily intake during the first 3 days of self-administration of 1.0 $\mathrm{mg} / \mathrm{kg} / \mathrm{infusion}$ was $3.8 \pm 1.3 \mathrm{mg} / \mathrm{kg}$, and this increased to $12.4 \pm 2.0 \mathrm{mg} / \mathrm{kg}$ $(\mathrm{t}(14)=3.55, \mathrm{p}<0.05)$ during the last 3 days of self-administration. When the $0.5 \mathrm{mg} / \mathrm{kg} /$ infusion dose was available, the average daily intake during the first 3 and last 3 days was $10.9 \pm 2.4 \mathrm{mg} / \mathrm{kg}$ and to $12.4 \pm 0.7 \mathrm{mg} / \mathrm{kg}$ (t $(14)=0.78, \mathrm{NS})$ respectively. In total, rats that were tested 7 days or 70 days following exposure self-administered $169.9 \pm 2.0 \mathrm{mg} / \mathrm{kg}$ and $172.3 \pm 3.1$ $\mathrm{mg} / \mathrm{kg}$ MDMA, respectively. 

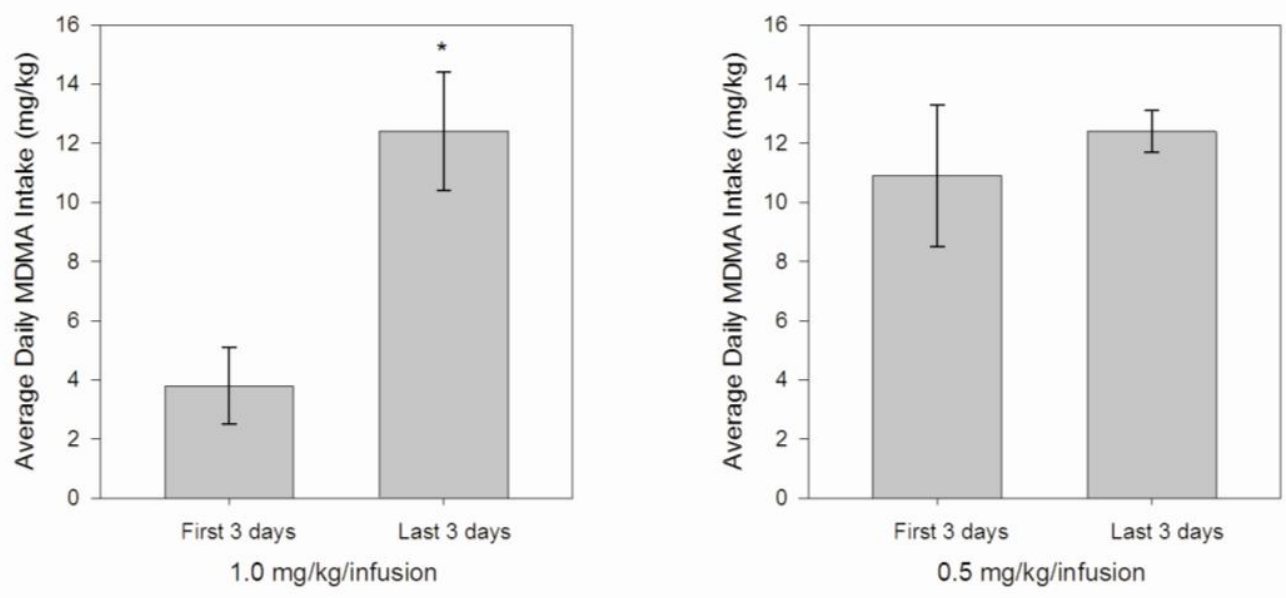

Figure 4.3 Average daily intake $( \pm$ SEM) during the first and last 3 days of self-administration of $1.0 \mathrm{mg} / \mathrm{kg} / \mathrm{infusion}$ and $0.5 \mathrm{mg} / \mathrm{kg} / \mathrm{infusion}$ in rats tested 70 days following the last self-administration session, $* \mathrm{P}<0.05$ compared to first 3 days

NOR testing

NOR testing 7 days following self-administration

Figure 4.4 shows the exploration times of objects as a function ITI during T1 and T2 when testing was conducted 7 days following selfadministration. The total amount of exploration time during T1 and T2 was generally comparable to that observed in the control group, ranging between 30s and 45s. MDMA self-administration did not have any effect on general exploratory behaviour. During T1, there was no obvious bias except for during the 25 minute ITI in which subjects generally spent more time with the duplicate object than the sample object. 

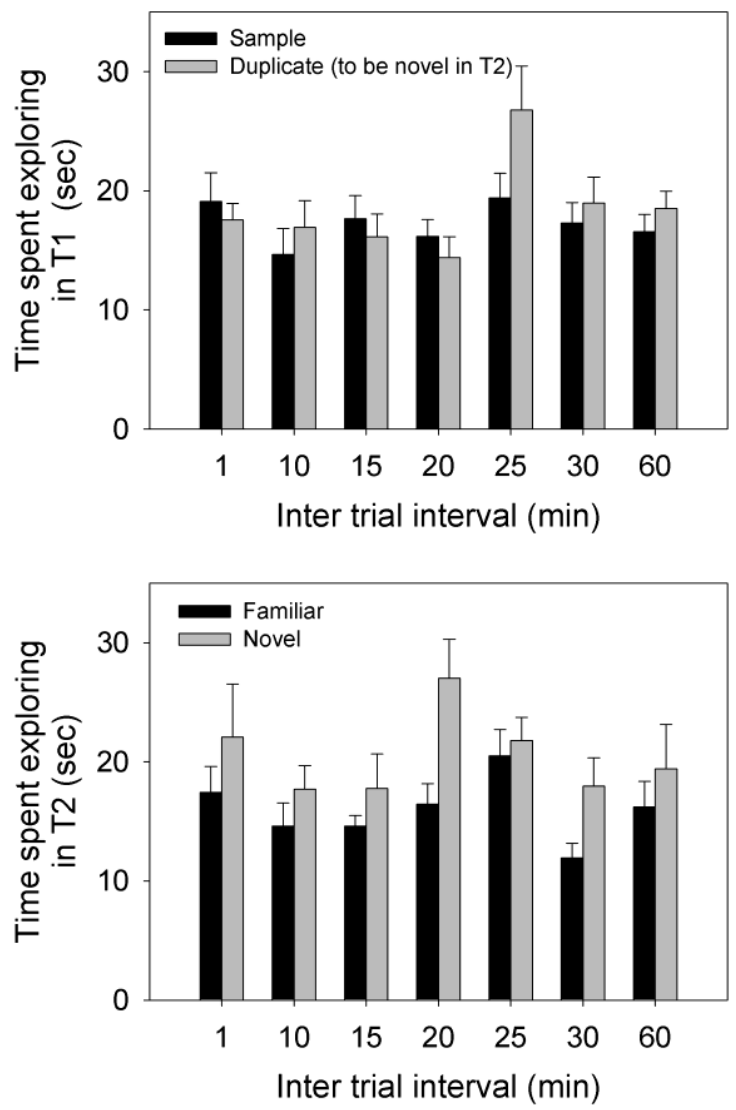

Figure 4.4 Time (in seconds) spent exploring objects during T1 (top) and T2 (bottom) as a function of inter trial interval for subjects that selfadministered MDMA. Data were collected 7 days following the last selfadministration session. Bars represent mean time (top) or mean proportion of time (bottom) (+SEM).

Figure 4.5 shows the number of approaches to sample and duplicate objects during T1. The number of approaches to each of the objects during each ITI was similar suggesting a lack of any obvious bias. Furthermore, the total number of approaches fell within the range of that observed for the control group. There was also no difference in the number of approaches to familiar or novel object following all of the ITIs during T2 (Figure 4.5). 

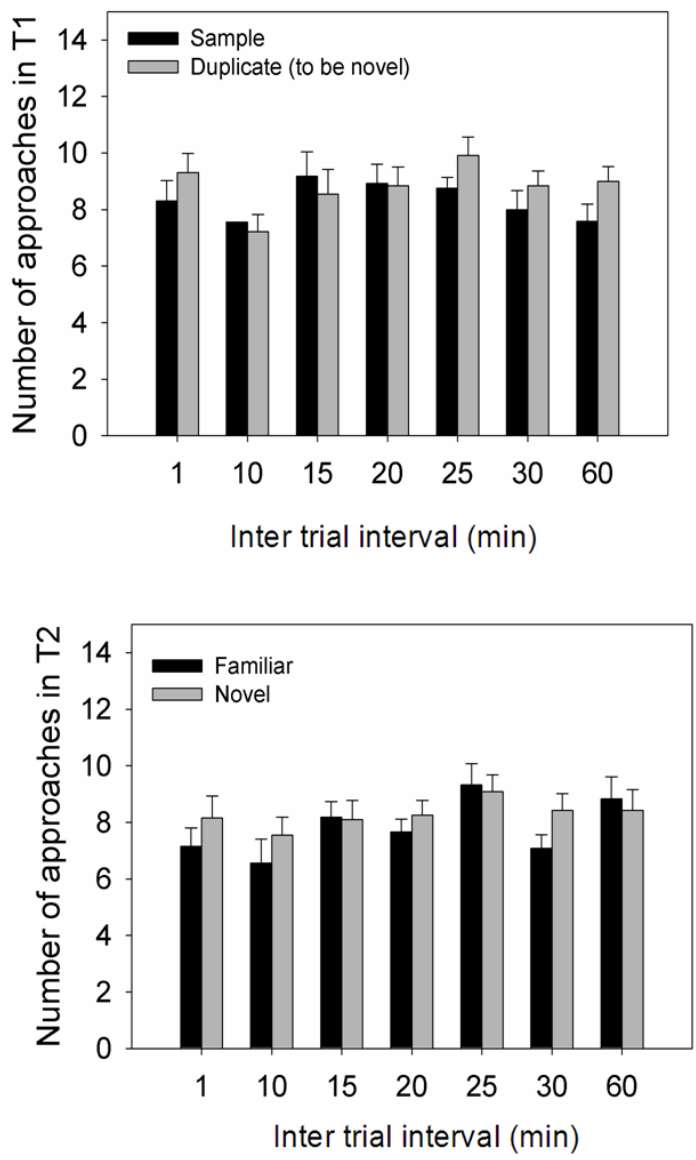

Figure 4.5. Mean number of approaches (+SEM) to objects during T1 (top) and T2 (bottom) for subjects that self-administered MDMA. Data were collected 7 days following the last self-administration session.

When proportion of approaches to novel objects during T2 was compared in MDMA-self-administering and control rats, ANOVA revealed an effect of ITI $(\mathrm{F}(96,207)=3.22, \mathrm{p}<0.05)$, an interaction $(\mathrm{F}(6,207)=2.18$, $\mathrm{p}<0.05)$ but no effect of treatment $(\mathrm{F}((1,207)=0.87$, NS $)$. Following at ITI of 1 minute, the proportion of approaches to the novel object was higher in controls than MDMA self-administering subjects $(\mathrm{t}(24)=2.398, \mathrm{p}<0.05)$. There were no group differences at any other ITIs $(p>0.05)$. 


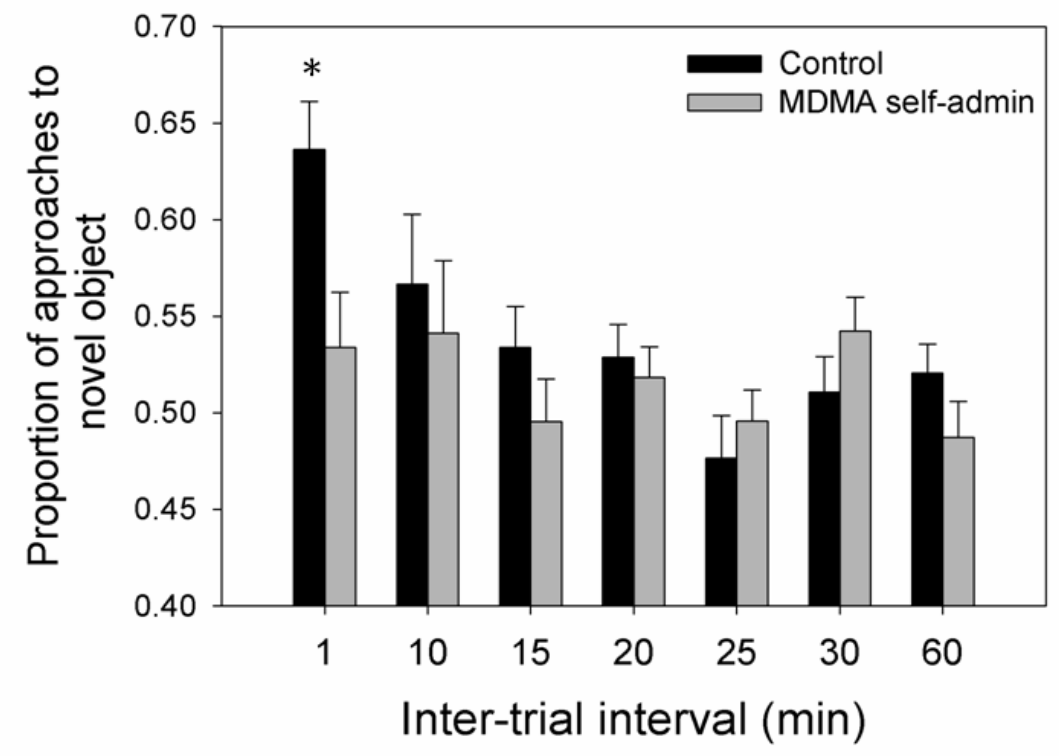

Figure 4.6. Mean proportion of approaches (+SEM) to the novel object as a function of inter-trial interval for control subjects and subjects that selfadministered MDMA. Data were collected 7 days following the last selfadministration session.

NOR testing 70 days following self-administration

Data from control subjects tested 7 days and 70 days following last session were comparable (Figure 4.5 \& Figure 4.6). During T1, the number of approaches to sample and duplicate objects was similar across all ITIs and the total number of approaches was not markedly different. This would suggest that there is no observable bias and also importantly, that the long period of 10 weeks had little effect on the exploratory behaviour of control subjects. Figure 4.7 shows the number of approaches to both familiar and novel object during T2 across all ITIs in control subjects tested at 70 days. The function decayed in a manner similar to that seen in control subjects tested at 7 days.

In subjects that self-administered MDMA, there appears to be a recovery of NOR. The exploratory behaviour of the subjects was not affected by either MDMA self-administration or the 10 weeks between testing (Figure 4.8). The total number of approaches to objects during T1 
and $\mathrm{T} 2$ is comparable to the data attained from control data. However, during $\mathrm{T} 2$, there are more approaches to the novel object than the familiar object following an ITI of both 1 minute $(\mathrm{t}(4)=2.93, \mathrm{p}<0.05)$ and 10 minutes $(\mathrm{t}(4)=5.72, \mathrm{p}<0.05)$.
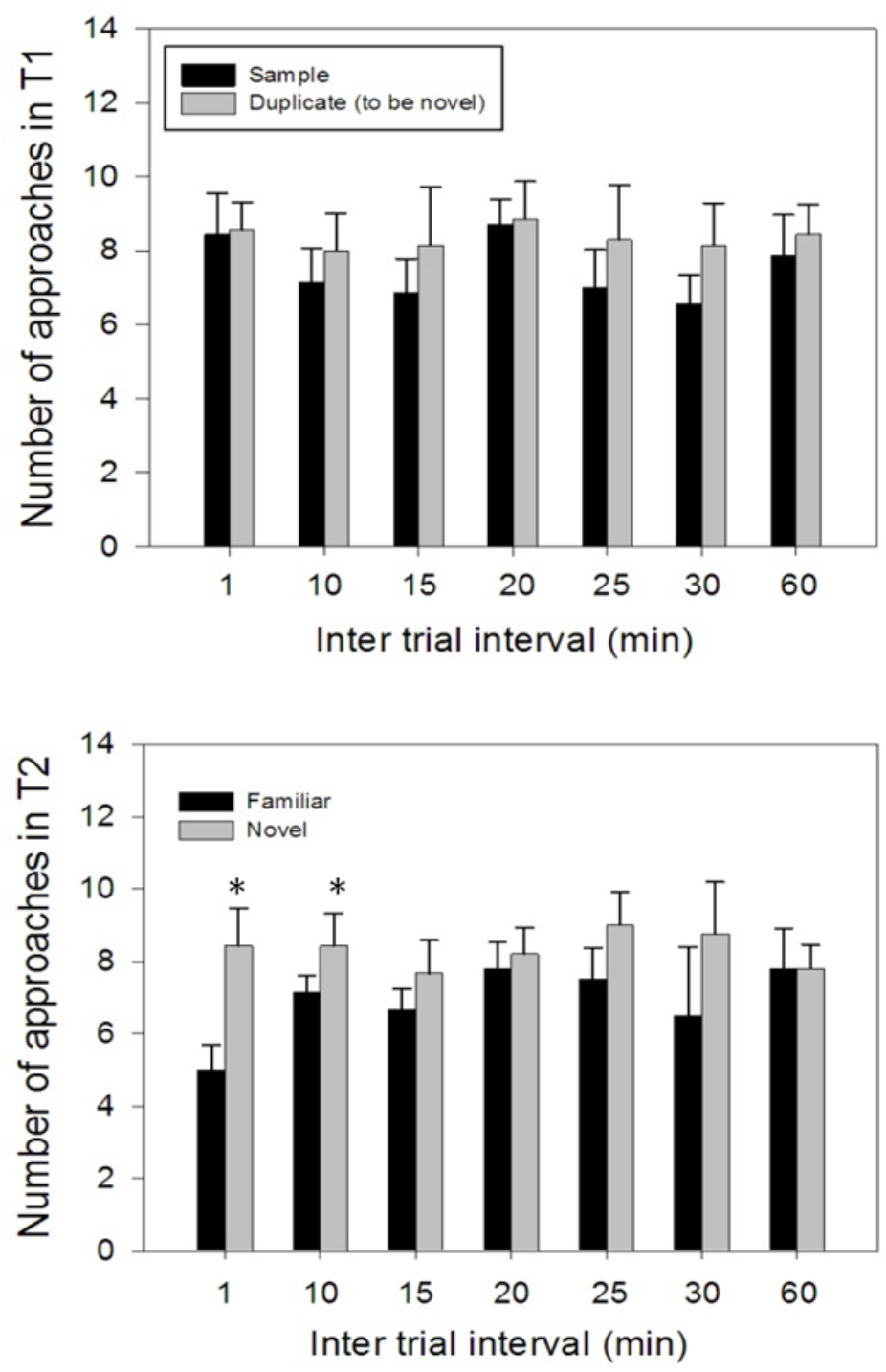

Figure 4.7 Mean number of approaches (+SEM) to objects during T1 (top) and T2 (bottom) for control subjects. Data were collected 70 days following the last session in self-administration chambers. ${ }^{*} \mathrm{p}<0.05$

When comparing the proportion of approaches to the novel object during T2, the data for the subjects which self-administered MDMA is comparable to the age-matched controls. ANOVA revealed an effect of ITI 
$(\mathrm{F}(6,72)=4.25, \mathrm{p}<0.05)$, but no effect of treatment $(\mathrm{F} 1,72)=1.02$, NS $)$ or interaction $(\mathrm{F}(6,72)=2.13, \mathrm{NS})$.

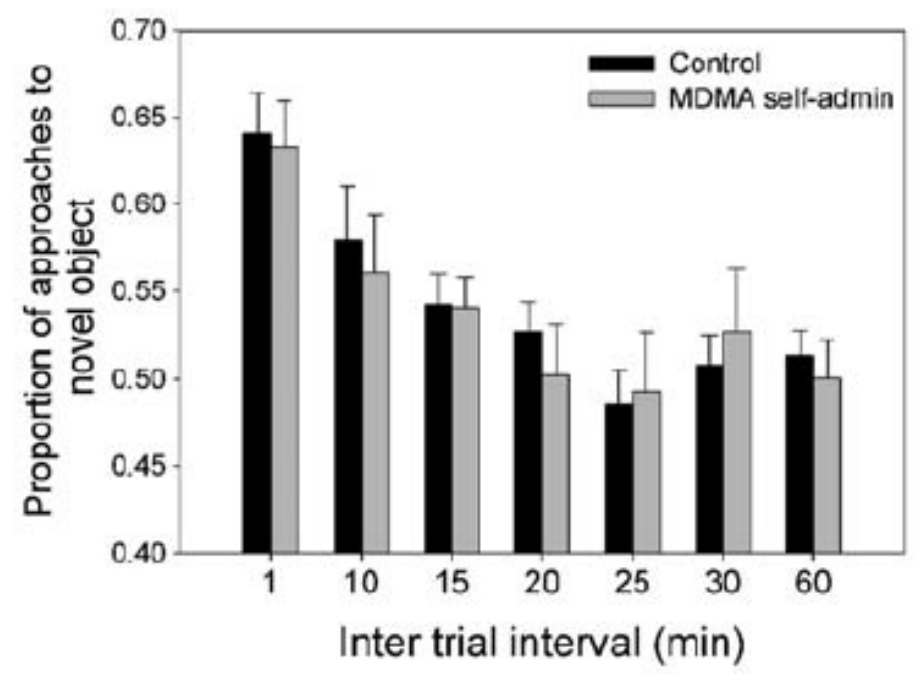

Figure 4.8 Mean proportion of approaches (+SEM) to the novel object as a function of inter-trial interval for control subjects and subjects that selfadministered MDMA. Data were collected 70 days following the last selfadministration session.

\section{$\underline{\text { Discussion }}$}

There was a deficit in NOR performance 7 days, but not 70 days, following MDMA self-administration. It might be important that control subjects did not undergo the same surgical procedure as MDMA selfadministering subjects. It is possible that the manipulations, anaesthetics or other factors related to the surgery may have had an influence on the performance in the NOR task. This, however, is unlikely since the 10 week MDMA self-administration rats underwent all of the surgical procedures but failed to show the deficit.

This study demonstrates a deficit in NOR induced by MDMA selfadministration which recovered over time. Studies have previously measured performance in the NOR task following administration of bingelike regiments of MDMA (Able et al, 2006; Camarasa et al, 2008; Morley et al, 2001; Piper \& Meyer, 2004) and MDMA produced a dose-dependent 
impairment in NOR. However, methodological issues with the NOR paradigm in these studies render the data difficult to interpret.

One significant issue relates to the failure to observe ITI-dependent effects. In the current study, we attempted to resolve this issue by establishing a forgetting function in control subjects, described in Chapter 3. MDMA self-administration impaired performance, but we cannot necessarily attribute the deficit to disruptions of memory processes, since a forgetting function for the MDMA self-administering subjects was not produced. A leftward shift in the forgetting curve would have been a reliable indication that NOR was disrupted. Instead, exploration of the novel object during T2 was at chance level at all of the ITIs tested, 7 days following the last self-administration session.

The possibility that the deficit observed was due to impairment of other cognitive processes including attention and impulsivity cannot be ignored. A recent study (Dalley et al, 2007) showed that MDMA selfadministration produced deficits in the five-choice-serial-reaction-time task that were consistent with abnormalities in attentional and motivational functions as well as increased impulsivity in rats. Because exploration of objects during T1 and T2 remained intact 7 days following MDMA selfadministration, it is doubtful that motivational or attentional processes can explain the data. On the other hand, cognitive processes which support encoding of objects during $\mathrm{T} 1$ or even the natural bias towards novel objects may have been disrupted. Also, the forgetting curve for the MDMA selfadministering subjects tested 7 days later may have been difficult to observe due to a 'floor effect' at ITIs equal to or longer than 1 minute. Preference for the novel object in controls at ITIs longer than 1 minute fell below 55\% and therefore any deficit in NOR due to MDMA self-administration at these intervals would be difficult to detect. Regardless, these findings demonstrate that MDMA self-administration produces cognitive deficits which recover over time and support findings that cognition is impaired in MDMA users (De Sola et al, 2008; De Sola 1lopis et al, 2008; Reneman et al, 2001; Schilt et al, 2010; Waering et al, 2004). Evidence suggests that MDMA-produced deficits in learning and memory may be due to changes in 5HT neurotransmission in humans (Reneman et al, 2000) and this is supported by 
a well-established role of the 5HT system in learning and memory (Ogren et al, 2008; Meneses et al, 2011; Codony et al, 2011; Gonzalez-Burgos \& Feria-Velasco, 2008). NOR deficits following MDMA self-administration, therefore, may reflect a compromised 5HT system. In order to investigate this possibility, tissue 5HT levels were measured 2 and 10 weeks following the last MDMA self-administration session. 


\section{Chapter 5: Dose- and time-dependent effects of MDMA self- administration on tissue levels of 5HT}

(These findings have been published in Do, J. \& Schenk S. (2011) Self-administered MDMA produces dose- and time- dependent serotonin deficits in the rat brain. Addiction Biology, doi: 10.1111/j.1369-1600.2011.00370.x. [Epub ahead of print]

\section{Overview}

A number of studies have shown that tissue levels of 5HT are reduced 2 weeks following experimenter-administered MDMA (Stone et al, 1987; Scanzello et al, 1993; Sabol et al, 1996). Effects of self-administered MDMA, however, have not been well established. Therefore, the effect of MDMA self-administration on 5HT tissue levels in the frontal cortex, striatum and hippocampus 2 weeks following drug exposure was determined in the present study. Because at least partial recovery occurred between 2 and 16 weeks following exposure (Stone et al, 1987; Scanzello et al, 1993; Sabol et al, 1996), and because results of Chapter 4 suggested recovery of NOR, tissue levels of 5HT were also measured following an abstinence period of 10 weeks.

For these experiments, 2 different doses of MDMA were selfadministered; $165.0 \mathrm{mg} / \mathrm{kg}$ and $315.0 \mathrm{mg} / \mathrm{kg}$. A dose of $165.0 \mathrm{mg} / \mathrm{kg}$ was chosen as it had previously been reported to reduce tissue levels of 5HT in the frontal cortex, striatum and hippocampus 5 days following selfadministration (Schenk et al, 2010). The effect of a higher dose of 315.0 $\mathrm{mg} / \mathrm{kg}$ was tested to determine whether greater or more persistent effects would be produced. 
$\underline{\text { Results }}$

\section{Self-administration}

Of the original cohort of rats $(n=70), 50 \%$ completed either the low or high intake criterion. The average number of days required to selfadminister 90 infusions of $1.0 \mathrm{mg} / \mathrm{kg}$ MDMA was $16.9 \pm 0.8$. Figure 5.1 shows the frequency distribution of the number of days required to meet this criterion. The distribution is positively skewed and extended testing (>10 days) was generally required to meet the criteria (Figure 5.1). Only two subjects self-administered the 90 infusions in less than 10 days.

The average number of days required to self-administer 90 infusions of $1.0 \mathrm{mg} / \mathrm{kg}$ MDMA in the high dose group was $19.6 \pm 1.3$ days and was significantly longer than the low dose group that required $15.1 \pm 0.9$ days $(t$ $(32)=2.97, \mathrm{p}<0.05)$. Once this initial criterion was met, the high dose group required an average of $17.4 \pm 0.9$ days to produce the subsequent 450 lever presses on $0.5 \mathrm{mg} / \mathrm{kg}$ MDMA while the low dose group required an average of $7.2 \pm 0.7$ days to self-administer to produce the 150 lever presses on 0.5 $\mathrm{mg} / \mathrm{kg} /$ infusion.

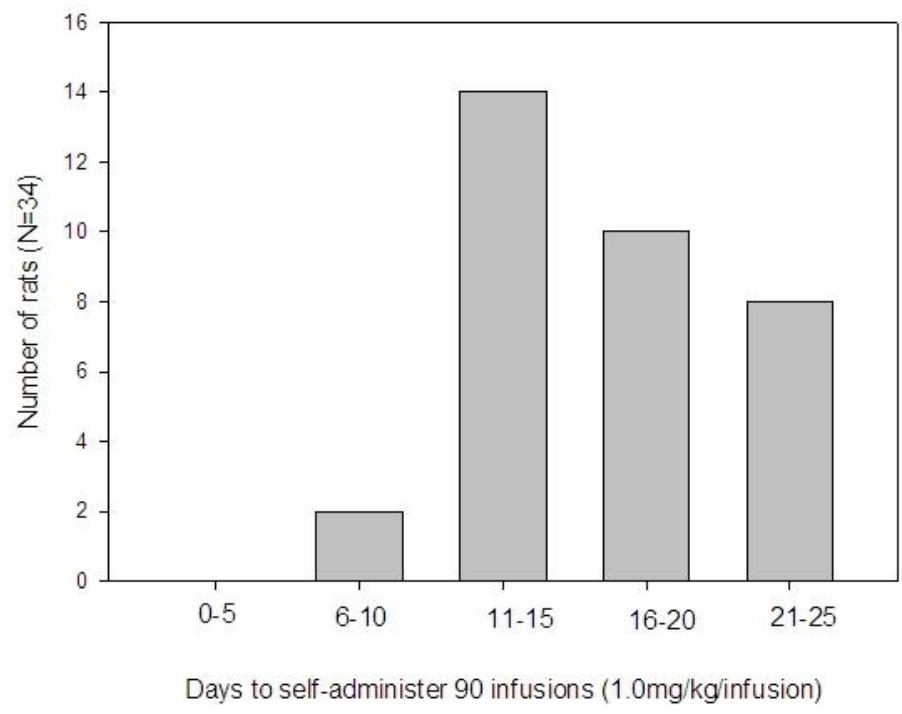

Figure 5.1 Frequency distribution of number of days required to selfadminister 90 infusions of $1.0 \mathrm{mg} / \mathrm{kg}$ MDMA 
Figure 5.2 shows the amount of self-administered MDMA (1.0 (left) or 0.5 (right) $\mathrm{mg} / \mathrm{kg} / \mathrm{infusion}$ ) as a function of test day. Small amounts of MDMA were self-administered during the first 3 days of testing (1.0 $\mathrm{mg} / \mathrm{kg} /$ infusion (average daily intake of $3.4 \pm 0.5 \mathrm{mg} / \mathrm{kg}$; Figure 5.2 ). With extended testing, intake increased and during the last 3 days of selfadministration of this dose $11.8 \pm 0.7 \mathrm{mg} / \mathrm{kg}(\mathrm{t},(67)=11.16, \mathrm{p}<0.05)$ was self-administered. When the dose was decreased to $0.5 \mathrm{mg} / \mathrm{kg} / \mathrm{infusion}$, responding increased in a compensatory manner to maintain intake (average daily intake of $11.3 \pm 0.8 \mathrm{mg} / \mathrm{kg}$ and $14.5 \pm 0.9 \mathrm{mg} / \mathrm{kg}$ during the first and last 3 days of self-administration, $(\mathrm{t},(67)=21.41, \mathrm{p}<0.05)$ respectively.
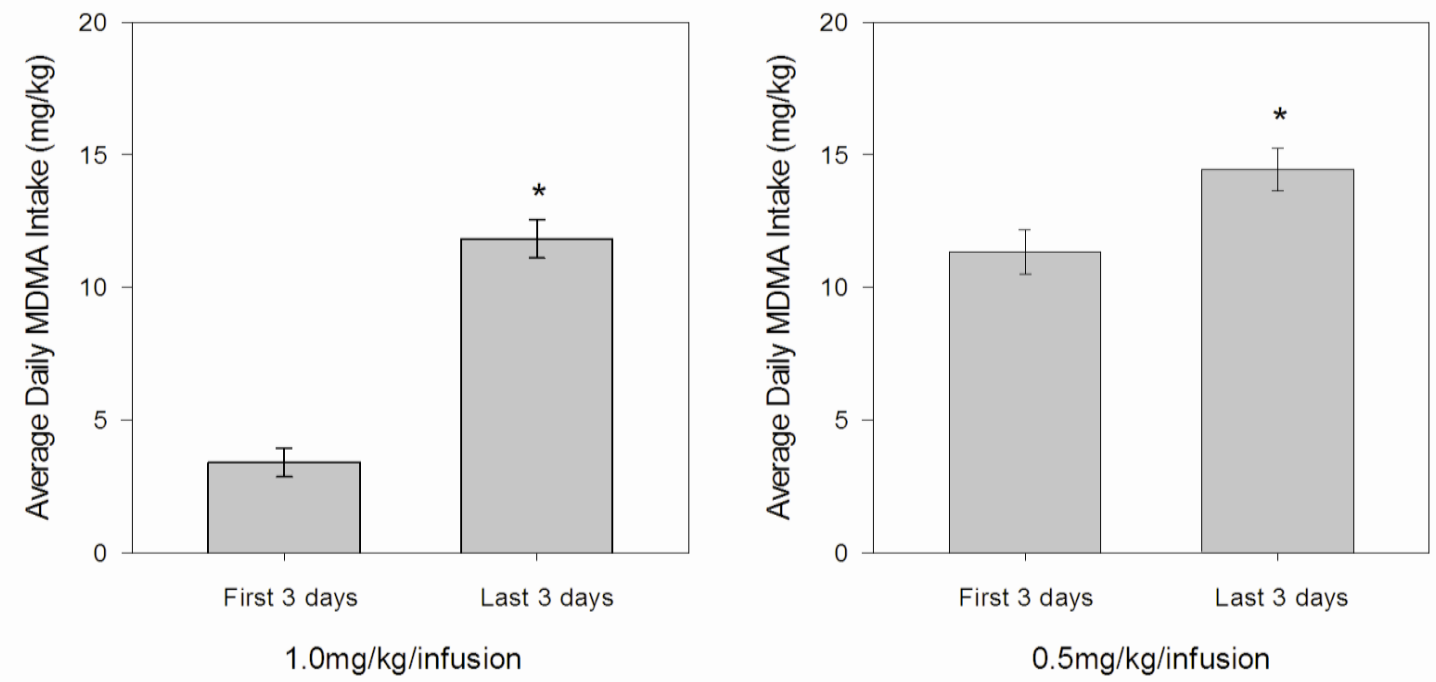

Figure 5.2 Average daily intake $( \pm$ SEM) during the first and last 3 days of self-administration of $1.0 \mathrm{mg} / \mathrm{kg} /$ infusion and $0.5 \mathrm{mg} / \mathrm{kg} /$ infusion doses, $* \mathrm{p}<0.05$

\section{Neurochemistry}

Control levels of 5HT ( 2 weeks) were $0.58 \pm 0.02 \mathrm{ng} / \mathrm{mg}, 0.54 \pm 0.04$ $\mathrm{ng} / \mathrm{mg}$ and $0.56 \pm 0.04 \mathrm{ng} / \mathrm{mg}$ in the frontal cortex, striatum and hippocampus, respectively. When assays were conducted 10 weeks after behavioural 
testing, tissue levels of $5 \mathrm{HT}$ in controls were $0.54 \pm 0.03 \mathrm{ng} / \mathrm{mg}, 0.52 \pm 0.04$ $\mathrm{ng} / \mathrm{mg}$ and $0.45 \pm 0.04 \mathrm{ng} / \mathrm{mg}$ in the frontal cortex, striatum and hippocampus. Tissue levels of 5HT in the hippocampus of the 10 week group were significantly lower than the 2 week group $(\mathrm{t}(32)=2.45, \mathrm{p}<0.05)$. In order to directly compare effects of MDMA self-administration across both time periods and across all sites, values were converted to percentage of control.

Two weeks following low dose $(165.0 \mathrm{mg} / \mathrm{kg})$ of MDMA selfadministration, 5HT levels were approximately $80 \%$ of control values in all 3 regions (Figure 5.3). A similar small deficit was observed when assays were conducted ten weeks following the last self-administration session. The ANOVA results for 5HT levels in the frontal cortex showed an effect of MDMA intake $(F(1,33)=6.7, p<0.05)$, but no effect of abstinence or interaction between the 2 factors. In the striatum, there was also an effect of MDMA-intake $(\mathrm{F}(1,34)=5.4, \mathrm{P}<0.05)$, but no effect of abstinence or interaction. Once again the one-way ANOVAs failed to reveal a difference in 5HT levels between the 2 groups at 2 weeks or 10 weeks withdrawal. There was no significant effect of MDMA-intake, abstinence or interaction on 5HT levels in the hippocampus. 
Frontal Cortex

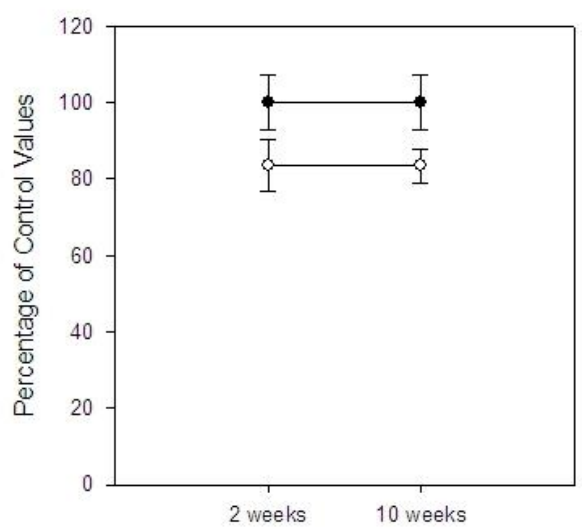

Hippocampus

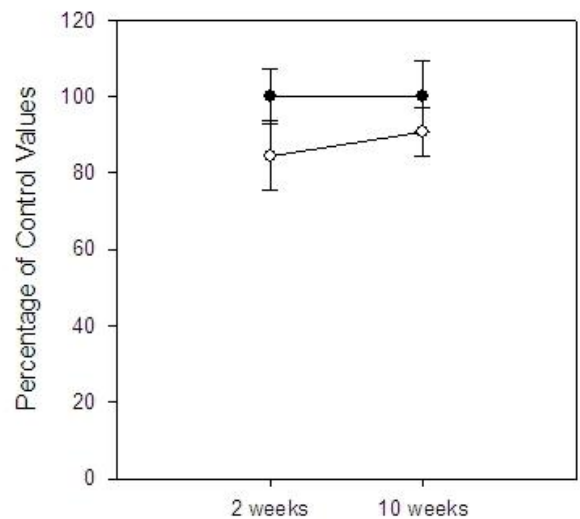

- Contro MDMA

Figure 5.3 Percentage $( \pm$ SEM) of baseline 5 HT tissue levels in brain regions of control $(n=15)$ and low dose MDMA $(n=20)$ self-administration groups.

Following the higher dose protocol, 5HT concentrations were more substantially reduced (30-35\%) in all three regions 2 weeks after the last self-administration session (Figure 5.4). A two-way ANOVA on 5HT levels revealed an effect of MDMA intake $(\mathrm{F}(1,25)=16.6, \mathrm{P}<0.05)$, abstinence $(\mathrm{F}(1.25)=14.0, \mathrm{P}<0.05)$ and an interaction between the factors $(\mathrm{F}(1,25)=14.0$, $\mathrm{p}<0.05)$ in the frontal cortex. 5HT levels in the frontal cortex were significantly decreased 2 weeks $(\mathrm{F}(1,13)=27.6, \mathrm{P}<0.05)$, but not 10 weeks following MDMA self-administration. There was no effect of MDMA intake on 5HT levels in the striatum but a significant effect of abstinence $(F(1,25)=9.9, P<0.05)$ and a significant interaction $(F(1,25)=9.9, p<0.05)$. 
There was a significant reduction in 5HT levels 2 weeks $(F(1,13)=14.2$, $\mathrm{P}<0.05)$ but not 10 weeks following MDMA self-administration. In the hippocampus, an effect of MDMA intake $(\mathrm{F}(1,25)=4.3, \mathrm{P}<0.05)$ was found, but there was no effect of abstinence or interaction between the 2 factors.

Once again, the decrease in 5HT levels produced 2 weeks following the last self-administration session $(\mathrm{F}(1,13)=5.6, \mathrm{P}<0.05)$ did not persist for the 10 week withdrawal period.
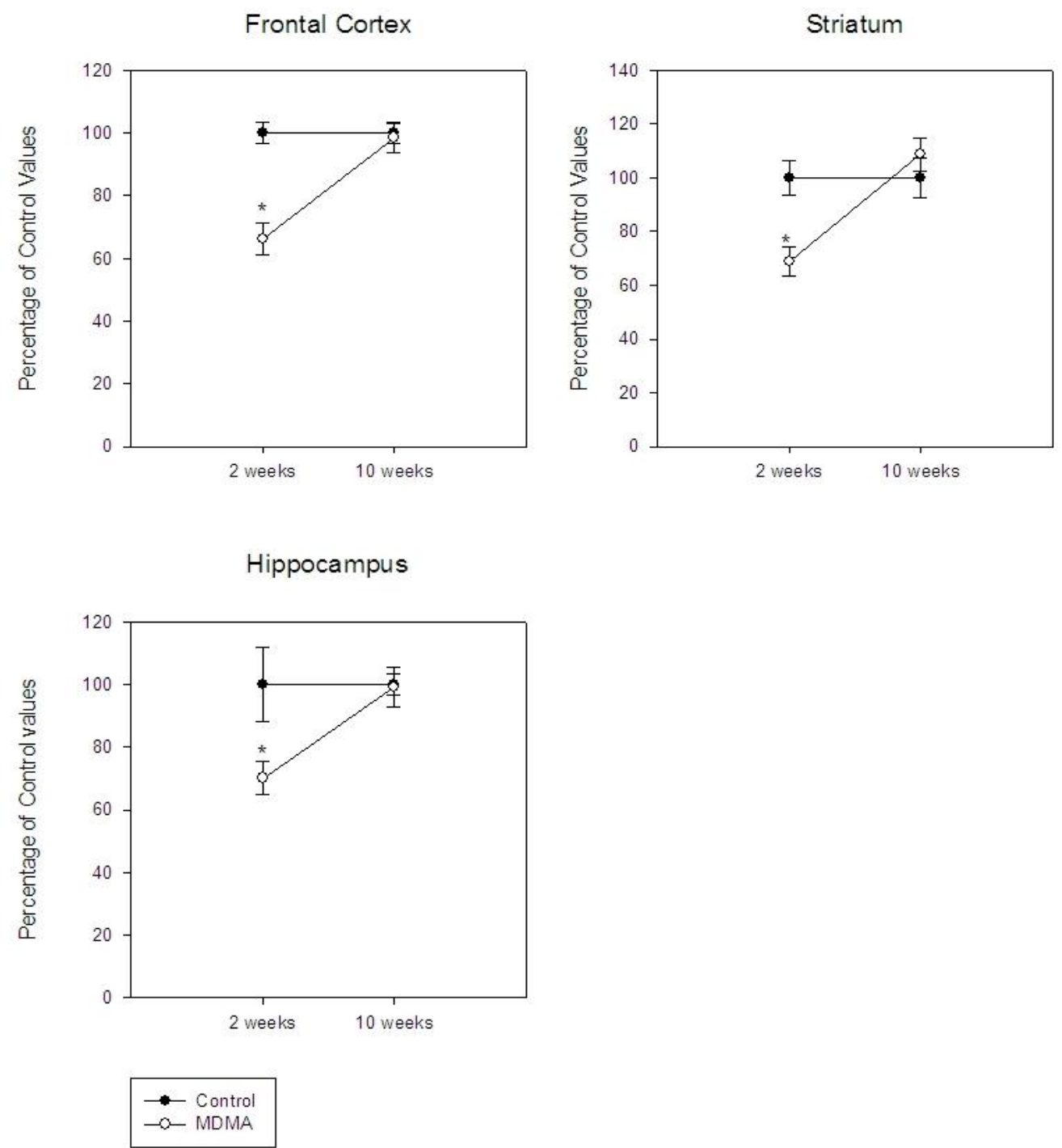

Figure 5.4 Percentage $( \pm$ SEM) of baseline 5HT tissue levels in brain regions of control ( $\mathrm{n}=14)$ and high dose MDMA (n=14) self-administration groups. ${ }^{*} \mathrm{p}<0.05$ 


\section{Discussion}

These findings demonstrate, for the first time, that self-administered MDMA produces deficits in tissue levels of 5HT that are dependent on both exposure level and withdrawal time. Following a total exposure of 165.0 mg/kg MDMA, 5HT was decreased 5 days (Schenk et al, 2011), 2 weeks and 10 weeks (Do \& Schenk, 2012; current findings) following the last selfadministration session. The higher cumulative dose of $365.0 \mathrm{mg} / \mathrm{kg}$ produced a deficit in tissue levels of 5HT 2, but not 10 weeks following the last self-administration session.

Tissue levels of 5HT following 18 months of MDMA selfadministration $(120.0 \mathrm{mg} / \mathrm{kg}-250.0 \mathrm{mg} / \mathrm{kg})$ in monkeys, however, were not decreased in a similar manner (Fantegrossi et al, 2004) even though, the total intake was substantially greater than experimenter-administered doses that produced robust reductions of tissue 5HT (Ricaurte et al, 1988). The failure might be due to the relatively low daily self-administered MDMA.

The decrease in tissue levels of 5HT in the present study was substantially smaller than 5HT depletions produced by similar, and in some instances smaller, doses of experimenter-administered MDMA (Scanzello et al, 1996; Sabol et al, 1993). The total intake of MDMA over the course of self-administration in the low dose group was comparable to that in experimenter-administered studies (twice daily injections of $20.0 \mathrm{mg} / \mathrm{kg}$ over 4 consecutive days) in which robust depletions of 5HT (70-80\%) have been observed 2 weeks following exposure (Battaglia et al, 1988; Commins et al, 1987; Sabol et al, 1996). Although the high dose group selfadministered more than twice the amount of MDMA, the decrease in tissue levels of 5HT was still relatively small (30-35\%). The average daily intake of MDMA over the last 3 days of self-administration was $15.8 \pm 1.2 \mathrm{mg} / \mathrm{kg}$ in the high dose group. This is substantially less than majority of dosing regimens administered by the experimenter in which the average daily dose is about $40.0 \mathrm{mg} / \mathrm{kg}$ (Commins et al, 1988; Battaglia et al, 1988; Sabol et al, 1996; Scanzello et al, 1993). It is possible that the smaller deficit of tissue 5HT levels observed 2 weeks after MDMA self-administration was due to the lower average daily intake. 
Alternatively, pre-exposure to MDMA during self-administration might have provided neuroprotection against MDMA-induced depletions (Bhide et al, 2009; Piper et al, 2010). An intermittent dosing regimen of MDMA administered during a pre-treatment phase of 25 days prevented further reductions in tissue levels of 5HT following a "binge" dose of MDMA (Piper et al, 2010). Pre-treatment with daily injections of MDMA $(10.0 \mathrm{mg} / \mathrm{kg})$ also prevented subsequent MDMA-produced 5HT deficits in the frontal cortex, striatum and hippocampus (Bhide et al, 2010). This neuroprotection was relatively transient in nature and was attenuated when the interval between the preconditioning regimen and the 5HT-depleting regimen was extended from 1 day to 4 days. Furthermore, the magnitude of neuroprotection was greater with greater exposure (Bhide et al, 2009). A similar form of neuroprotection might have occurred as a result of low levels of MDMA self-administration.

These findings suggest that NOR deficits may have been due to decreased tissue levels of 5HT 2 weeks following the low dose of MDMA self-administration. NOR deficits, however, recovered 10 weeks following MDMA self-administration, when tissue levels of 5HT were decreased (current findings). This was unexpected as previous reports have suggested that deficits in tissue levels of 5HT may result in impaired NOR performance in rats (King et al, 2009; Jenkins et al, 2010; Lieben et al, 2005). For example, chronic tryptophan depletion decreased 5HT levels in the frontal cortex, striatum and hippocampus 21 days following treatment, and this was accompanied by deficits in the NOR task (Jenkins et al, 2010). Depletion of tissue levels of 5HT, following 5,7-DHT (5,7Dihydroxytryptamine) lesions, also impaired NOR performance in rats (King et al, 2009; Lieben et al, 2005).

Regardless, MDMA self-administration produced deficits in tissue levels of 5HT that were both dose-dependent and recovered over time. The recovery of these deficits further supports the notion that the underlying mechanisms are neuroadaptive in nature rather than produced by loss of 5HT neurons. The possibility that MDAM-produced deficits can be reversed by targeting synthesis of $5 \mathrm{HT}$ is examined in the subsequent sections. 


\title{
Chapter 6: Mechanisms of 5HT-produced deficits
}

\author{
6.1: The role of the 5HT1a autoreceptor: 8-OH-DPAT-induced lower lip \\ retraction
}

Overview

MDMA-produced deficits in tissue levels of 5HT might be due to decreased 5HT synthesis, induced as a result of a supersensitive $5 \mathrm{HT}_{1 \mathrm{a}}$ autoreceptor. Lower lip retraction (LLR) in rats has been attributed to the response of $5 \mathrm{HT}_{1 \mathrm{a}}$ autoreceptor (see introduction). Therefore, the LLR paradigm was used to assess the sensitivity of the $5 \mathrm{HT}_{1 \mathrm{a}}$ autoreceptor following both experimenter and self-administered MDMA. Rats received MDMA (or vehicle) treatment via self-administration or the experimenteradministered regimen. For the self-administration group, a total intake of $315.0 \mathrm{mg} / \mathrm{kg}$ was chosen and the experimenter-administered MDMA group received 4 injections of MDMA (i.p. in a $0.9 \%$ saline vehicle at $10.0 \mathrm{mg} / \mathrm{ml}$ ) administered every 2 hours. For both groups testing was conducted 2 weeks following MDMA exposure. The parameters of these two dosing regimens were chosen as they had been found to produce deficits in tissue levels of 5HT in rats 2 weeks following MDMA exposure (See Chapter 5 and Scanzello et al, 1993). Controls were tested 2 weeks following administration of vehicle either using the self- or experimenter-administered protocol. 
Results

Self-administration

Of the original cohort $(n=24), 46 \%$ completed the criterion and the average number of days required to self-administer 90 infusions of 1.0 $\mathrm{mg} / \mathrm{kg}$ MDMA was $12 \pm 1.1$ days. The distribution was positively skewed and extended testing (>10 days) was generally required to meet the criterion (Figure 6.1). Once this criterion was met, an average of $15.6 \pm 2.5$ days was required to produce the subsequent 450 lever presses.

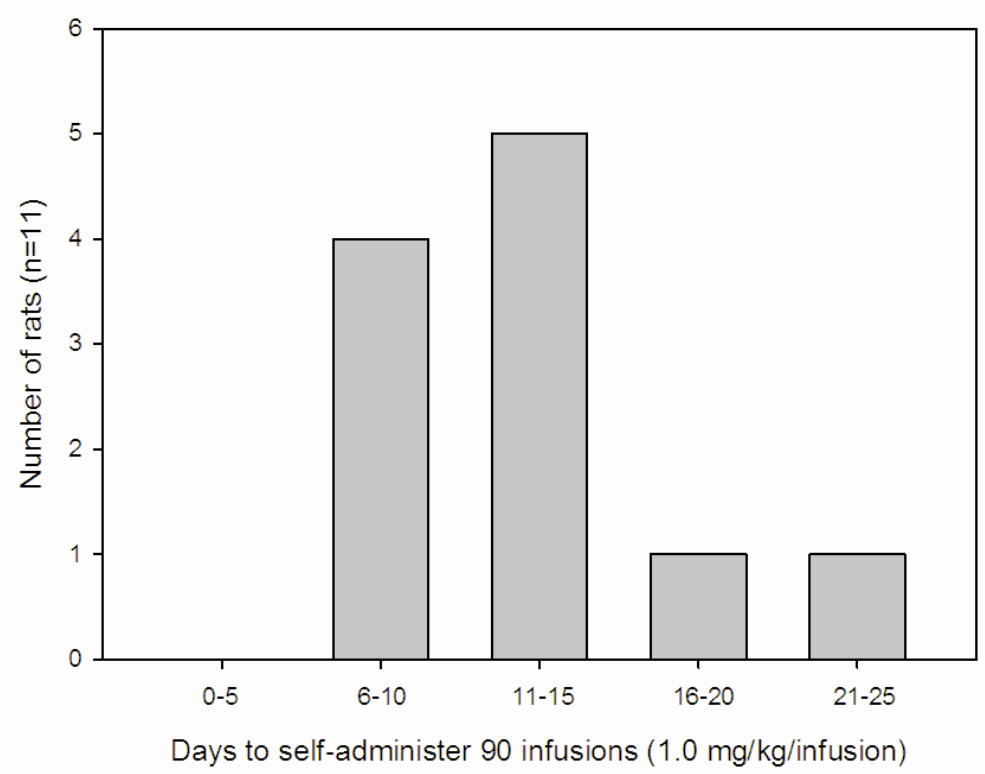

Figure 6.1 Frequency distribution of number of days required t selfadminister 90 infusions of $1.0 \mathrm{mg} / \mathrm{kg}$ MDMA

Rats self-administered relatively low levels of MDMA (5.8 \pm 1.6 $\mathrm{mg} / \mathrm{kg} /$ day) during the first 3 days of self-administration (Figure 6.2). During the last 3 days of self-administration of the $1.0 \mathrm{mg} / \mathrm{kg} / \mathrm{infusion}$ dose, average daily intake increased to $13 \pm 0.9 \mathrm{mg} / \mathrm{kg}(\mathrm{t}(20)=3.9, * \mathrm{p}<0.05)$. When the dose was reduced to $0.5 \mathrm{mg} / \mathrm{kg} /$ infusion dose, average daily intake over the first 3 days was $10.2 \pm 1.4 \mathrm{mg} / \mathrm{kg}$ and this increased to 23.6 
$\pm 3.4 \mathrm{mg} / \mathrm{kg}\left(\mathrm{t}(20)=3.63,{ }^{*} \mathrm{p}<0.05\right)$ during the last 3 days of selfadministration (Figure 6.2). A total dose of $313.9 \pm 9.5 \mathrm{mg} / \mathrm{kg}$ was selfadministered.
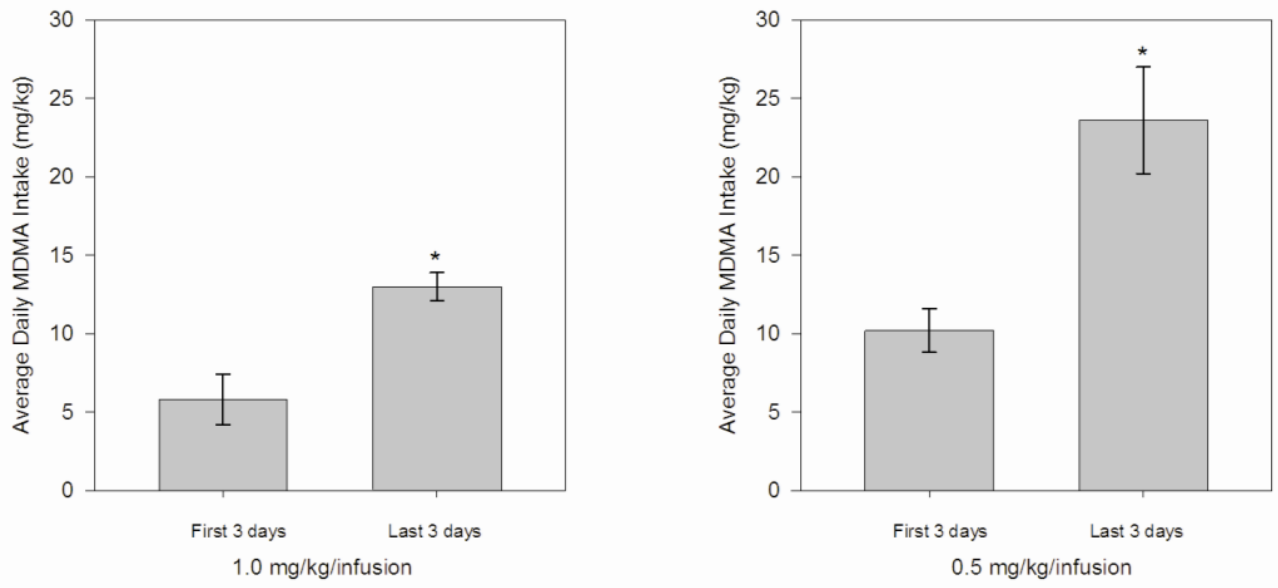

Figure 6.2 Average daily intake ( \pm SEM) during the first and last 3 days of self-administration of $1.0 \mathrm{mg} / \mathrm{kg} / \mathrm{infusion}$ and $0.5 \mathrm{mg} / \mathrm{kg} / \mathrm{infusion}$ doses, $* \mathrm{p}<0.05$

\section{Lower Lip Retraction}

Figure 6.3 shows the total lower lip retraction score, during 60 minutes of testing, as a function of 8-OHDPAT dose following MDMA or saline self-administration. The score increased with increasing dose $(\mathrm{F}(4,60)=84.992, \mathrm{p}<0.05)$ but there was no difference in the response as a function of pre-treatment. Other groups received experimenter-administered MDMA 2 weeks prior to the lower lip retraction test with similar results (Figure 6.4). There was also an effect of 8-OHDPAT dose $(F(4,61)=82.65$, $\mathrm{p}<0.05)$ but no effect of pre-treatment. 


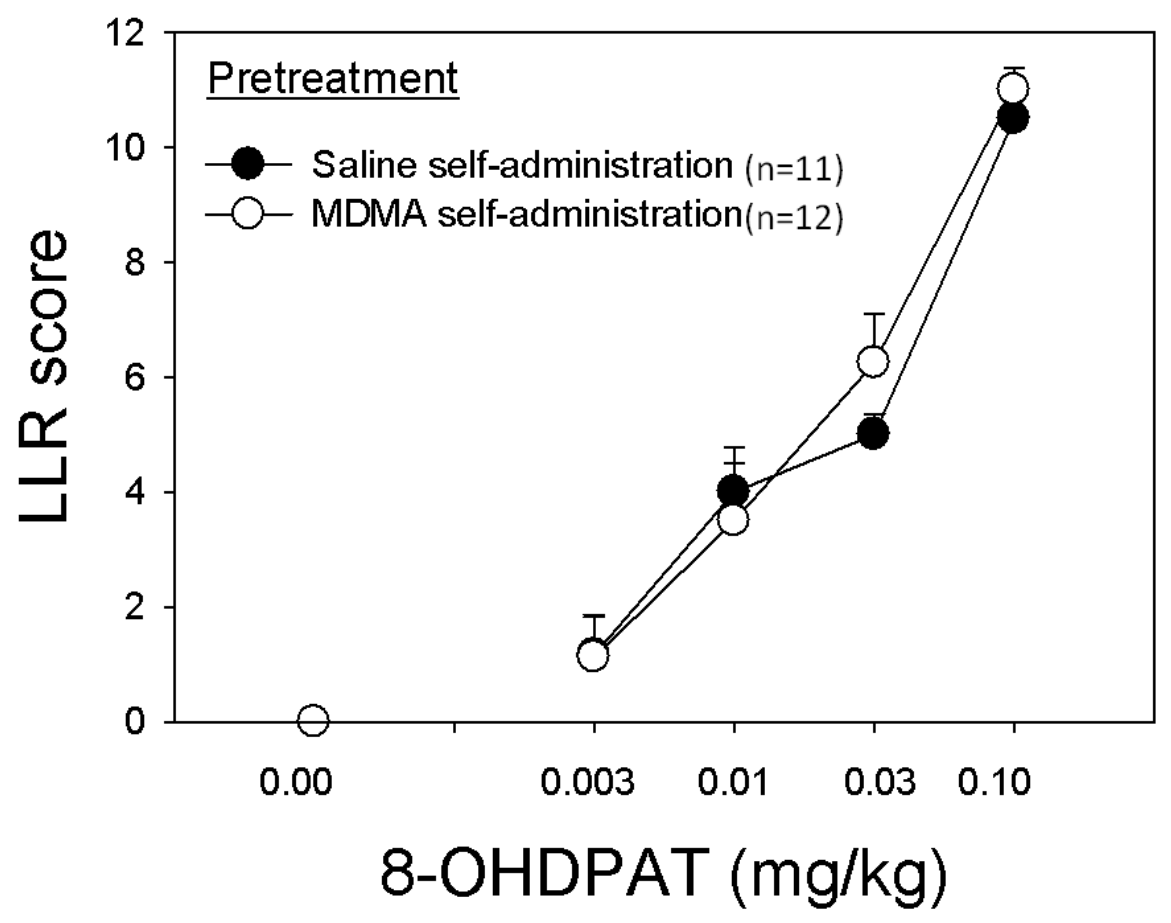

Figure 6.3 Dose-response curve for LLR as a function of MDMA selfadministration VS controls. 


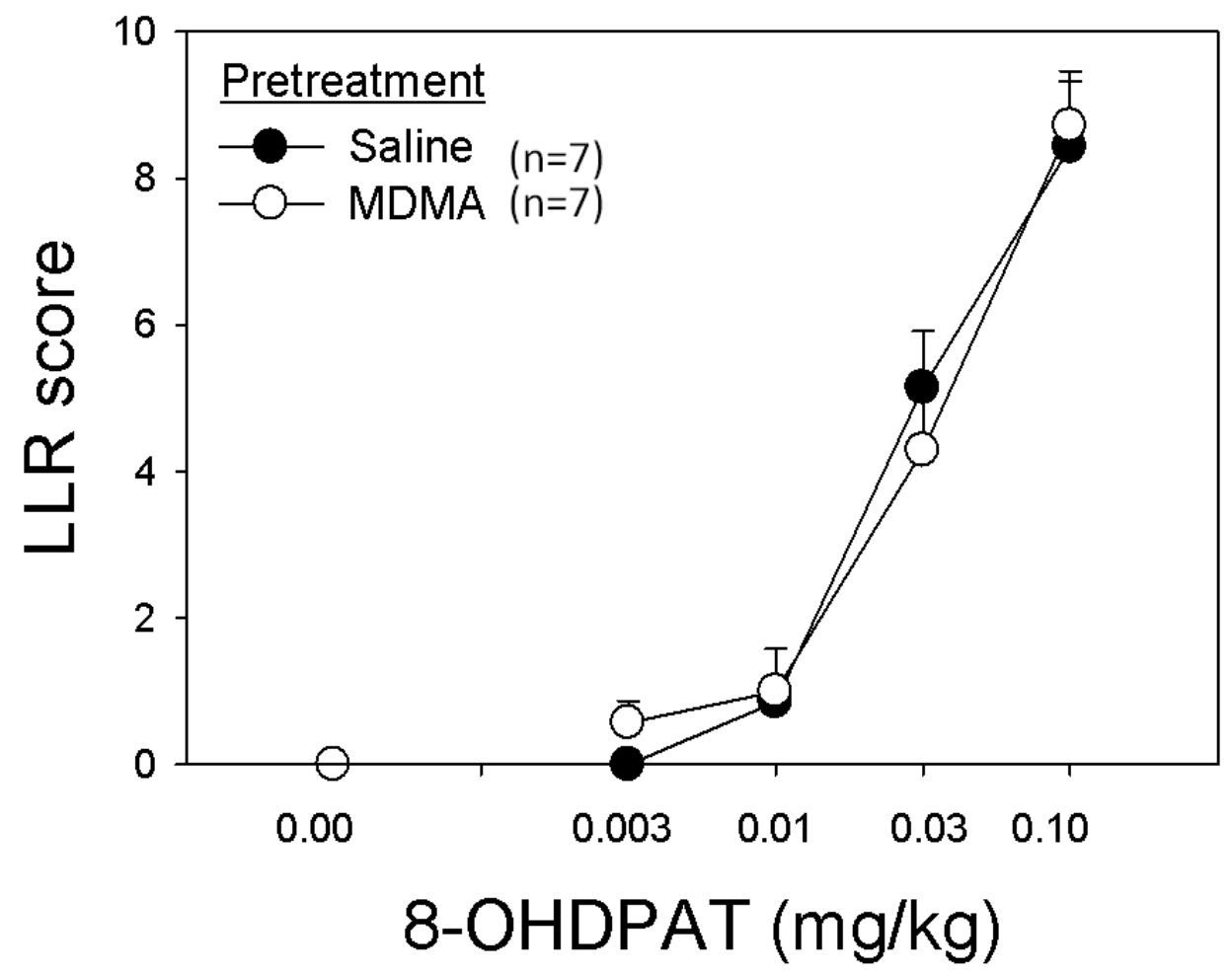

Figure 6.4 Dose-response curve for LLR as a function MDMA treatment VS controls. 
6.2: Effects of chronic 8-OH-DPAT on tissue levels of 5HT following MDMA treatment

Overview

If a supersensitivity of the $5 \mathrm{HT}_{1 \mathrm{a}}$ autoreceptor underpins MDMAproduced 5HT deficits, then desensitisation following MDMA exposure would be expected to restore the 5HT levels. To test this hypothesis, a protocol of chronic 8-OH-DPAT treatment which had been previously shown to desensitize the $5 \mathrm{HT}_{1 \mathrm{a}}$ autoreceptor was used (Kreiss \& Luki, 1992). Rats were treated with 8-OH-DPAT $(1.0 \mathrm{mg} / \mathrm{kg} /$ day, s.c., base weight) or saline for 7 days, since this procedure decreased the response of the autoreceptor (Kreiss \& Luki, 1992). Chronic 8-OH-DPAT attenuated 8OH-DPAT-induced inhibition of striatal 5HT release, measured using in vivo microdialysis (Kreiss \& Luki, 1992), suggesting a desensitisation of the autoreceptor.

Initial groups were tested to ensure adequacy of the treatment regimen. For these groups, 8-OH-DPAT-induced LLR was measured 7 days following this treatment

Rats ( $\mathrm{n}=23$ ) self-administered $315.0 \mathrm{mg} / \mathrm{kg}$ MDMA. This dose was chosen since it produced deficits in tissue levels of 5HT 2 weeks following the last self-administration session (See Chapter 5). Controls were placed in self-administration test chambers for 2 hour sessions, 6 days a week, for 4 weeks. Six days following the last in self-administration session (or last day in self-administration chamber for controls), subjects received either daily injections of 8-OH-DPAT (1.0 mg/kg base weight, s.c.) or saline for 7 days. Twenty-four hours following the last injection, brains were extracted and tissue samples from the frontal cortex, hippocampus and striatum were taken. Neurochemical assays were conducted using HPLC to determine the tissue levels of 5 HT in samples. 
Results

The ability of the 8-OH-DPAT regimen to desensitise $5 \mathrm{HT}_{1 \mathrm{a}}$ autoreceptors was verified using the LLR paradigm (Figure 6.5). A repeated measures ANOVA revealed an effect of dose $(F(1,12)=287.9), p<0.05)$, an effect of pre-treatment $(F(1,12)=6.6, p<0.05)$ and an interaction between the 2 factors $(F(1,12)=10.3, p<0.05)$. Post-hoc analysis were conducted using student's t-tests with bonferroni corrections $(\mathrm{p}<0.01)$. The LLR score was significantly different between pre-treatment groups at both the $0.03 \mathrm{mg} / \mathrm{kg}$ and $0.10 \mathrm{mg} / \mathrm{kg}(\mathrm{p}<0.01)$.

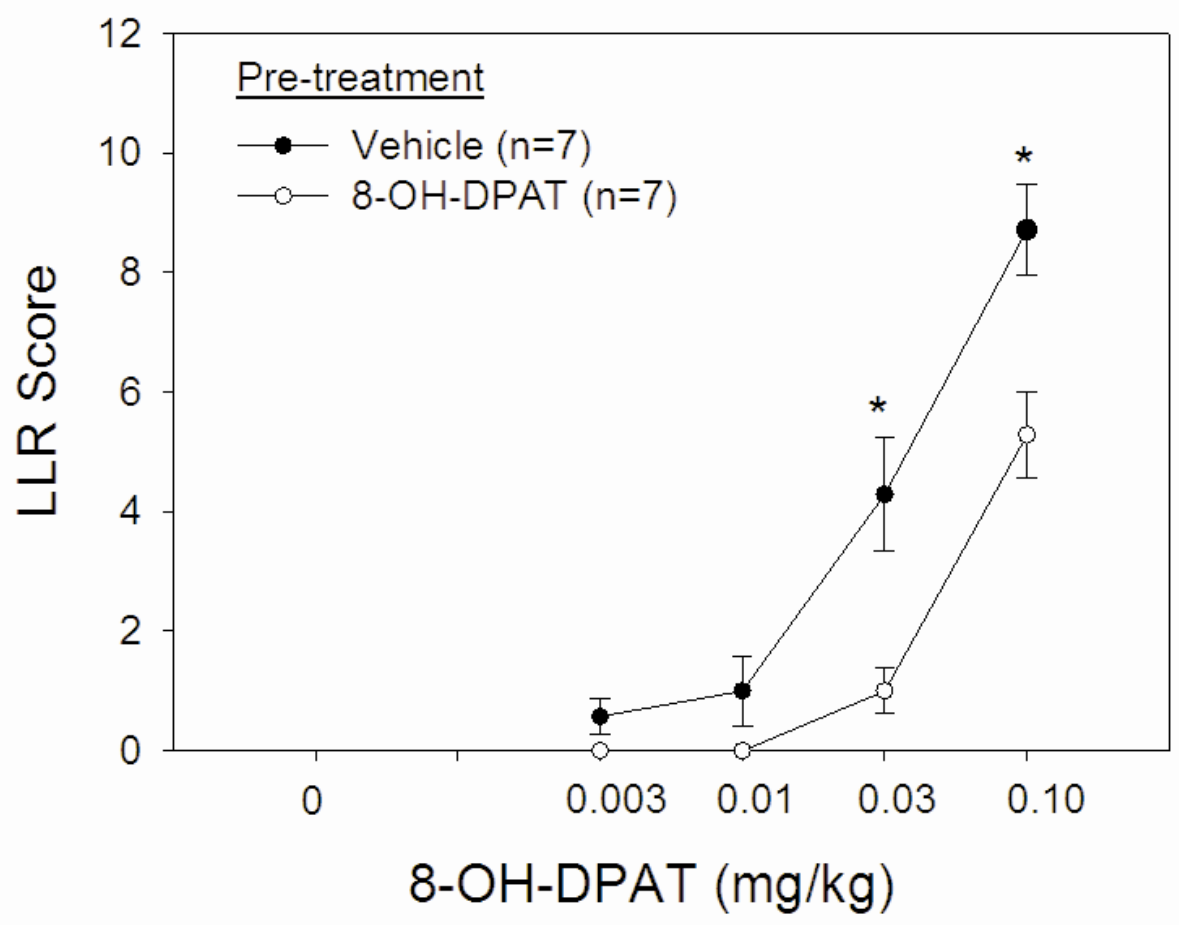

Figure 6.5 LLR as a function of 8-OH-DPAT dose, following 8-OH-DPAT or vehicle pre-treatment, $* \mathrm{p}<0.05$

Of the original cohort of rats, $40 \%$ met the criterion and extended testing was generally required (>10days; Figure 6.6). An average of $17 \pm$ 0.8 days was required to meet the initial criterion and an additional $33.2 \pm$ 1.3 days to meet the subsequent criterion of 450 lever presses on the 0.5 $\mathrm{mg} / \mathrm{kg} /$ infusion dose. 


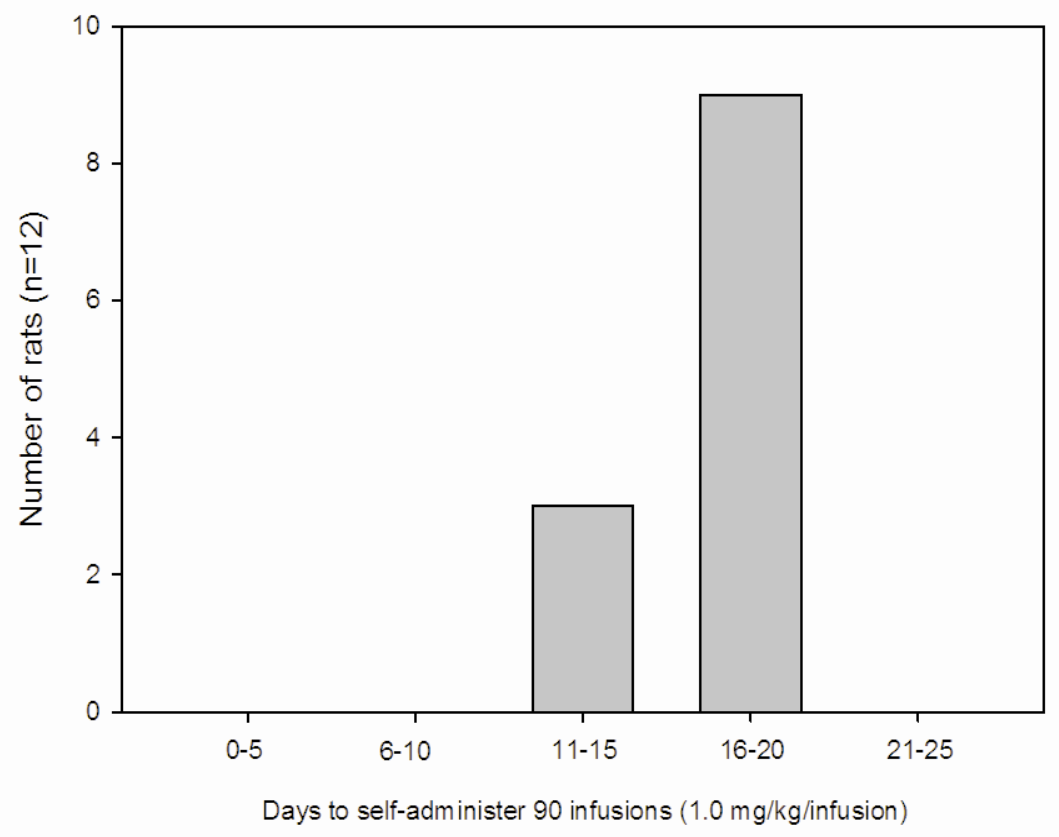

Figure 6.6 Frequency distribution of number of days required to selfadminister 90 infusion of $1.0 \mathrm{mg} / \mathrm{kg} /$ infusion

During the first 3 days of self-administration, the average daily intake was $2.9 \pm 0.6 \mathrm{mg} / \mathrm{kg}$ and this increased to $11.3 \pm 0.9 \mathrm{mg} / \mathrm{kg}$ during the last 3 days of self-administration of $1.0 \mathrm{mg} / \mathrm{kg} /$ infusion $(\mathrm{t}(22)=7.67$, $\mathrm{p}<0.05$; Figure 6.7). When the dose was decreased to $0.5 \mathrm{mg} / \mathrm{kg} / \mathrm{infusion}$, the average daily intake over the first and last 3 days of self-administration was $12.3 \pm 1.1 \mathrm{mg} / \mathrm{kg}$ and $16.7 \pm 0.9 \mathrm{mg} / \mathrm{kg},(\mathrm{t}(22)=3.01, \mathrm{p}<0.05)$ respectively. 

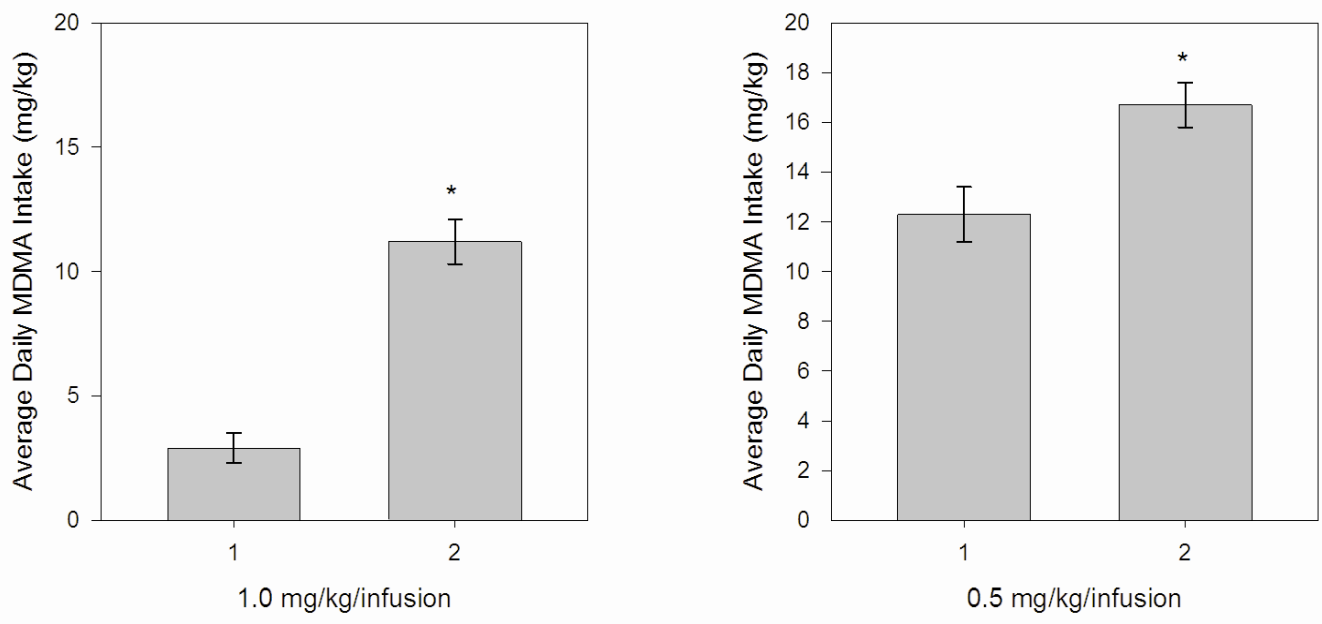

Figure 6.7 Average daily intake $( \pm$ SEM) during the first and last 3 days of self-administration of $1.0 \mathrm{mg} / \mathrm{kg} /$ infusion and $0.5 \mathrm{mg} / \mathrm{kg} /$ infusion doses. $* \mathrm{p}<0.05$

A two-way ANOVA revealed that there was an effect of MDMA on tissue levels of 5HT in all three brain regions (frontal cortex, $\mathrm{F}(3,23)=19.8$, $\mathrm{p}<0.05)$; striatum, $\mathrm{F}(3,23)=16.3, \mathrm{p}<0.05$; hippocampus, $\mathrm{F}(3,23)=11.9$, $\mathrm{p}<0.05$; Figure 6.8). MDMA pre-treatment reduced tissue levels of 5HT by approximately $25 \%$. However, there was no effect of the 8-OH-DPAT treatment and no interaction between 8-OH-DPAT treatment and MDMA exposure. 
Frontal Cortex

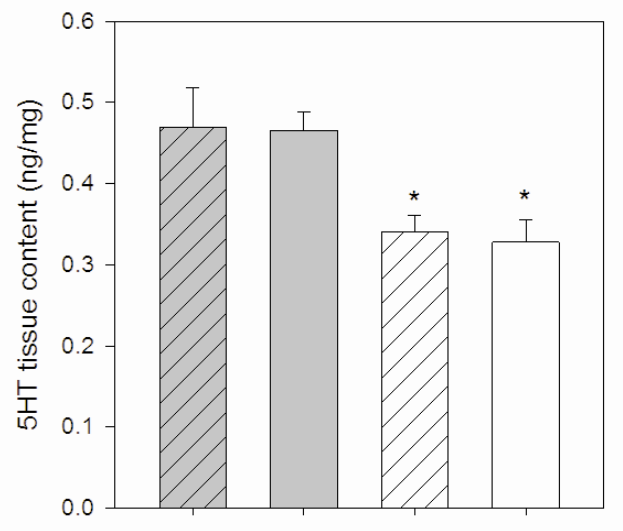

Hippocampus

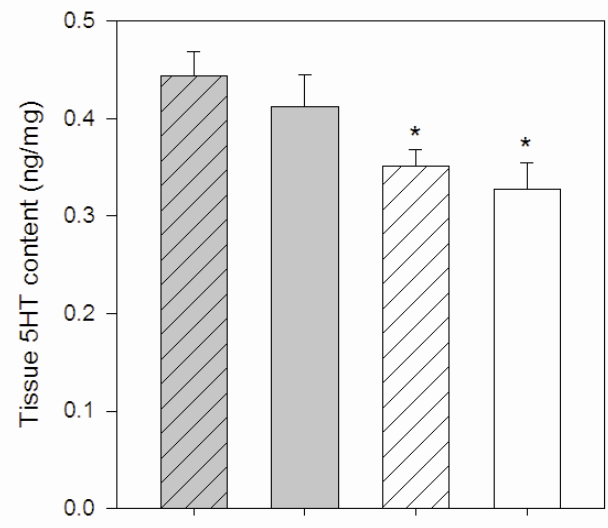

Striatum

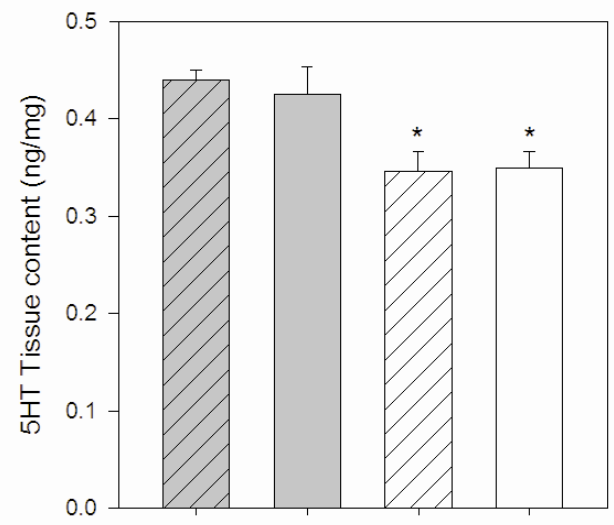

Figure 6.8 Tissue $5 \mathrm{HT}$ concentration $(\mathrm{ng} / \mathrm{mg})$ from the 4 different treatment groups * $\mathrm{P}<0.01$, student's t-test, compared to relevant control group 


\section{3: Effects of chronic trazodone treatment following MDMA exposure}

\section{Overview}

Trazodone is one of the most commonly prescribed treatments for depression (Mendelson et al, 2005; Nierenberg et al, 1994). It enhances extracellular 5HT levels as an agonist/antagonist and a 5HT reuptake inhibitor (Mittur, 2011; Maj et al, 1979; Pazzagli et al, 1999; Raffa et al, 1992; Hingtgen et al, 1984). Trazodone also affects both the NE and DA system, although its long-term effects appear to be mainly dominated by enhancement of serotonergic neurotransmission (Egashira et al, 1999). The exact mechanism by which trazodone confers its effects, either acutely or in the long-term is not entirely clear.

Evidence suggests, however, that sustained administration of trazadone desensitizes the 5HT1a autoreceptor, and this may be the mechanisms by which trazadone enhances serotonergic transmission (Ghanbari et al, 2010). In the current experiment, the effect of a treatment regimen of trazadone that has been shown to desensitize the 5HT1a autoreceptor, was used to determine effects of MDMA. Trazodone was administered continuously via subcutaneously implanted osmotic minipumps according to the protocol described in Ghanbari et al (2010).

\section{Minipump procedure}

Two days following MDMA exposure (4 X $10.0 \mathrm{mg} / \mathrm{kg}$ MDMA, every 2 hours), osmotic Alzet minipumps (model: 2ML2; Alza, Palo Alto, CA), were implanted subcutaneously under the skin in the mid-scapular regions under anesthesia produced by an injection (i.p.) of ketamine $(90.0 \mathrm{mg} / \mathrm{kg})$ and xylazine $(5.0 \mathrm{mg} / \mathrm{kg})$. Surgery was carried out using aseptic 
techniques on a warming pad and eyes were lubricated with vasoline to prevent drying out. Following surgery, rats received post-operation care which included electrolytes $(20 \mathrm{ml}, \mathrm{s.c})$ and Carprofen $(5.0 \mathrm{mg} / \mathrm{kg}$, s.c., also administered 24 and $48 \mathrm{hrs}$ following surgery). The pump remained in place for 14 days, after which, brains were extracted and tissue samples from the frontal cortex, striatum and hippocampus were obtained. Following brain tissue extraction, minipumps were removed and residual volumes were measured to ensure that the minipumps had been functioning properly.

Results

Sixteen days following MDMA treatment, 5HT levels were $60 \%$ of control values in the frontal cortex and $65 \%$ of control values in the hippocampus. Striatal 5HT levels were comparable to control values (97\%; Figure 6.9). A two-way ANOVA showed an effect of pre-treatment $(F(1,19)=9.72, p<0.05)$, but no effect of minipump treatment and no interaction between the two factors. Similarly, in the hippocampus, the twoway ANOVA revealed an effect of pre-treatment $(\mathrm{F}(1,19)=1.002, \mathrm{p}(0.05)$, but no effect of minipump treatment or interaction. There was no significant effect of pre-treatment or minipump treatment in the hippocampus. 
Frontal Cortex

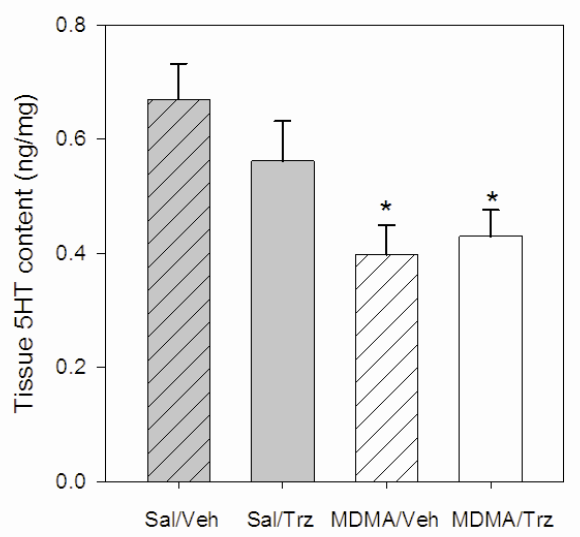

Hippocampus

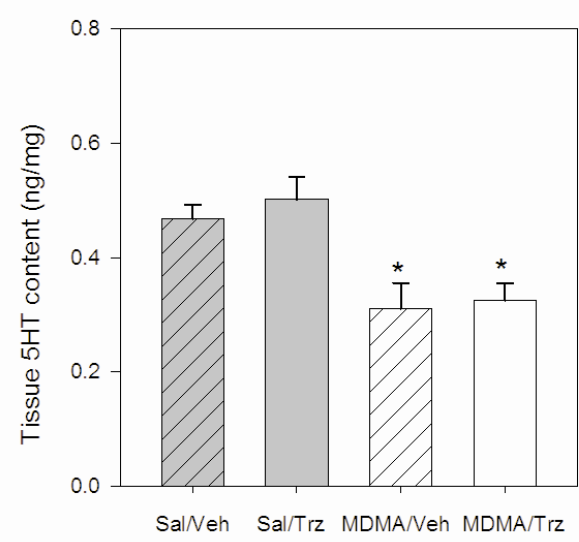

Striatum
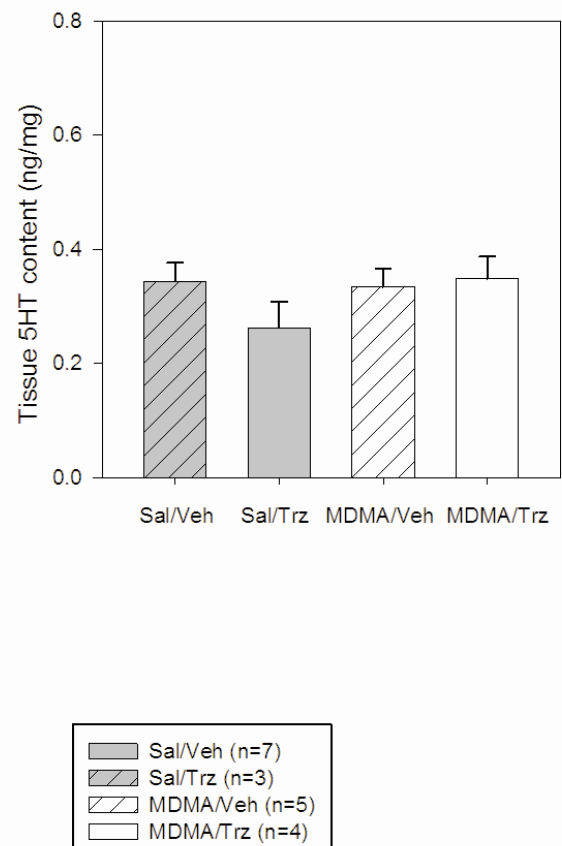

Figure 6.9 Regional brain tissue 5HT content $(\mathrm{ng} / \mathrm{mg})$ of rats from the 4 different treatment groups, ${ }^{*} \mathrm{p}<0.05$ compared to relevant control groups

In the frontal cortex, a two-way ANOVA on 5HIAA levels revealed an effect of pre-treatment $(F(1,19)=18.1, p<0.05)$, an effect of minipump treatment $(\mathrm{F}(1,19)=4.48, \mathrm{p}<0.05)$ and an interaction between the two factors $(F(1,19)=4.8, p<0.05)$. A post-hoc one-way ANOVA showed that the trazodone reduced 5HIAA levels in rats that received a saline pre-treatment $(F(1,9)=8.5, p<0.05)$ but not in those treated with MDMA. A two-way ANOVA revealed no effect of pre-treatment or minipump and no interaction between the two factors for striatal 5HIAA levels. The main effect of minipump treatment approached significance $(F(1,19)=4.07$, $\mathrm{p}=0.062$ ). In the hippocampus, the two-way ANOVA showed that there was 
an effect of pre- treatment $(F(1,19)=28.8, p<0.05)$ but no effect of minipump treatment and no interaction. In rats that received a vehicle treatment in the minipumps, 5HIAA levels were reduced by MDMA $(\mathrm{F}(1,11)=28.9, \mathrm{p}<0.05)$. Trazodone treatment failed to alter the MDMAproduced decrease in 5HIAA levels $(\mathrm{F}(1,6)=7.2, \mathrm{p}<0.05)$. These data suggest that trazodone reduced 5HIAA levels in both the frontal cortex and hippocampus and there was a trend for this decrease in the striatum (Figure 6.10). Because there was no consistent effect of trazodone treatment on 5HT or 5HIAA levels in control animals, the treatment might have been ineffective.
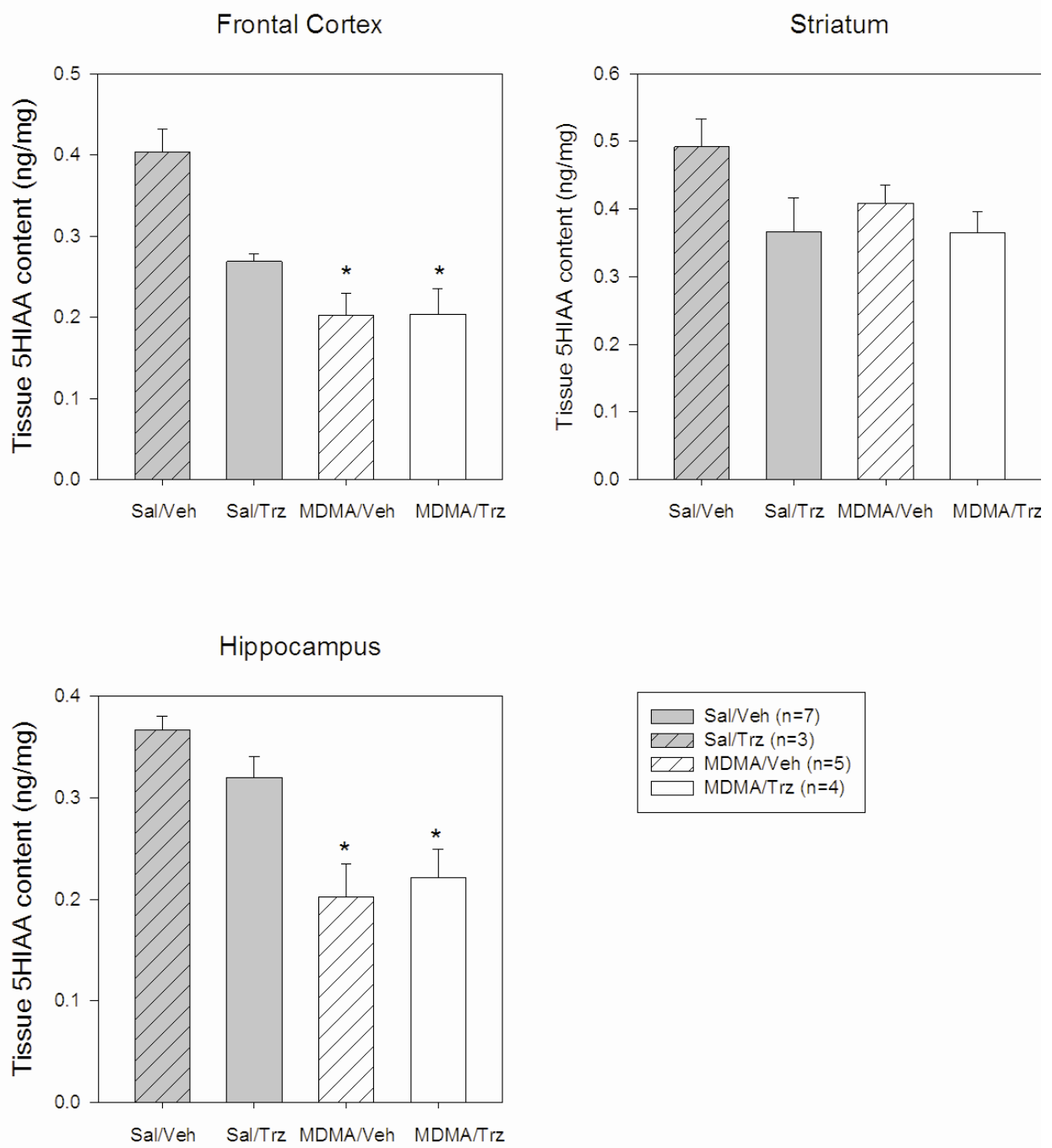

Figure 6.10 Regional brain tissue 5HIAA content (ng/mg) from the 4 different treatment groups, ${ }^{*} \mathrm{p}<0.05$ compared to $\mathrm{Sal} / \mathrm{Veh}$ 


\section{Discussion}

The current experiments were designed to test the hypothesis that 5HT deficits resulting from MDMA are due to a supersensitisation of $5 \mathrm{HT}_{1 \mathrm{a}}$ autoreceptor. MDMA exposure failed to alter the dose-response curve for 8OH-DPAT-induced LLR and a regimen of 8-OH-DPAT which desensitized the $5 \mathrm{HT}_{1 \mathrm{a}}$ autoreceptor failed to reverse the $5 \mathrm{HT}$ deficits in produced by MDMA exposure. These findings suggest that the $5 \mathrm{HT}_{1 \mathrm{a}}$ autoreceptor supersensitisation is not the mechanism for $5 \mathrm{HT}$ deficits.

In order to further investigate the role of the $5 \mathrm{HT}_{1 \mathrm{a}}$ autoreceptor, the effect of chronic trazodone treatment on the MDMA-produced decrease in 5HT was tested. In this study, MDMA treatment reduced 5HT in the frontal cortex and hippocampus, but striatal levels were unchanged. This dosing regimen produced $5 \mathrm{HT}$ deficits in all three brain regions when assays were conducted 14 days later (unpublished data). The striatum had been previously shown to be more resistant to the effects of MDMA (Lehmann et al, 1992; O'Shea et al, 2006; Sabol et al, 1996) and the current data suggest that striatal 5HT levels recovered by 18 days.

In the frontal cortex and hippocampus, chronic treatment with trazodone was ineffective in reversing or attenuating the MDMA-produced decrease in 5HT levels. Trazodone alone, however, had no effect on 5HT levels in rats treated with vehicle. 5HIAA levels were decreased in the frontal cortex suggesting that, at least in some regions, the treatment altered 5HT metabolism or utilization (Thompson et al, 2004). This restricted effect, however, suggests that a more stringent exposure regimen might be required.

The effects of trazodone on the 5HT system may not have been sufficient to override the effect of MDMA treatment. Of interest, the acute effects of fluoxetine were attenuated in rats by pre-treatment with MDMA (Ando et al, 2006; Durkin et al, 2008). This attenuation was observed despite recovery of SERT. This study suggests that long-lasting alterations in the 5HT system blunted the effects of fluoxetine, and this could also explain the ineffectiveness of trazodone treatment in the current study. 
Taken together, the current findings are not consistent with the idea that MDMA exposure alters the response of the $5 \mathrm{HT}_{1 \mathrm{a}}$ autoreceptor (Agguire et al, 1995). In particular, it has been suggested that desensitisation as compensatory mechanism, functioned to increase 5HT synthesis following MDMA (Agguire et al, 1995). An alternate explanation is that the decrease in 8-OH-DPAT binding in the dorsal raphe following MDMA reflected down-regulation of $5 \mathrm{HT}_{1 \mathrm{a}}$ heteroceptors, which are highly localised to this region.

These experiments fail to support a role of $5 \mathrm{HT}_{1 \mathrm{a}}$ autoreceptors in MDMA-produced 5HT deficits or the use of pharmacological manipulations to reverse the 5HT deficits. Rather, the 5HT deficits produced by MDMA might reflect the well-documented decrease in TPH activity (Stone et al, 1986; 1987; Schmidt \& Taylor, 1987; 1988). 


\section{Chapter 7: The role of TPH in MDMA-produced 5HT deficits: Effects of tryptophan loading}

\section{$\underline{\text { Overview }}$}

Because chronic tryptophan treatment increases tissue levels of 5HT by increasing TPH activity, it was hypothesised that it might facilitate recovery of tissue 5HT levels following MDMA exposure. This idea was tested in the current experiment using a treatment of chronic tryptophan administration which had previously been shown to increase 5HT levels (Sanchez et al, 2008).

Subjects were pre-treated with MDMA (See Chapter 6) and 7 days following exposure, were administered L-tryptophan $(125.0 \mathrm{mg} /$ day in saline $(0.9 \%)$ vehicle) or vehicle via oral gavage for 7 consecutive days. Twenty four hours following the last day of tryptophan loading, brains were extracted and the frontal cortex, striatum and hippocampus were dissected. Neurochemical assays were conducted and 5HT levels were measured.

\section{$\underline{\text { Results }}$}

A two-way ANOVA revealed that there was an effect of MDMA in all three brain regions examined $(\mathrm{FC}: \mathrm{F}(1,25)=22.0$, ST: $\mathrm{F}(1,24)=7.03$, Hip: $\mathrm{F}(1,24)=8.0, \mathrm{p}<0.05)$, but no effect of the L-tryptophan or interaction. Tryptophan treatment itself also failed to produce changes in 5HT levels of control rats. 


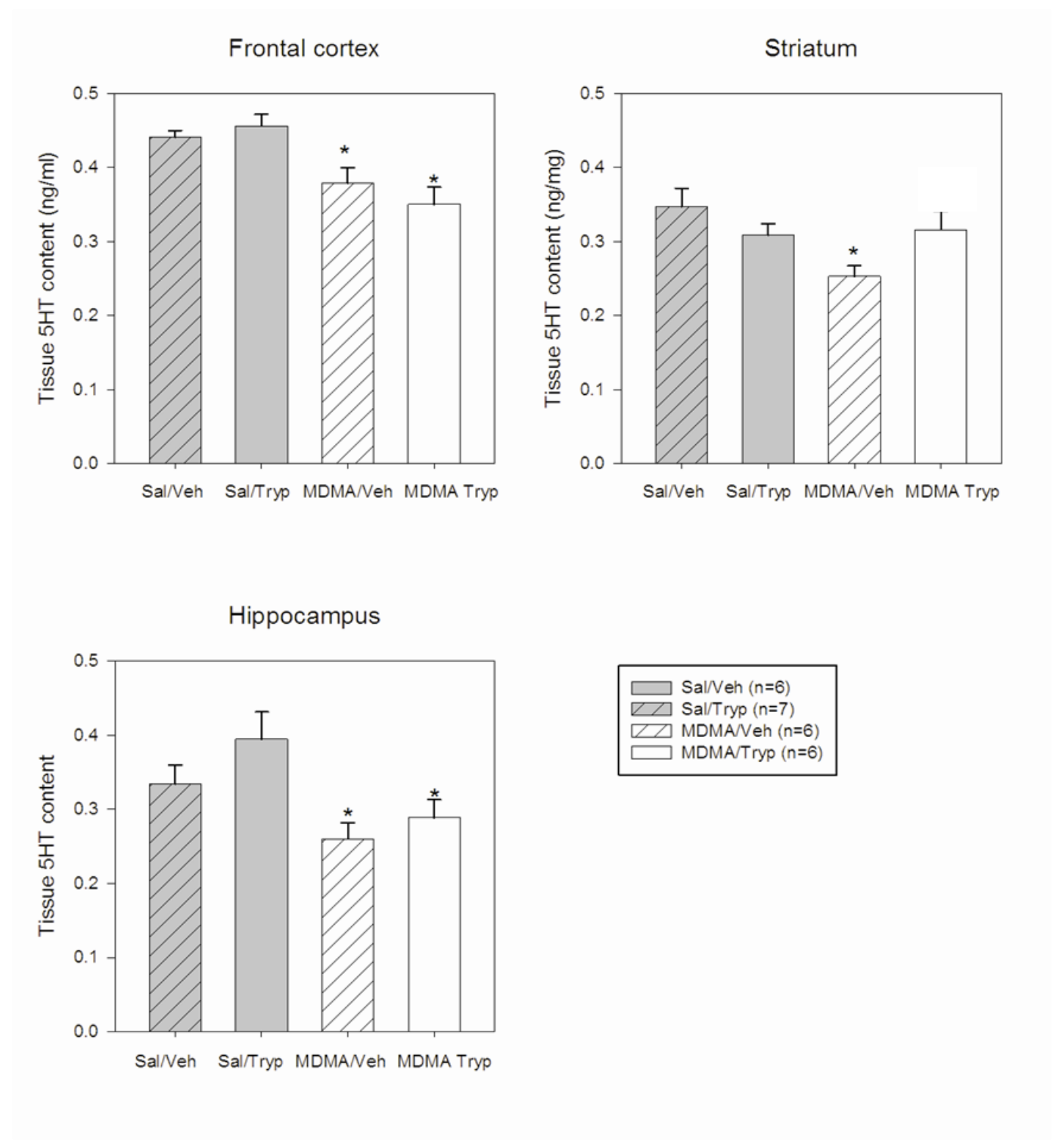

Figure 7.1 Regional brain tissue 5HT levels of rats from 4 different treatment groups, ${ }^{*} \mathrm{p}<0.05$ compared to relevant control groups.

\section{$\underline{\text { Discussion }}$}

Tryptophan loading failed to alter tissue levels of 5HT in either MDMA or saline-treated rats, despite previous reports (Sanchez et al, 2008). It is possible that the difference in times of administration influenced the effectiveness of the treatment since Sanchez et al (2008) administered tryptophan at $7 \mathrm{pm}$ while in the current study, gavaging took place between 4 and $5 \mathrm{pm}$ daily. It is hard to imagine, however, that this $2 \mathrm{hrs}$ difference would have significantly impacted the findings. 
In the current study, rats were substantially younger and a different strain than rats used by Sanchez et al (2008). Sanchez et al (2008) used 14 week old male adult Wistar rats while those used in the current study were only 7-8 week old Sprague-Dawley rats. Age has been shown to have a significant impact on TPH activity and 5HT synthesis (Esteban et al, 2004). The response to changes in dietary tryptophan also differs between Wistar and Sprague-Dawley rats, suggesting that there may be differences in tryptophan absorption or metabolism between strains (Hayakawa et al, 1991) that could explain the discrepancy.

It is important to note that in the majority of studies employing tryptophan to produce substantial increases in tissue levels of 5HT used a lengthy 6 week daily treatment protocol (Haider et al, 2006; 2007; Esteban et al, 2004; 2010). In fact, the protocol selected for the current study (Sanches et al, 2008) was chosen because tryptophan loading took place over 7 days, which fell within the time period that MDMA produced 5HT deficits. Therefore, attempts at reversing deficits may have been successful if a lengthier dosing regimen had been used.

It has been suggested that tryptophan metabolism is impaired in former-MDMA users as indicted by the relatively elevated free-tryptophan levels following chronic tryptophan exposure (Curran \& Verheyden, 2003). These differences were correlated to cognitive impairment in former users. In addition, the prolactin response (a 5HT system-mediated effect) to tryptophan was blunted in MDMA users (Price et al, 1989). Altered absorption or metabolism, could potentially explain why the current treatment failed to alter 5HT levels. Nevertheless, the current treatment also failed to alter tissue levels of 5HT in controls. Therefore, more stringent regimens of tryptophan loading may be required in order to adequately test it as a treatment to reverse MDMA-produced 5HT deficits. 


\section{Chapter 8: General Discussion}

The aim of this thesis was two-fold; to determine both the behavioural and neurochemical consequences of self-administered MDMA in rats, and to assess the potential of pharmacological manipulations to facilitate recovery from MDMA-produced neurochemical damage. Over the last few decades, investigation into the long-term effects of MDMA has demonstrated persistent changes in the 5HT system in Ecstasy users. These alterations may underlie a spectrum of behavioural abnormalities, including cognitive deficits. Despite a well-documented and generally accepted theme of detrimental consequences in Ecstasy users, the findings from human studies do not quite match up to the severe and robust neurochemical damage produced in animal studies. This disparity has led to concerns about the use of animal models to investigate effects of MDMA exposure, with questions raised about the relevance of findings to users and abusers. Nevertheless, animal models of MDMA-produced deficits are necessary for preclinical investigation of neuroadaptations which may underpin alterations in the 5HT system.

Self-administered MDMA produced a deficit in performance in the NOR task. Under the present experimental conditions, the dependent measure of number of approaches to objects was used to produce a forgetting function, depicting the decay of working memory. According to this function, working memory for the familiar object was no longer intact following an ITI of 15 minutes. This is consistent with studies that measure working memory in other tasks, in which retention intervals range in the order of seconds and minutes. In the human literature, NOR testing does not exceed 30 minutes in infants and 1 hour in adults (Ennaceur, 2010).

Typically, NOR testing in infants follows ITIs of between 10 seconds to 10 minutes (Fagan, 1974; Diamond, 1995; MacKay-Soroka, 1982; Rose et al, 1983). Therefore, it is surprising that NOR testing in rats is conducted using ITIs as long as 48 hours in some studies (Reviewed in Antunes \& Biala, 2011). On the other hand, majority of studies in rats use exploration time as a measure of exploratory behaviour and this could explain the discrepancy. 
It is possible that the dependent measure used in the current study is more analogous to behavioural measures in infant NOR testing.

MDMA self-administration produced ITI-independent NOR deficits that recovered within 10 weeks following self-administration. These data suggest that impaired performance was due to alterations in cognitive processes other than memory. Impairments in NOR performance may be underpinned by disrupted perceptual, motivational, attentional, locomotor, sensory or memory processes (Clarke et al, 2005). There is evidence in the human literature suggesting impaired attentional processing in Ecstasy users (Indelkofer et al, 2009; McCardle et al, 2004; Zakzanis et al, 2002) and recently, studies have shown that MDMA produced attentional deficits in rats (Piper et al, 2005; Dalley et al, 2007). Possible attention, motivation or locomotor deficits were, however, accounted for by demonstrating similar overall exploratory behaviour towards objects in MDMA-treated rats and controls.

Deficits in visual encoding may not be so easily detected by simply measuring exploratory parameters like number of approaches or time spent with objects. In the present study, NOR deficits may be reflective of impairments in visual processing. Indeed, alterations in visual cortical excitability (Oliveri \& Calvo, 2003; Dafters et al, 1999), and impaired visual perceptual (Brown et al, 2007) and information coding (McCardle et al, 2004) processes have been observed in Ecstasy users.

Because the NOR task relies heavily on the natural propensity to explore novel objects (Ennaceur, 2010), it is also possible that NOR deficits may result from changes in novelty-seeking behaviour. Novelty-seeking behaviour is altered in Ecstasy users (Butler \& Montgomery, 2004; Gerra et al, 1998), but some suggest that this behavioural abnormality is a predictor for use, rather than a consequence of use (Wu et al, 2010; Martins et al, 2008). In the present study, however, behaviour towards novel objects in MDMA self-administering rats was similar to controls when tested 10 weeks after last exposure. This suggests that a modified response to novelty is not a pre-existing condition in MDMA self-administering rats. Therefore, MDMA exposure may alter novelty-seeking behaviour transiently, thereby impairing performance in the NOR task. 
Another interpretation of the present study is that ITI-dependent deficits were not observed due to the 'floor effect' at ITIs longer than 1 minute. This issue could be reconciled if NOR testing could be conducted using shorter intervals i.e 10s and 30s. The logistics of such testing is difficult as the interval may not be sufficient for experimental conditions to be set up during T2. The possibility of such testing, however, should be explored further to allow for more definitive conclusions about the nature of NOR deficits to be reached. Regardless, the present study suggests that akin to findings from human studies, self-administered MDMA produced cognitive deficits that recovered over time.

MDMA self-administration also produced dose-dependent decreases in tissue levels of 5HT that recovered over time. The neurochemical deficits produced by MDMA self-administration were analogous to human data where despite high cumulative usage of MDMA, deficits were substantially less pronounced when compared to animal studies using comparable, if not smaller dosing regimens. It was speculated that this may be due to a preconditioning effect of small doses which were initially self-administered during earlier sessions. It is possible that neuroprotection is afforded in a similar way in humans. Because of the similarities between the selfadministration data obtained in the present investigation and human literature, these findings further support the use of the self-administration model to examine the long-term effects of MDMA.

The parameters of neurochemical deficits following MDMA selfadministration were not consistent with behavioural deficits. Selfadministered MDMA impaired NOR in rats 7 days after exposure, and there was recovery 70 days after exposure (Schenk, Harper \& Do, 2011). On the other hand, deficits in tissue levels of 5HT persisted for up to 10 weeks following exposure. This may indicate that either alterations in tissue levels of 5HT do not contribute to the NOR deficits, or that that the 5HT system confers its effects on NOR indirectly through intermediate systems or substrates. Tissue levels of 5HT have been implicated in NOR performance (King et al, 2009; Jenkins et al, 2010), and therefore it is unlikely that MDMA-produced neurochemical and behavioural deficits are dissociable. 
Deficits in tissue levels of 5HT were present at the start of NOR testing (70 days following MDMA exposure). It is possible, however, that tissue levels of 5HT recovered between 70 and 77 days when NOR testing was conducted, and this underpinned the recovery of NOR performance. To test this, future directions could involve conducting neurochemical assays at 74 days or 77 days following MDMA self-administration.

One downside to MDMA administration compared to other drug exposure regimens is that it is time consuming as well as laborious. Combined with an acquisition rate of approximately $50 \%$, complications with catheter patency make the output for usable subjects relatively low compared to other methods of drug administration. Furthermore, on average, in the current study, 35-40 sessions of self-administration was required for subjects to achieve the high criterion. Depending on the available equipment for self-administration testing (i.e. self-administration chambers), this could entail a very lengthy process. Therefore, while this model of drug intake represents a very useful model for pre-clinical research, it is perhaps not the most ideal model of MDMA exposure for initial preliminary research. Instead, a standard procedure, in which large doses of MDMA are administered parenterally by the experimenter over a few days, may represent a more suitable animal model for the early stages of investigations.

It is noteworthy, however, that there are important differences between experimenter-administration and self-administration studies, not only pertaining to method of drug exposure. For example, factors such as stress and daily handling can influence the outcome of experiments. Importantly, the rats used for the experimenter-administered MDMA studies were also substantially younger than those used in self-administration experiments. It is necessary to use young rats for experimenteradministration experiments, as the mortality rate increases in older rats. This difference in age, however, could impact the effect of different treatments being tested or pharmacological manipulation. Therefore, it might be useful to develop a protocol for MDMA administration that would be more comparable to self-administration studies. For instance, MDMA could be administered daily over a period of time, at increasing dose, to reflect patterns of drug exposure observed in self-administration experiments. 
Nevertheless, the self-administration model of MDMA exposure represents a necessary step between these preliminary studies and clinical research. The current investigation shows that the self-administration data may be more akin to the human literature where despite the lack of pronounced neurochemical damage, functional consequences appear to persist possibly due to important yet enduring subtle changes in the brain (Reviewed in Gouzoulis-Mayfrank et al, 2006).

A second major theme to this thesis was exploration into the development of treatments to reverse MDMA-produced 5HT deficits, on which there has been little research. Numerous studies have investigated the efficacy of protective compounds against damage, but this approach is problematic. Prior or concomitant treatment of compounds with MDMA may change the pharmacokinetics and pharmacodynamics of MDMA, potentially making it more dangerous (Upreti \& Edington, 2008; Hewton et al, 2007; Stanley et al, 2007; Freezer et al, 2005; Vuori et al, 2003; Copeland et al, 2006) or blunting the positive subjective effects (Tancer \& Johnsson, 2007; Farre et al, 2007; Liechti et al, 2000), thus making it undesirable. Therefore, it would be valuable to elucidate the underlying mechanisms of MDMA-produced 5HT deficits in order to target them. This could lead to the development of pharmacological therapy for MDMA users who experience problems.

The current experiments were designed to further explore the potential candidacy of three different compounds to reverse the MDMAproduced decrease in tissue levels of 5HT. Because of the well-established role of the $5 \mathrm{HT}_{1 \mathrm{a}}$ autoreceptor in regulating $5 \mathrm{HT}$ synthesis, it was hypothesised that chronic MDMA exposure supersensitises the autoreceptor, thus decreasing tissue levels of 5HT. MDMA exposure, either by experimenter-administration or self-administration, did not alter the sensitivity of the $5 \mathrm{HT}_{1 \mathrm{a}}$ autoreceptor, as measured by the LLR response to low doses of 8-OH-DPAT. A 5 $\mathrm{HT}_{1 \mathrm{a}}$ autoreceptor-desensitizing regimen of 8-OH-DPAT did not reverse or attenuate the effects of MDMA selfadministration on tissue levels of 5HT in the brain, further negating a role for the $5 \mathrm{HT}_{1 \mathrm{a}}$ autoreceptor. This treatment produced a rightwards shift in the dose response curve, suggesting a desensitisation. Taken together, these 
findings are consistent with the idea that MDMA exposure does not supersensitise the $5 \mathrm{HT}_{1 \mathrm{a}}$ autoreceptor, and this is not a viable pharmacological target for reversing or attenuating MDMA-produced deficits in tissue $5 \mathrm{HT}$.

This was compounded by the inability of chronic trazodone treatment, which had previously been reported to desensitize the $5 \mathrm{HT}_{1 \mathrm{a}}$ autoreceptor, to reverse the MDMA-produced decrease in tissue 5HT levels. Because MDMA had also been shown to attenuate the effects of fluoxetine in previous studies, it is possible that the MDMA exposure blunted the effect of trazodone. For example, chronic trazodone altered 5HT turnover in the current study, and was therefore pharmacologically effective. On the other hand, it is possible that the treatment did not desensitise the $5 \mathrm{HT}_{1 \mathrm{a}}$ autoreceptor in MDMA-treated rats. Perhaps more stringent dosing regimens are required in subjects previously exposed to MDMA to enhance serotonergic transmission. Anti-depressants function through a wide range of pharmacological mechanisms to restore serotonergic function in depressed patients and it is possible that some may be more efficacious than others in treating MDMA-produced damage. To date, however, there is very little preclinical investigation into this possibility and future directions could entail exploring the efficacy of different anti-depressant treatments in returning tissue levels of 5HT to normal, following MDMA exposure.

Serotonin synthesis is determined by the availability of the precursor, tryptophan (Choi et al, 2011; Curzon G., 1981; Young \& Gauthier, 1981; Moja et al, 1989; Gartside et al, 1992; Estaban et al, 2004). A tryptophanfree diet decreased tissue levels of 5HT (Boulin DJ, 1963) and chronic treatment with L-tryptophan increased tissue levels of 5HT (Sanchez et al, 2008; Coskun et al, 2006; Khaliq et al, 2007; Haider et al, 2006; 2007; Hansen et al, 2011; Estaban et al, 2010). Therefore, tissue levels of 5HT following MDMA exposure, should also be sensitive to administration of tryptophan. In humans, tryptophan loading alters mood, cognition and sleep and the sensitivity to this effect is dependent on the initial state of the 5HT system; tryptophan loading was more effective in improving sleep, mood and cognition in vulnerable subjects (Silber \& Schmitt, 2010). Tryptophan loading has also been investigated as a clinical treatment for psychological 
dysfunctions including depression (Meyers, 2005) and schizophrenia (Bigelow et al, 1979), due to the involvement of the 5HT system in these diseases. Furthermore, tryptophan restored tissue levels of 5HT and TPH activity to normal following chronic methamphetamine administration (Trulson \& Trulson, 1982).

Under the present experiment conditions, tryptophan loading did not affect tissue levels of 5HT in MDMA-treated or drug-naïve rats. One limitation of the current study was the short window of time in which the treatment could be administered ( 2 weeks), prior to neurochemical assays. Majority of protocols in the literature suggest extensive treatment (6 weeks) to alter tissue levels of 5HT, but we could only use a 7 day protocol of oral tryptophan administration. The use of an oral administration protocol was appealing as this most reflected a simple form of administration in a clinical setting, which was the ultimate aim of this investigation. On the other hand, tryptophan absorption following oral administration may be impaired in Ecstasy users. Therefore, administration via another route may be more appropriate for treating MDMA-produced 5HT deficits Ecstasy users. It is also possible that the dosing regimen used was too low to produce any changes in tissue 5HT levels following MDMA exposure, since it failed to alter tissue 5HT levels in drug-naïve controls. It is interesting to note that the effects of tryptophan on tissue levels of 5HT is sensitive to time of administration, age and possibly strain.

In order to develop a dosing regimen that would be effective in increasing tissue levels of 5HT in both drug-naive and MDMA-exposed rats, various parameters could be manipulated. For example, as previously suggested, it is possible that the oral route of administration may not be suitable for tryptophan to be effective in MDMA-treated rats. Parental routes of administration such as intraperitoneal or subcutaneous could be tested and intravenous injections would increase bioavailability of tryptophan substantially. Acutely, intravenous L-tryptophan produced a marked increase in tissue levels of 5HT (Molina et al, 2001), and therefore chronic intravenous treatment may produce a long-term effect in MDMAtreated rats. This route of administration would be particularly appealing in 
experiments using MDMA self-administration due to the presence of the indwelling catheter.

A more stringent dosing regimen may be able to increase tissue levels of 5HT in both MDMA-treated and drug-naive rats. This can be achieved by either increasing the daily dose of tryptophan or the duration of the protocol. The duration of the dosing regimen, however, is limited by the window of time between MDMA exposure and recovery of tissue levels of $5 \mathrm{HT}$. Therefore, a better option for testing under the present experimental conditions may be to increase the daily dose of tryptophan administered. These possibilities all entail future directions of the current research and may eventually lead to a viable pharmacological treatment to provide therapeutic aid for MDMA users.

\section{Conclusions}

The present study suggests that MDMA self-administration in rats produces neurochemical deficits that are subtle, and yet may translate into pronounced behavioural abnormalities. Therefore, it is imperative to understand how MDMA confers these effects if clinical treatments are to be developed. While the role of the $5 \mathrm{HT}_{1 \mathrm{a}}$ autoreceptor in mediating these effects was not supported by the current findings, the use of precursors and antidepressant treatments still present themselves as highly useful possibilities for these treatments and should be explored further. The MDMA self-administration model in rats is a useful preclinical model for this investigation. 


\section{References}

Abdallah, A.B., Scheier, L.M., Inciardi, J.A., Copeland, J. \& Cottler, L.B. (2007) A psycho-economic model of ecstasy consumption and related consequences: a multi-site study with community samples. Substance use \& misuse, 42, 1651-1684.

Able, J.A., Gudelsky, G.A., Vorhees, C.V. \& Williams, M.T. (2006) 3,4Methylenedioxymethamphetamine in adult rats produces deficits in path integration and spatial reference memory. Biological psychiatry, 59, 1219-1226.

Adamaszek, M., Khaw, A.V., Buck, U., Andresen, B. \& Thomasius, R. (2010) Evidence of neurotoxicity of ecstasy: sustained effects on electroencephalographic activity in polydrug users. PloS one, 5, e14097.

Adori, C., Ando, R.D., Ferrington, L., Szekeres, M., Vas, S., Kelly, P.A., Hunyady, L. \& Bagdy, G. Elevated BDNF protein level in cortex but not in hippocampus of MDMA-treated Dark Agouti rats: a potential link to the long-term recovery of serotonergic axons. Neuroscience letters, 478, 56-60.

Adori, C., Ando, R.D., Ferrington, L., Szekeres, M., Vas, S., Kelly, P.A., Hunyady, L. \& Bagdy, G. (2010) Elevated BDNF protein level in cortex but not in hippocampus of MDMA-treated Dark Agouti rats: a potential link to the long-term recovery of serotonergic axons. Neuroscience letters, 478, 56-60.

Adori, C., Ando, R.D., Kovacs, G.G. \& Bagdy, G. (2006) Damage of serotonergic axons and immunolocalization of Hsp27, Hsp72, and Hsp90 molecular chaperones after a single dose of MDMA administration in Dark Agouti rat: temporal, spatial, and cellular patterns. The Journal of comparative neurology, 497, 251-269.

Adori, C., Ando, R.D., Szekeres, M., Gutknecht, L., Kovacs, G.G., Hunyady, L., Lesch, K.P. \& Bagdy, G. Recovery and aging of serotonergic fibers after single and intermittent MDMA treatment in Dark Agouti rat. The Journal of comparative neurology, 519, 2353 2378 .

Adori, C., Ando, R.D., Szekeres, M., Gutknecht, L., Kovacs, G.G., Hunyady, L., Lesch, K.P. \& Bagdy, G. (2011) Recovery and aging of serotonergic fibers after single and intermittent MDMA treatment in Dark Agouti rat. The Journal of comparative neurology, 519, 2353-2378.

Aguirre, N., Ballaz, S., Lasheras, B. \& Del Rio, J. (1998) MDMA ('Ecstasy') enhances 5-HT1A receptor density and 8-OH-DPAT-induced 
hypothermia: blockade by drugs preventing 5-hydroxytryptamine depletion. European journal of pharmacology, 346, 181-188.

Aguirre, N., Galbete, J.L., Lasheras, B. \& Del Rio, J. (1995) Methylenedioxymethamphetamine induces opposite changes in central pre- and postsynaptic 5-HT1A receptors in rats. European journal of pharmacology, 281, 101-105.

Aguirre, N., Galbete, J.L., Lasheras, B. \& Del Rio, J. (1995) Methylenedioxymethamphetamine induces opposite changes in central pre- and postsynaptic 5-HT1A receptors in rats. European journal of pharmacology, 281, 101-105.

Ainge, J.A., Heron-Maxwell, C., Theofilas, P., Wright, P., de Hoz, L. \& Wood, E.R. (2006) The role of the hippocampus in object recognition in rats: examination of the influence of task parameters and lesion size. Behavioural brain research, 167, 183-195.

Albasser, M.M., Davies, M., Futter, J.E. \& Aggleton, J.P. (2009) Magnitude of the object recognition deficit associated with perirhinal cortex damage in rats: Effects of varying the lesion extent and the duration of the sample period. Behavioral neuroscience, 123, 115-124.

Allen, R.P., McCann, U.D. \& Ricaurte, G.A. (1993) Persistent effects of (+/- )3,4-methylenedioxymethamphetamine (MDMA, "ecstasy") on human sleep. Sleep, 16, 560-564.

Ando, R.D., Benko, A., Ferrington, L., Kirilly, E., Kelly, P.A. \& Bagdy, G. (2006) Partial lesion of the serotonergic system by a single dose of MDMA results in behavioural disinhibition and enhances acute MDMA-induced social behaviour on the social interaction test. Neuropharmacology, 50, 884-896.

Antunes, M. \& Biala, G. (2011) The novel object recognition memory: neurobiology, test procedure, and its modifications. Cognitive processing.

Arias-Cavieres, A., Rozas, C., Reyes-Parada, M., Barrera, N., Pancetti, F., Loyola, S., Lorca, R.A., Zeise, M.L. \& Morales, B. (2010) MDMA ("ecstasy") impairs learning in the Morris Water Maze and reduces hippocampal LTP in young rats. Neuroscience letters, 469, 375-379.

Ator, N.A. \& Griffiths, R.R. (1983) Nicotine self-administration in baboons. Pharmacology, biochemistry, and behavior, 19, 993-1003.

Aura, J., Sirvio, J. \& Riekkinen, P., Jr. (1997) Methoctramine moderately improves memory but pirenzepine disrupts performance in delayed non-matching to position test. European journal of pharmacology, 333, 129-134. 
Back-Madruga, C., Boone, K.B., Chang, L., Grob, C.S., Lee, A., Nations, H. \& Poland, R.E. (2003) Neuropsychological effects of 3,4methylenedioxymethamphetamine (MDMA or ecstasy) in recreational users. The Clinical neuropsychologist, 17, 446-459.

Bahora, M., Sterk, C.E. \& Elifson, K.W. (2009) Understanding recreational ecstasy use in the United States: a qualitative inquiry. The International journal on drug policy, 20, 62-69.

Bahrick, L.E. \& Pickens, J.N. (1995) Infant memory for object motion across a period of three months: implications for a four-phase attention function. Journal of experimental child psychology, 59, 343-371.

Balster, R.L., Kilbey, M.M. \& Ellinwood, E.H., Jr. (1976) Methamphetamine self-administration in the cat. Psychopharmacologia, 46, 229-233.

Balster, R.L., Kilbey, M.M. \& Ellinwood, E.H., Jr. (1976) Methamphetamine self-administration in the cat. Psychopharmacologia, 46, 229-233.

Banks, M.L., Czoty, P.W., Gage, H.D., Bounds, M.C., Garg, P.K., Garg, S. \& Nader, M.A. (2008) Effects of cocaine and MDMA selfadministration on serotonin transporter availability in monkeys. Neuropsychopharmacology, 33, 219-225.

Banks, M.L., Czoty, P.W., Gage, H.D., Bounds, M.C., Garg, P.K., Garg, S. \& Nader, M.A. (2008) Effects of cocaine and MDMA selfadministration on serotonin transporter availability in monkeys. Neuropsychopharmacology, 33, 219-225.

Battaglia, G., Brooks, B.P., Kulsakdinun, C. \& De Souza, E.B. (1988) Pharmacologic profile of MDMA (3,4methylenedioxymethamphetamine) at various brain recognition sites. European journal of pharmacology, 149, 159-163.

Battaglia, G., Yeh, S.Y. \& De Souza, E.B. (1988) MDMA-induced neurotoxicity: parameters of degeneration and recovery of brain serotonin neurons. Pharmacology, biochemistry, and behavior, 29, 269-274.

Battaglia, G., Yeh, S.Y. \& De Souza, E.B. (1988) MDMA-induced neurotoxicity: parameters of degeneration and recovery of brain serotonin neurons. Pharmacology, biochemistry, and behavior, 29, 269-274.

Bauernfeind, A.L., Dietrich, M.S., Blackford, J.U., Charboneau, E.J., Lillevig, J.G., Cannistraci, C.J., Woodward, N.D., Cao, A., Watkins, T., Di Iorio, C.R., Cascio, C., Salomon, R.M. \& Cowan, R.L. (2011) 
Human ecstasy use is associated with increased cortical excitability: an fMRI study. Neuropsychopharmacology, 36, 1127-1141.

Baumann, M.H., Clark, R.D., Franken, F.H., Rutter, J.J. \& Rothman, R.B. (2008) Tolerance to 3,4-methylenedioxymethamphetamine in rats exposed to single high-dose binges. Neuroscience, 152, 773-784.

Baumann, M.H., Clark, R.D. \& Rothman, R.B. (2008) Locomotor stimulation produced by 3,4-methylenedioxymethamphetamine (MDMA) is correlated with dialysate levels of serotonin and dopamine in rat brain. Pharmacology, biochemistry, and behavior, 90, 208-217.

Baumann, M.H., Wang, X. \& Rothman, R.B. (2007) 3,4Methylenedioxymethamphetamine (MDMA) neurotoxicity in rats: a reappraisal of past and present findings. Psychopharmacology, 189, 407-424.

Baumann, M.H., Zolkowska, D., Kim, I., Scheidweiler, K.B., Rothman, R.B. \& Huestis, M.A. (2009) Effects of dose and route of administration on pharmacokinetics of $(+$ or -$)-3,4-$ methylenedioxymethamphetamine in the rat. Drug metabolism and disposition: the biological fate of chemicals, 37, 2163-2170.

Beardsley, P.M., Balster, R.L. \& Harris, L.S. (1986) Self-administration of methylenedioxymethamphetamine (MDMA) by rhesus monkeys. Drug and alcohol dependence, 18, 149-157.

Beck, K.D. \& Luine, V.N. (1999) Food deprivation modulates chronic stress effects on object recognition in male rats: role of monoamines and amino acids. Brain research, 830, 56-71.

Belcher, A.M., Feinstein, E.M., O'Dell, S.J. \& Marshall, J.F. (2008) Methamphetamine influences on recognition memory: comparison of escalating and single-day dosing regimens. Neuropsychopharmacology, 33, 1453-1463.

Belcher, A.M., O'Dell, S.J. \& Marshall, J.F. (2005) Impaired object recognition memory following methamphetamine, but not $\mathrm{p}$ chloroamphetamine- or d-amphetamine-induced neurotoxicity. Neuropsychopharmacology, 30, 2026-2034.

Benzenhofer, U. \& Passie, T. (2010) Rediscovering MDMA (ecstasy): the role of the American chemist Alexander T. Shulgin. Addiction, 105, 1355-1361.

Berendsen, H.H., Bourgondien, F.G. \& Broekkamp, C.L. (1994) Role of dorsal and median raphe nuclei in lower lip retraction in rats. European journal of pharmacology, 263, 315-318. 
Berendsen, H.H. \& Broekkamp, C.L. (1991) Attenuation of 5-HT1A and 5HT2 but not 5-HT1C receptor mediated behaviour in rats following chronic treatment with 5-HT receptor agonists, antagonists or antidepressants. Psychopharmacology, 105, 219-224.

Berendsen, H.H., Broekkamp, C.L. \& van Delft, A.M. (1991) Depletion of brain serotonin differently affects behaviors induced by 5HT1A, 5HT1C, and 5HT2 receptor activation in rats. Behavioral and neural biology, 55, 214-226.

Berger, U.V., Gu, X.F. \& Azmitia, E.C. (1992) The substituted amphetamines 3,4-methylenedioxymethamphetamine, methamphetamine, p-chloroamphetamine and fenfluramine induce 5-hydroxytryptamine release via a common mechanism blocked by fluoxetine and cocaine. European journal of pharmacology, 215, 153-160.

Bertaina-Anglade, V., Enjuanes, E., Morillon, D. \& Drieu la Rochelle, C. (2006) The object recognition task in rats and mice: a simple and rapid model in safety pharmacology to detect amnesic properties of a new chemical entity. Journal of pharmacological and toxicological methods, 54, 99-105.

Bevins, R.A. \& Besheer, J. (2006) Object recognition in rats and mice: a one-trial non-matching-to-sample learning task to study 'recognition memory'. Nature protocols, 1, 1306-1311.

Bhattachary, S. \& Powell, J.H. (2001) Recreational use of 3,4methylenedioxymethamphetamine (MDMA) or 'ecstasy': evidence for cognitive impairment. Psychological medicine, 31, 647-658.

Bhide, N.S., Lipton, J.W., Cunningham, J.I., Yamamoto, B.K. \& Gudelsky, G.A. (2009) Repeated exposure to MDMA provides neuroprotection against subsequent MDMA-induced serotonin depletion in brain. Brain research, 1286, 32-41.

Biezonski, D.K. \& Meyer, J.S. Effects of 3,4methylenedioxymethamphetamine (MDMA) on serotonin transporter and vesicular monoamine transporter 2 protein and gene expression in rats: implications for MDMA neurotoxicity. Journal of neurochemistry, 112, 951-962.

Biezonski, D.K. \& Meyer, J.S. (2010) Effects of 3,4methylenedioxymethamphetamine (MDMA) on serotonin transporter and vesicular monoamine transporter 2 protein and gene expression in rats: implications for MDMA neurotoxicity. Journal of neurochemistry, 112, 951-962. 
Bigelow, L.B., Walls, P., Gillin, J.C. \& Wyatt, R.J. (1979) Clinical effects of L-5-hydroxytryptophan administration in chronic schizophrenic patients. Biological psychiatry, 14, 53-67.

Bisagno, V., Ferguson, D. \& Luine, V.N. (2002) Short toxic methamphetamine schedule impairs object recognition task in male rats. Brain research, 940, 95-101.

Blagrove, M., Seddon, J., George, S., Parrott, A.C., Stickgold, R., Walker, M.P., Jones, K.A. \& Morgan, M.J. (2011) Procedural and declarative memory task performance, and the memory consolidation function of sleep, in recent and abstinent ecstasy/MDMA users. J Psychopharmacol, 25, 465-477.

Blier, P. (2010) Altered function of the serotonin 1A autoreceptor and the antidepressant response. Neuron, 65, 1-2.

Blier, P., Lista, A. \& De Montigny, C. (1993) Differential properties of preand postsynaptic 5-hydroxytryptamine1A receptors in the dorsal raphe and hippocampus: II. Effect of pertussis and cholera toxins. The Journal of pharmacology and experimental therapeutics, 265, 16-23.

Blokhina, E.A., Dravolina, O.A., Bespalov, A.Y., Balster, R.L. \& Zvartau, E.E. (2004) Intravenous self-administration of abused solvents and anesthetics in mice. European journal of pharmacology, 485, 211218.

Boadle-Biber, M.C. (1993) Regulation of serotonin synthesis. Progress in biophysics and molecular biology, 60, 1-15.

Boadle-Biber, M.C., Singh, V.B., Corley, K.C., Phan, T.H. \& Dilts, R.P. (1993) Evidence that corticotropin-releasing factor within the extended amygdala mediates the activation of tryptophan hydroxylase produced by sound stress in the rat. Brain research, $\mathbf{6 2 8}$, 105-114.

Bogen, I.L., Haug, K.H., Myhre, O. \& Fonnum, F. (2003) Short- and longterm effects of MDMA ("ecstasy") on synaptosomal and vesicular uptake of neurotransmitters in vitro and ex vivo. Neurochemistry international, 43, 393-400.

Bohmaker, K., Eison, A.S., Yocca, F.D. \& Meller, E. (1993) Comparative effects of chronic 8-OH-DPAT, gepirone and ipsapirone treatment on the sensitivity of somatodendritic 5-HT1A autoreceptors. Neuropharmacology, 32, 527-534.

Bolla, K.I., McCann, U.D. \& Ricaurte, G.A. (1998) Memory impairment in abstinent MDMA ("Ecstasy") users. Neurology, 51, 1532-1537. 
Bonkale, W.L. \& Austin, M.C. (2008) 3,4Methylenedioxymethamphetamine induces differential regulation of tryptophan hydroxylase 2 protein and mRNA levels in the rat dorsal raphe nucleus. Neuroscience, 155, 270-276.

Bonkale, W.L. \& Austin, M.C. (2008) 3,4Methylenedioxymethamphetamine induces differential regulation of tryptophan hydroxylase 2 protein and mRNA levels in the rat dorsal raphe nucleus. Neuroscience, 155, 270-276.

Briand, L.A., Gross, J.P. \& Robinson, T.E. (2008) Impaired object recognition following prolonged withdrawal from extended-access cocaine self-administration. Neuroscience, 155, 1-6.

Brown, J., McKone, E. \& Ward, J. (2010) Deficits of long-term memory in ecstasy users are related to cognitive complexity of the task. Psychopharmacology, 209, 51-67.

Buchert, R., Thomasius, R., Nebeling, B., Petersen, K., Obrocki, J., Jenicke, L., Wilke, F., Wartberg, L., Zapletalova, P. \& Clausen, M. (2003) Long-term effects of "ecstasy" use on serotonin transporters of the brain investigated by PET. Journal of nuclear medicine : official publication, Society of Nuclear Medicine, 44, 375-384.

Buchert, R., Thomasius, R., Petersen, K., Wilke, F., Obrocki, J., Nebeling, B., Wartberg, L., Zapletalova, P. \& Clausen, M. (2006) Reversibility of ecstasy-induced reduction in serotonin transporter availability in polydrug ecstasy users. European journal of nuclear medicine and molecular imaging, 33, 188-199.

Buchert, R., Thomasius, R., Wilke, F., Petersen, K., Nebeling, B., Obrocki, J., Schulze, O., Schmidt, U. \& Clausen, M. (2004) A voxel-based PET investigation of the long-term effects of "Ecstasy" consumption on brain serotonin transporters. The American journal of psychiatry, 161, 1181-1189.

Butler, G.K. \& Montgomery, A.M. (2004) Impulsivity, risk taking and recreational 'ecstasy' (MDMA) use. Drug and alcohol dependence, 76, 55-62.

Callahan, B.T., Cord, B.J. \& Ricaurte, G.A. (2001) Long-term impairment of anterograde axonal transport along fiber projections originating in the rostral raphe nuclei after treatment with fenfluramine or methylenedioxymethamphetamine. Synapse (New York, N.Y, 40, 113-121.

Callahan, B.T., Cord, B.J. \& Ricaurte, G.A. (2001) Long-term impairment of anterograde axonal transport along fiber projections originating in the rostral raphe nuclei after treatment with fenfluramine or 
methylenedioxymethamphetamine. Synapse (New York, N.Y, 40, 113-121.

Camarasa, J., Marimon, J.M., Rodrigo, T., Escubedo, E. \& Pubill, D. (2008) Memantine prevents the cognitive impairment induced by 3,4methylenedioxymethamphetamine in rats. European journal of pharmacology, 589, 132-139.

Carhart-Harris, R.L., Nutt, D.J., Munafo, M. \& Wilson, S.J. (2009) Current and former ecstasy users report different sleep to matched controls: a web-based questionnaire study. J Psychopharmacol, 23, 249-257.

Carney, J.M., Landrum, R.W., Cheng, M.S. \& Seale, T.W. (1991) Establishment of chronic intravenous drug self-administration in the C57BL/6J mouse. Neuroreport, 2, 477-480.

Carnicella, S., Yowell, Q.V. \& Ron, D. (2011) Regulation of operant oral ethanol self-administration: a dose-response curve study in rats. Alcoholism, clinical and experimental research, 35, 116-125.

Cassel, J.C., Riegert, C., Rutz, S., Koenig, J., Rothmaier, K., Cosquer, B., Lazarus, C., Birthelmer, A., Jeltsch, H., Jones, B.C. \& Jackisch, R. (2005) Ethanol, 3,4-methylenedioxymethamphetamine (ecstasy) and their combination: long-term behavioral, neurochemical and neuropharmacological effects in the rat. Neuropsychopharmacology, 30, 1870-1882.

Cha, X.Y., Pierce, R.C., Kalivas, P.W. \& Mackler, S.A. (1997) NAC-1, a rat brain mRNA, is increased in the nucleus accumbens three weeks after chronic cocaine self-administration. J Neurosci, 17, 6864-6871.

Chamberlain, J., Offord, S.J., Wolfe, B.B., Tyau, L.S., Wang, H.L. \& Frazer, A. (1993) Potency of 5-hydroxytryptamine1a agonists to inhibit adenylyl cyclase activity is a function of affinity for the "lowaffinity" state of $[3 \mathrm{H}] 8$-hydroxy-N,N-dipropylaminotetralin $([3 \mathrm{H}] 8$ OH-DPAT) binding. The Journal of pharmacology and experimental therapeutics, 266, 618-625.

Chami, G., Jeys, L., Freudmann, M., Connor, L. \& Siddiqi, M. (2006) Are osteoporotic fractures being adequately investigated? A questionnaire of GP \& orthopaedic surgeons. BMC family practice, 7, 7 .

Chang, L., Ernst, T., Grob, C.S. \& Poland, R.E. (1999) Cerebral (1)H MRS alterations in recreational 3, 4-methylenedioxymethamphetamine (MDMA, "ecstasy") users. Journal of magnetic resonance imaging : JMRI, 10, 521-526.

Chang, L., Grob, C.S., Ernst, T., Itti, L., Mishkin, F.S., Jose-Melchor, R. \& Poland, R.E. (2000) Effect of ecstasy [3,4- 
methylenedioxymethamphetamine (MDMA)] on cerebral blood flow: a co-registered SPECT and MRI study. Psychiatry research, 98, 1528.

Chaput, Y., de Montigny, C. \& Blier, P. (1986) Effects of a selective 5-HT reuptake blocker, citalopram, on the sensitivity of 5-HT autoreceptors: electrophysiological studies in the rat brain. NaunynSchmiedeberg's archives of pharmacology, 333, 342-348.

Choi, S., DiSilvio, B., Fernstrom, M.H. \& Fernstrom, J.D. (2011) The chronic ingestion of diets containing different proteins produces marked variations in brain tryptophan levels and serotonin synthesis in the rat. Neurochemical research, 36, 559-565.

Chu, T., Kumagai, Y., DiStefano, E.W. \& Cho, A.K. (1996) Disposition of methylenedioxymethamphetamine and three metabolites in the brains of different rat strains and their possible roles in acute serotonin depletion. Biochemical pharmacology, 51, 789-796.

Chudasama, Y. \& Muir, J.L. (1997) A behavioural analysis of the delayed non-matching to position task: the effects of scopolamine, lesions of the fornix and of the prelimbic region on mediating behaviours by rats. Psychopharmacology, 134, 73-82.

Chummun, H., Tilley, V. \& Ibe, J. (2010) 3,4-methylenedioxyamfetamine (ecstasy) use reduces cognition. Br J Nurs, 19, 94-100.

Chummun, H., Tilley, V. \& Ibe, J. (2010) 3,4-methylenedioxyamfetamine (ecstasy) use reduces cognition. Br J Nurs, 19, 94-100.

Clark, R.E., Kuczenski, R. \& Segal, D.S. (2007) Escalating dose, multiple binge methamphetamine regimen does not impair recognition memory in rats. Synapse (New York, N.Y, 61, 515-522.

Clarke, W.P., Yocca, F.D. \& Maayani, S. (1996) Lack of 5hydroxytryptamine1A-mediated inhibition of adenylyl cyclase in dorsal raphe of male and female rats. The Journal of pharmacology and experimental therapeutics, 277, 1259-1266.

Clemens, K.J., Cornish, J.L., Hunt, G.E. \& McGregor, I.S. (2007) Repeated weekly exposure to MDMA, methamphetamine or their combination: long-term behavioural and neurochemical effects in rats. Drug and alcohol dependence, 86, 183-190.

Codony, X., Vela, J.M. \& Ramirez, M.J. (2011) 5-HT(6) receptor and cognition. Current opinion in pharmacology, 11, 94-100.

Cohen, R.S. (1995) Subjective reports on the effects of the MDMA ('ecstasy') experience in humans. Progress in neuropsychopharmacology \& biological psychiatry, 19, 1137-1145. 
Colado, M.I., Esteban, B., O'Shea, E., Granados, R. \& Green, A.R. (1999) Studies on the neuroprotective effect of pentobarbitone on MDMAinduced neurodegeneration. Psychopharmacology, 142, 421-425.

Colado, M.I., Murray, T.K. \& Green, A.R. (1993) 5-HT loss in rat brain following 3,4-methylenedioxymethamphetamine (MDMA), pchloroamphetamine and fenfluramine administration and effects of chlormethiazole and dizocilpine. British journal of pharmacology, 108, 583-589.

Colado, M.I., Williams, J.L. \& Green, A.R. (1995) The hyperthermic and neurotoxic effects of 'Ecstasy' (MDMA) and 3,4 methylenedioxyamphetamine (MDA) in the Dark Agouti (DA) rat, a model of the CYP2D6 poor metabolizer phenotype. British journal of pharmacology, 115, 1281-1289.

Cole, J.C. \& Sumnall, H.R. (2003) The pre-clinical behavioural pharmacology of 3,4-methylenedioxymethamphetamine (MDMA). Neuroscience and biobehavioral reviews, 27, 199-217.

Commins, D.L., Vosmer, G., Virus, R.M., Woolverton, W.L., Schuster, C.R. \& Seiden, L.S. (1987) Biochemical and histological evidence that methylenedioxymethylamphetamine (MDMA) is toxic to neurons in the rat brain. The Journal of pharmacology and experimental therapeutics, 241, 338-345.

Copeland, J., Dillon, P. \& Gascoigne, M. (2006) Ecstasy and the concomitant use of pharmaceuticals. Addictive behaviors, 31, 367370 .

Corley, K.C., Singh, V.B., Phan, T.H. \& Boadle-Biber, M.C. (1992) Effect of gepirone on increases in tryptophan hydroxylase in response to sound stress. European journal of pharmacology, 213, 417-425.

Cornish, J.L., Shahnawaz, Z., Thompson, M.R., Wong, S., Morley, K.C., Hunt, G.E. \& McGregor, I.S. (2003) Heat increases 3,4methylenedioxymethamphetamine self-administration and social effects in rats. European journal of pharmacology, 482, 339-341.

Coskun, S., Ozer, C., Gonul, B., Take, G. \& Erdogan, D. (2006) The effect of repeated tryptophan administration on body weight, food intake, brain lipid peroxidation and serotonin immunoreactivity in mice. Molecular and cellular biochemistry, 286, 133-138.

Cottler, L.B., Leung, K.S. \& Abdallah, A.B. (2009) Test-re-test reliability of DSM-IV adopted criteria for 3,4methylenedioxymethamphetamine (MDMA) abuse and dependence: a cross-national study. Addiction, 104, 1679-1690. 
Cottler, L.B., Womack, S.B., Compton, W.M. \& Ben-Abdallah, A. (2001) Ecstasy abuse and dependence among adolescents and young adults: applicability and reliability of DSM-IV criteria. Human psychopharmacology, 16, 599-606.

Cowan, R.L., Lyoo, I.K., Sung, S.M., Ahn, K.H., Kim, M.J., Hwang, J., Haga, E., Vimal, R.L., Lukas, S.E. \& Renshaw, P.F. (2003) Reduced cortical gray matter density in human MDMA (Ecstasy) users: a voxel-based morphometry study. Drug and alcohol dependence, $\mathbf{7 2}$, 225-235.

Cowan, R.L., Lyoo, I.K., Sung, S.M., Ahn, K.H., Kim, M.J., Hwang, J., Haga, E., Vimal, R.L., Lukas, S.E. \& Renshaw, P.F. (2003) Reduced cortical gray matter density in human MDMA (Ecstasy) users: a voxel-based morphometry study. Drug and alcohol dependence, 72, 225-235.

Crespo, J.A., Manzanares, J., Oliva, J.M., Corchero, J., Garcia-Lecumberri, C. \& Ambrosio, E. (2003) Extinction of cocaine self-administration produces alterations in corticotropin releasing factor gene expression in the paraventricular nucleus of the hypothalamus. Brain research. Molecular brain research, 117, 160-167.

Crespo, J.A., Manzanares, J., Oliva, J.M., Corchero, J., Palomo, T. \& Ambrosio, E. (2001) Extinction of cocaine self-administration produces a differential time-related regulation of proenkephalin gene expression in rat brain. Neuropsychopharmacology, 25, 185-194.

Crespo, J.A., Oliva, J.M., Ghasemzadeh, M.B., Kalivas, P.W. \& Ambrosio, E. (2002) Neuroadaptive changes in NMDAR1 gene expression after extinction of cocaine self-administration. Annals of the New York Academy of Sciences, 965, 78-91.

Croft, R.J., Klugman, A., Baldeweg, T. \& Gruzelier, J.H. (2001) Electrophysiological evidence of serotonergic impairment in longterm MDMA ("ecstasy") users. The American journal of psychiatry, 158, 1687-1692.

Cunningham, K.A., Paris, J.M. \& Goeders, N.E. (1992) Chronic cocaine enhances serotonin autoregulation and serotonin uptake binding. Synapse (New York, N.Y, 11, 112-123.

Cunningham, K.A., Paris, J.M. \& Goeders, N.E. (1992) Chronic cocaine enhances serotonin autoregulation and serotonin uptake binding. Synapse (New York, N.Y, 11, 112-123.

Curran, H.V. \& Travill, R.A. (1997) Mood and cognitive effects of +/-3,4methylenedioxymethamphetamine (MDMA, 'ecstasy'): week-end 'high' followed by mid-week low. Addiction, 92, 821-831. 
Curran, H.V. \& Verheyden, S.L. (2003) Altered response to tryptophan supplementation after long-term abstention from MDMA (ecstasy) is highly correlated with human memory function. Psychopharmacology, 169, 91-103.

Curzon, G. (1981) Influence of plasma tryptophan on brain 5HT synthesis and serotonergic activity. Advances in experimental medicine and biology, 133, 207-219.

Dafters, R.I., Duffy, F., O'Donnell, P.J. \& Bouquet, C. (1999) Level of use of 3,4-methylenedioxymethamphetamine (MDMA or Ecstasy) in humans correlates with EEG power and coherence. Psychopharmacology, 145, 82-90.

Dalley, J.W., Laane, K., Theobald, D.E., Pena, Y., Bruce, C.C., Huszar, A.C., Wojcieszek, M., Everitt, B.J. \& Robbins, T.W. (2007) Enduring deficits in sustained visual attention during withdrawal of intravenous methylenedioxymethamphetamine self-administration in rats: results from a comparative study with d-amphetamine and methamphetamine. Neuropsychopharmacology, 32, 1195-1206.

Daniela, E., Brennan, K., Gittings, D., Hely, L. \& Schenk, S. (2004) Effect of $\mathrm{SCH} 23390$ on (+/-)-3,4-methylenedioxymethamphetamine hyperactivity and self-administration in rats. Pharmacology, biochemistry, and behavior, 77, 745-750.

Daniela, E., Gittings, D. \& Schenk, S. (2006) Conditioning following repeated exposure to MDMA in rats: role in the maintenance of MDMA self-administration. Behavioral neuroscience, 120, 11441150 .

Darvesh, A.S. \& Gudelsky, G.A. (2004) The relationship between hyperthermia and glycogenolysis in 3,4methylenedioxymethamphetamine-induced serotonin depletion in rats. Neurotoxicology and teratology, 26, 571-577.

Daumann, J., Fischermann, T., Pilatus, U., Thron, A., Moeller-Hartmann, W. \& Gouzoulis-Mayfrank, E. (2004) Proton magnetic resonance spectroscopy in ecstasy (MDMA) users. Neuroscience letters, 362, 113-116.

De La Garza, R., 2nd, Fabrizio, K.R. \& Gupta, A. (2007) Relevance of rodent models of intravenous MDMA self-administration to human MDMA consumption patterns. Psychopharmacology, 189, 425-434.

De la Torre, R., Farre, M., Roset, P.N., Lopez, C.H., Mas, M., Ortuno, J., Menoyo, E., Pizarro, N., Segura, J. \& Cami, J. (2000) Pharmacology of MDMA in humans. Annals of the New York Academy of Sciences, 914, 225-237. 
De Sola Llopis S, Miguelez-Pan, M., Pena-Casanova, J., Poudevida S., Farré, M., Pacifici, R., Böhm, P., Abanades S., Verdejo-Garcia, A., Langhor, K., Zuccaro, P., de la torre, r. (2008) Cognitive performance in recreational ecstasy polydrug users: a two-year follow up study. Journal of Psyhopharmacology, 22, 498-510

De Vry, J., Donselaar, I. \& Van Ree, J.M. (1989) Intraventricular selfadministration of heroin in the rat: reward seems dissociated from analgesia and physical dependence. European journal of pharmacology, 161, 19-25.

de Win, M.M., Jager, G., Booij, J., Reneman, L., Schilt, T., Lavini, C., Olabarriaga, S.D., den Heeten, G.J. \& van den Brink, W. (2008) Sustained effects of ecstasy on the human brain: a prospective neuroimaging study in novel users. Brain : a journal of neurology, 131, 2936-2945.

de Win, M.M., Jager, G., Booij, J., Reneman, L., Schilt, T., Lavini, C., Olabarriaga, S.D., Ramsey, N.F., Heeten, G.J. \& van den Brink, W. (2008) Neurotoxic effects of ecstasy on the thalamus. The British journal of psychiatry : the journal of mental science, 193, 289-296.

Degenhardt, L., Bruno, R. \& Topp, L. (2010) Is ecstasy a drug of dependence? Drug and alcohol dependence, 107, 1-10.

Deneau, G., Yanagita, T. \& Seevers, M.H. (1969) Self-administration of psychoactive substances by the monkey. Psychopharmacologia, 16, $30-48$.

Di Ciano, P., Blaha, C.D. \& Phillips, A.G. (1998) Conditioned changes in dopamine oxidation currents in the nucleus accumbens of rats by stimuli paired with self-administration or yoked-administration of damphetamine. The European journal of neuroscience, 10, 11211127.

Diamond, A. (1995) Evidence of robust recognition memory early in life even when assessed by reaching behavior. Journal of experimental child psychology, 59, 419-456.

Do, J. \& Schenk, S. (2011) Self-administered MDMA produces dose- and time-dependent serotonin deficits in the rat brain. Addiction biology.

Donny, E.C., Caggiula, A.R., Mielke, M.M., Jacobs, K.S., Rose, C. \& Sved, A.F. (1998) Acquisition of nicotine self-administration in rats: the effects of dose, feeding schedule, and drug contingency. Psychopharmacology, 136, 83-90.

Dowling, G.P., McDonough, E.T., 3rd \& Bost, R.O. (1987) 'Eve' and 'Ecstasy'. A report of five deaths associated with the use of MDEA and MDMA. Jama, 257, 1615-1617. 
Dudish, S.A., Pentel, P.R. \& Hatsukami, D.K. (1996) Smoked cocaine selfadministration in females. Psychopharmacology, 123, 79-87.

Durkin, S., Prendergast, A. \& Harkin, A. (2008) Reduced efficacy of fluoxetine following MDMA ("Ecstasy")-induced serotonin loss in rats. Progress in neuro-psychopharmacology \& biological psychiatry, 32, 1894-1901.

Edwards, S., Graham, D.L., Bachtell, R.K. \& Self, D.W. (2007) Regionspecific tolerance to cocaine-regulated cAMP-dependent protein phosphorylation following chronic self-administration. The European journal of neuroscience, 25, 2201-2213.

Egashira, T., Takayama, F. \& Yamanaka, Y. (1999) Changes in monoamine metabolites concentrations in rat cerebrospinal fluid after acute and long-term administration of a selective serotonin reuptake inhibitor, trazodone. Pharmacological research : the official journal of the Italian Pharmacological Society, 40, 503-508.

Ennaceur, A. (1998) Effects of lesions of the Substantia Innominata/Ventral Pallidum, globus pallidus and medial septum on rat's performance in object-recognition and radial-maze tasks: physostigmine and amphetamine treatments. Pharmacological research : the official journal of the Italian Pharmacological Society, 38, 251-263.

Ennaceur, A. (2010) One-trial object recognition in rats and mice: methodological and theoretical issues. Behavioural brain research, 215, 244-254.

Ennaceur, A. \& Delacour, J. (1988) A new one-trial test for neurobiological studies of memory in rats. 1: Behavioral data. Behavioural brain research, 31, 47-59.

Ennaceur, A., Michalikova, S., Bradford, A. \& Ahmed, S. (2005) Detailed analysis of the behavior of Lister and Wistar rats in anxiety, object recognition and object location tasks. Behavioural brain research, 159, 247-266.

Esteban, B., O'Shea, E., Camarero, J., Sanchez, V., Green, A.R. \& Colado, M.I. (2001) 3,4-Methylenedioxymethamphetamine induces monoamine release, but not toxicity, when administered centrally at a concentration occurring following a peripherally injected neurotoxic dose. Psychopharmacology, 154, 251-260.

Esteban, S., Garau, C., Aparicio, S., Moranta, D., Barcelo, P., Fiol, M.A. \& Rial, R. (2010) Chronic melatonin treatment and its precursor Ltryptophan improve the monoaminergic neurotransmission and related behavior in the aged rat brain. Journal of pineal research, $\mathbf{4 8}$, 170-177. 
Esteban, S., Moranta, D., Sastre-Coll, A., Miralles, A. \& Garcia-Sevilla, J.A. (2002) Withdrawal from chronic ethanol increases the sensitivity of presynaptic 5-HT(1A) receptors modulating serotonin and dopamine synthesis in rat brain in vivo. Neuroscience letters, 326, 121-124.

Esteban, S., Nicolaus, C., Garmundi, A., Rial, R.V., Rodriguez, A.B., Ortega, E. \& Ibars, C.B. (2004) Effect of orally administered Ltryptophan on serotonin, melatonin, and the innate immune response in the rat. Molecular and cellular biochemistry, 267, 39-46.

Fagan, J.F., 3rd (1970) Memory in the infant. Journal of experimental child psychology, 9, 217-226.

Fagan, J.F., 3rd (1974) Infant recognition memory: the effects of length of familiarization and type of discrimination task. Child development, 45, 351-356.

Fallon, J.K., Kicman, A.T., Henry, J.A., Milligan, P.J., Cowan, D.A. \& Hutt, A.J. (1999) Stereospecific analysis and enantiomeric disposition of 3, 4-methylenedioxymethamphetamine (Ecstasy) in humans. Clinical chemistry, 45, 1058-1069.

Fantegrossi, W.E., Ullrich, T., Rice, K.C., Woods, J.H. \& Winger, G. (2002) 3,4-Methylenedioxymethamphetamine (MDMA, "ecstasy") and its stereoisomers as reinforcers in rhesus monkeys: serotonergic involvement. Psychopharmacology, 161, 356-364.

Fantegrossi, W.E., Woolverton, W.L., Kilbourn, M., Sherman, P., Yuan, J., Hatzidimitriou, G., Ricaurte, G.A., Woods, J.H. \& Winger, G. (2004) Behavioral and neurochemical consequences of long-term intravenous self-administration of MDMA and its enantiomers by rhesus monkeys. Neuropsychopharmacology, 29, 1270-1281.

Fantegrossi, W.E., Woolverton, W.L., Kilbourn, M., Sherman, P., Yuan, J., Hatzidimitriou, G., Ricaurte, G.A., Woods, J.H. \& Winger, G. (2004) Behavioral and neurochemical consequences of long-term intravenous self-administration of MDMA and its enantiomers by rhesus monkeys. Neuropsychopharmacology, 29, 1270-1281.

Fantegrossi, W.E., Woolverton, W.L., Kilbourn, M., Sherman, P., Yuan, J., Hatzidimitriou, G., Ricaurte, G.A., Woods, J.H. \& Winger, G. (2004) Behavioral and neurochemical consequences of long-term intravenous self-administration of MDMA and its enantiomers by rhesus monkeys. Neuropsychopharmacology, 29, 1270-1281.

Farfel, G.M. \& Seiden, L.S. (1995) Role of hypothermia in the mechanism of protection against serotonergic toxicity. I. Experiments using 3,4methylenedioxymethamphetamine, dizocilpine, CGS 19755 and 
NBQX. The Journal of pharmacology and experimental therapeutics, 272, 860-867.

Farfel, G.M. \& Seiden, L.S. (1995) Role of hypothermia in the mechanism of protection against serotonergic toxicity. I. Experiments using 3,4methylenedioxymethamphetamine, dizocilpine, CGS 19755 and NBQX. The Journal of pharmacology and experimental therapeutics, 272, 860-867.

Farre, M., Abanades, S., Roset, P.N., Peiro, A.M., Torrens, M., O'Mathuna, B., Segura, M. \& de la Torre, R. (2007) Pharmacological interaction between 3,4-methylenedioxymethamphetamine (ecstasy) and paroxetine: pharmacological effects and pharmacokinetics. The Journal of pharmacology and experimental therapeutics, 323, 954962.

Farre, M., de la Torre, R., Mathuna, B.O., Roset, P.N., Peiro, A.M., Torrens, M., Ortuno, J., Pujadas, M. \& Cami, J. (2004) Repeated doses administration of MDMA in humans: pharmacological effects and pharmacokinetics. Psychopharmacology, 173, 364-375.

Feduccia, A.A., Kongovi, N. \& Duvauchelle, C.L. (2010) Heat increases MDMA-enhanced NAcc 5-HT and body temperature, but not MDMA self-administration. European neuropsychopharmacology : the journal of the European College of Neuropsychopharmacology, 20, 884-894.

Fischer, C., Hatzidimitriou, G., Wlos, J., Katz, J. \& Ricaurte, G. (1995) Reorganization of ascending 5-HT axon projections in animals previously exposed to the recreational drug (+/-)3,4methylenedioxymethamphetamine (MDMA, "ecstasy"). J Neurosci, 15, 5476-5485.

Fischer, C., Hatzidimitriou, G., Wlos, J., Katz, J. \& Ricaurte, G. (1995) Reorganization of ascending 5-HT axon projections in animals previously exposed to the recreational drug (+/-)3,4methylenedioxymethamphetamine (MDMA, "ecstasy"). J Neurosci, 15, 5476-5485.

Fisk, J.E. \& Montgomery, C. (2009) Sleep impairment in ecstasy/polydrug and cannabis-only users. The American journal on addictions / American Academy of Psychiatrists in Alcoholism and Addictions, 18, 430-437.

Fitzgerald, J.L. \& Reid, J.J. (1990) Effects of methylenedioxymethamphetamine on the release of monoamines from rat brain slices. European journal of pharmacology, 191, 217220. 
Fitzgerald, J.L. \& Reid, J.J. (1993) Interactions of methylenedioxymethamphetamine with monoamine transmitter release mechanisms in rat brain slices. Naunyn-Schmiedeberg's archives of pharmacology, 347, 313-323.

Foltin, R.W. \& Fischman, M.W. (1992) The cardiovascular and subjective effects of intravenous cocaine and morphine combinations in humans. The Journal of pharmacology and experimental therapeutics, 261, 623-632.

Fox, H.C., Parrott, A.C. \& Turner, J.J. (2001) Ecstasy use: cognitive deficits related to dosage rather than self-reported problematic use of the drug. J Psychopharmacol, 15, 273-281.

Frankel, P.S., Hoonakker, A.J. \& Hanson, G.R. (2008) Differential response of neurotensin to methamphetamine self-administration. Annals of the New York Academy of Sciences, 1139, 112-117.

Frederick, D.L., Ali, S.F., Slikker, W., Jr., Gillam, M.P., Allen, R.R. \& Paule, M.G. (1995) Behavioral and neurochemical effects of chronic methylenedioxymethamphetamine (MDMA) treatment in rhesus monkeys. Neurotoxicology and teratology, 17, 531-543.

Frederick, D.L., Ali, S.F., Slikker, W., Jr., Gillam, M.P., Allen, R.R. \& Paule, M.G. (1995) Behavioral and neurochemical effects of chronic methylenedioxymethamphetamine (MDMA) treatment in rhesus monkeys. Neurotoxicology and teratology, 17, 531-543.

Freezer, A., Salem, A. \& Irvine, R.J. (2005) Effects of 3,4methylenedioxymethamphetamine (MDMA, 'Ecstasy') and paramethoxyamphetamine on striatal 5-HT when co-administered with moclobemide. Brain research, 1041, 48-55.

Freudenmann, R.W., Oxler, F. \& Bernschneider-Reif, S. (2006) The origin of MDMA (ecstasy) revisited: the true story reconstructed from the original documents. Addiction, 101, 1241-1245.

Gamma, A., Buck, A., Berthold, T. \& Vollenweider, F.X. (2001) No difference in brain activation during cognitive performance between ecstasy (3,4-methylenedioxymethamphetamine) users and control subjects: a [H2(15)O]-positron emission tomography study. Journal of clinical psychopharmacology, 21, 66-71.

Gamma, A., Frei, E., Lehmann, D., Pascual-Marqui, R.D., Hell, D. \& Vollenweider, F.X. (2000) Mood state and brain electric activity in ecstasy users. Neuroreport, 11, 157-162.

Garcia-Moreno, L.M., Conejo, N.M., Capilla, A., Garcia-Sanchez, O., Senderek, K. \& Arias, J.L. (2002) Chronic ethanol intake and object 
recognition in young and adult rats. Progress in neuropsychopharmacology \& biological psychiatry, 26, 831-837.

Garcia-Osta, A., Del Rio, J. \& Frechilla, D. (2004) Increased CRE-binding activity and tryptophan hydroxylase mRNA expression induced by 3,4-methylenedioxymethamphetamine (MDMA, "ecstasy") in the rat frontal cortex but not in the hippocampus. Brain research. Molecular brain research, 126, 181-187.

Garcia-Osta, A., Del Rio, J. \& Frechilla, D. (2004) Increased CRE-binding activity and tryptophan hydroxylase mRNA expression induced by 3,4-methylenedioxymethamphetamine (MDMA, "ecstasy") in the rat frontal cortex but not in the hippocampus. Brain research. Molecular brain research, 126, 181-187.

Gartside, S.E., Cowen, P.J. \& Sharp, T. (1992) Effect of 5-hydroxy-Ltryptophan on the release of 5-HT in rat hypothalamus in vivo as measured by microdialysis. Neuropharmacology, 31, 9-14.

Gerber, G.J. \& Stretch, R. (1975) Drug-induced reinstatement of extinguished self-administration behavior in monkeys. Pharmacology, biochemistry, and behavior, 3, 1055-1061.

Gerra, G., Zaimovic, A., Ferri, M., Zambelli, U., Timpano, M., Neri, E., Marzocchi, G.F., Delsignore, R. \& Brambilla, F. (2000) Longlasting effects of (+/-)3,4-methylenedioxymethamphetamine (ecstasy) on serotonin system function in humans. Biological psychiatry, 47, 127-136.

Gerra, G., Zaimovic, A., Giucastro, G., Maestri, D., Monica, C., Sartori, R., Caccavari, R. \& Delsignore, R. (1998) Serotonergic function after (+/-)3,4-methylene-dioxymethamphetamine ('Ecstasy') in humans. International clinical psychopharmacology, 13, 1-9.

Ghanbari, R., El Mansari, M. \& Blier, P. (2010) Sustained administration of trazodone enhances serotonergic neurotransmission: in vivo electrophysiological study in the rat brain. The Journal of pharmacology and experimental therapeutics, 335, 197-206.

Giovannini, M.G., Bartolini, L., Bacciottini, L., Greco, L. \& Blandina, P. (1999) Effects of histamine H3 receptor agonists and antagonists on cognitive performance and scopolamine-induced amnesia. Behavioural brain research, 104, 147-155.

Goldberg, S.R., Hoffmeister, F., Schlichting, U.U. \& Wuttke, W. (1971) A comparison of pentobarbital and cocaine self-administration in rhesus monkeys: effects of dose and fixed-ratio parameter. The Journal of pharmacology and experimental therapeutics, 179, 277283. 
Goni-Allo, B., B, O.M., Segura, M., Puerta, E., Lasheras, B., de la Torre, R. \& Aguirre, N. (2008) The relationship between core body temperature and 3,4-methylenedioxymethamphetamine metabolism in rats: implications for neurotoxicity. Psychopharmacology, 197, 263-278.

Gonzalez-Burgos, I. \& Feria-Velasco, A. (2008) Serotonin/dopamine interaction in memory formation. Progress in brain research, 172, 603-623.

Gough, B., Ali, S.F., Slikker, W., Jr. \& Holson, R.R. (1991) Acute effects of 3,4-methylenedioxymethamphetamine (MDMA) on monoamines in rat caudate. Pharmacology, biochemistry, and behavior, 39, 619-623.

Gould, R.W., Gage, H.D., Banks, M.L., Blaylock, B.L., Czoty, P.W. \& Nader, M.A. (2011) Differential effects of cocaine and MDMA selfadministration on cortical serotonin transporter availability in monkeys. Neuropharmacology, 61, 245-251.

Gouzoulis-Mayfrank, E. \& Daumann, J. (2006) The confounding problem of polydrug use in recreational ecstasy/MDMA users: a brief overview. J Psychopharmacol, 20, 188-193.

Gouzoulis-Mayfrank, E. \& Daumann, J. (2006) Neurotoxicity of methylenedioxyamphetamines (MDMA; ecstasy) in humans: how strong is the evidence for persistent brain damage? Addiction, 101, 348-361.

Gouzoulis-Mayfrank, E., Fischermann, T., Rezk, M., Thimm, B., Hensen, G. \& Daumann, J. (2005) Memory performance in polyvalent MDMA (ecstasy) users who continue or discontinue MDMA use. Drug and alcohol dependence, 78, 317-323.

Gouzoulis-Mayfrank, E., Thimm, B., Rezk, M., Hensen, G. \& Daumann, J. (2003) Memory impairment suggests hippocampal dysfunction in abstinent ecstasy users. Progress in neuro-psychopharmacology \& biological psychiatry, 27, 819-827.

Grahame, N.J. \& Cunningham, C.L. (1995) Genetic differences in intravenous cocaine self-administration between C57BL/6J and DBA/2J mice. Psychopharmacology, 122, 281-291.

Granoff, M.I. \& Ashby, C.R., Jr. (2001) Effect of the repeated administration of (+/-)-3,4-methylenedioxymethamphetamine on the behavioral response of rats to the 5-HT1A receptor agonist (+/-)-8hydroxy-(di-n-propylamino)tetralin. Neuropsychobiology, 43, 42-48.

Green, A.R., Gabrielsson, J., Marsden, C.A. \& Fone, K.C. (2009) MDMA: on the translation from rodent to human dosing. Psychopharmacology, 204, 375-378. 
Green, A.R., Mechan, A.O., Elliott, J.M., O'Shea, E. \& Colado, M.I. (2003) The pharmacology and clinical pharmacology of 3,4methylenedioxymethamphetamine (MDMA, "ecstasy"). Pharmacological reviews, 55, 463-508.

Griffiths, R.R., Lukas, S.E., Bradford, L.D., Brady, J.V. \& Snell, J.D. (1981) Self-injection of barbiturates and benzodiazepines in baboons. Psychopharmacology, 75, 101-109.

Grove, R.N. \& Schuster, C.R. (1974) Suppression of cocaine selfadministration by extinction and punishment. Pharmacology, biochemistry, and behavior, 2, 199-208.

Gu, X.F. \& Azmitia, E.C. (1993) Integrative transporter-mediated release from cytoplasmic and vesicular 5-hydroxytryptamine stores in cultured neurons. European journal of pharmacology, 235, 51-57.

Gu, X.F. \& Azmitia, E.C. (1993) Integrative transporter-mediated release from cytoplasmic and vesicular 5-hydroxytryptamine stores in cultured neurons. European journal of pharmacology, 235, 51-57.

Gudelsky, G.A. \& Nash, J.F. (1996) Carrier-mediated release of serotonin by 3,4-methylenedioxymethamphetamine: implications for serotonin-dopamine interactions. Journal of neurochemistry, 66 , 243-249.

Haddjeri, N., Lavoie, N. \& Blier, P. (2004) Electrophysiological evidence for the tonic activation of 5-HT(1A) autoreceptors in the rat dorsal raphe nucleus. Neuropsychopharmacology, 29, 1800-1806.

Hadjiefthyvoulou, F., Fisk, J.E., Montgomery, C. \& Bridges, N. (2011) Prospective memory functioning among ecstasy/polydrug users: evidence from the Cambridge Prospective Memory Test (CAMPROMPT). Psychopharmacology, 215, 761-774.

Haider, S., Khaliq, S., Ahmed, S.P. \& Haleem, D.J. (2006) Long-term tryptophan administration enhances cognitive performance and increases 5HT metabolism in the hippocampus of female rats. Amino acids, 31, 421-425.

Haider, S., Khaliq, S. \& Haleem, D.J. (2007) Enhanced serotonergic neurotransmission in the hippocampus following tryptophan administration improves learning acquisition and memory consolidation in rats. Pharmacological reports : $P R$, 59, 53-57.

Hamon, M., Fattaccini, C.M., Adrien, J., Gallissot, M.C., Martin, P. \& Gozlan, H. (1988) Alterations of central serotonin and dopamine turnover in rats treated with ipsapirone and other 5hydroxytryptamine1A agonists with potential anxiolytic properties. 
The Journal of pharmacology and experimental therapeutics, 246, 745-752.

Hansen, F., de Oliveira, D.L., Amaral, F.U., Guedes, F.S., Schneider, T.J., Tumelero, A.C., Hansel, G., Schmidt, K.H., Giacomini, A.C. \& Torres, F.V. (2011) Effects of chronic administration of tryptophan with or without concomitant fluoxetine in depression-related and anxiety-like behaviors on adult rat. Neuroscience letters, 499, 59-63.

Hanson, G.R., Hoonakker, A.J., Alburges, M.E., McFadden, L.M., Robson, C.M. \& Frankel, P.S. (2012) Response of limbic neurotensin systems to methamphetamine self-administration. Neuroscience, 203, 99-107.

Hanson, K.L., Luciana, M. \& Sullwold, K. (2008) Reward-related decisionmaking deficits and elevated impulsivity among MDMA and other drug users. Drug and alcohol dependence, 96, 99-110.

Harper, D.N., Wisnewski, R., Hunt, M. \& Schenk, S. (2005) (+/-)3,4methylenedioxymethamphetamine, d-amphetamine, and cocaine impair delayed matching-to-sample performance by an increase in susceptibility to proactive interference. Behavioral neuroscience, 119, 455-463.

Hayakawa, T., Shibata, K. \& Iwai, K. (1991) Why does a difference in growth rate between rats of the Wistar and Sprague Dawley strains occur when a niacin-free and tryptophan-limiting diet is fed? Advances in experimental medicine and biology, 294, 649-651.

He, J., Yang, Y., Yu, Y., Li, X. \& Li, X.M. (2006) The effects of chronic administration of quetiapine on the methamphetamine-induced recognition memory impairment and dopaminergic terminal deficit in rats. Behavioural brain research, 172, 39-45.

Heffner, T.G., Hartman, J.A. \& Seiden, L.S. (1980) A rapid method for the regional dissection of the rat brain. Pharmacology, biochemistry, and behavior, 13, 453-456.

Heffner, T.G., Hartman, J.A. \& Seiden, L.S. (1980) A rapid method for the regional dissection of the rat brain. Pharmacology, biochemistry, and behavior, 13, 453-456.

Hekmatpanah, C.R. \& Peroutka, S.J. (1990) 5-hydroxytryptamine uptake blockers attenuate the 5-hydroxytryptamine-releasing effect of 3,4methylenedioxymethamphetamine and related agents. European journal of pharmacology, 177, 95-98.

Herring, N.R., Schaefer, T.L., Gudelsky, G.A., Vorhees, C.V. \& Williams, M.T. (2008) Effect of +-methamphetamine on path integration 
learning, novel object recognition, and neurotoxicity in rats. Psychopharmacology, 199, 637-650.

Hewton, R., Salem, A. \& Irvine, R.J. (2007) Potentiation of 3,4methylenedioxymethamphetamine-induced 5-HT release in the rat substantia nigra by clorgyline, a monoamine oxidase A inhibitor. Clinical and experimental pharmacology \& physiology, 34, 10511057.

Heydari, A., Yeo, K.R., Lennard, M.S., Ellis, S.W., Tucker, G.T. \& Rostami-Hodjegan, A. (2004) Mechanism-based inactivation of CYP2D6 by methylenedioxymethamphetamine. Drug metabolism and disposition: the biological fate of chemicals, 32, 1213-1217.

Heyser, C.J. \& Chemero, A. (2012) Novel object exploration in mice: Not all objects are created equal. Behavioural processes, 89, 232-238.

Hicks, A.A., Pramstaller, P.P., Johansson, A., Vitart, V., Rudan, I., Ugocsai, P., Aulchenko, Y., Franklin, C.S., Liebisch, G., Erdmann, J., Jonasson, I., Zorkoltseva, I.V., Pattaro, C., Hayward, C., Isaacs, A., Hengstenberg, C., Campbell, S., Gnewuch, C., Janssens, A.C., Kirichenko, A.V., Konig, I.R., Marroni, F., Polasek, O., Demirkan, A., Kolcic, I., Schwienbacher, C., Igl, W., Biloglav, Z., Witteman, J.C., Pichler, I., Zaboli, G., Axenovich, T.I., Peters, A., Schreiber, S., Wichmann, H.E., Schunkert, H., Hastie, N., Oostra, B.A., Wild, S.H., Meitinger, T., Gyllensten, U., van Duijn, C.M., Wilson, J.F., Wright, A., Schmitz, G. \& Campbell, H. (2009) Genetic determinants of circulating sphingolipid concentrations in European populations. PLoS genetics, 5, e1000672.

Hingtgen, J.N., Hendrie, H.C. \& Aprison, M.H. (1984) Postsynaptic serotonergic blockade following chronic antidepressive treatment with trazodone in an animal model of depression. Pharmacology, biochemistry, and behavior, 20, 425-428.

Hiramatsu, M., DiStefano, E., Chang, A.S. \& Cho, A.K. (1991) A pharmacokinetic analysis of 3,4-methylenedioxymethamphetamine effects on monoamine concentrations in brain dialysates. European journal of pharmacology, 204, 135-140.

Hirt, D., Fonsart, J., Menet, M.C., Debray, M., Noble, F., Decleves, X. \& Scherrmann, J.M. (2010) Population pharmacokinetics of 3,4methylenedioxymethamphetamine and main metabolites in rats. Toxicological sciences : an official journal of the Society of Toxicology, 114, 38-47.

Hjorth, S., Carlsson A., Lindberg, P., Sanchez, D., Wikström, H., Arvidsson, L.E., Hacksell U. \& Nilson J. (1982) 8-hydroxy-2-(Di-npropylamino) Tetralin, 8-OH-DPAT, a potent and selective 
simplified ergot congener with central 5-HT-receptor stimulating activity. J. Nerual Transmission, 55, 169-188

Hjorth, S. (1996) (-)-Pindolol, but not buspirone, potentiates the citalopraminduced rise in extracellular 5-hydroxytryptamine. European journal of pharmacology, 303, 183-186.

Hjorth, S. \& Magnusson, T. (1988) The 5-HT 1A receptor agonist, 8-OHDPAT, preferentially activates cell body 5 -HT autoreceptors in rat brain in vivo. Naunyn-Schmiedeberg's archives of pharmacology, 338, 463-471.

Hjorth, S. \& Magnusson, T. (1988) The 5-HT 1A receptor agonist, 8-OHDPAT, preferentially activates cell body 5-HT autoreceptors in rat brain in vivo. Naunyn-Schmiedeberg's archives of pharmacology, 338, 463-471.

Hodges, H. (1996) Maze procedures: the radial-arm and water maze compared. Brain research. Cognitive brain research, 3, 167-181.

Hoffmeister, F. \& Goldberg, S.R. (1973) A comparison of chlorpromazine, imipramine, morphine and d-amphetamine self-administration in cocaine-dependent rhesus monkeys. The Journal of pharmacology and experimental therapeutics, 187, 8-14.

Hoffmeister, F., Goldberg, S.R., Schlichting, U. \& Wuttke, W. (1970) Selfadministration of $\mathrm{d}$-amphetamine, morphine and chlorpromazine by cocaine "dependent" rhesus monkeys. Naunyn-Schmiedebergs Archiv fur Pharmakologie, 266, 359-360.

Hoshi, R., Mullins, K., Boundy, C., Brignell, C., Piccini, P. \& Curran, H.V. (2007) Neurocognitive function in current and ex-users of ecstasy in comparison to both matched polydrug-using controls and drug-naive controls. Psychopharmacology, 194, 371-379.

Indlekofer, F., Piechatzek, M., Daamen, M., Glasmacher, C., Lieb, R., Pfister, H., Tucha, O., Lange, K.W., Wittchen, H.U. \& Schutz, C.G. (2009) Reduced memory and attention performance in a populationbased sample of young adults with a moderate lifetime use of cannabis, ecstasy and alcohol. J Psychopharmacol, 23, 495-509.

Insel, T.R., Battaglia, G., Johannessen, J.N., Marra, S. \& De Souza, E.B. (1989) 3,4-Methylenedioxymethamphetamine ("ecstasy") selectively destroys brain serotonin terminals in rhesus monkeys. The Journal of pharmacology and experimental therapeutics, 249, 713-720.

Jacobs, E.H., de Vries, T.J., Smit, A.B. \& Schoffelmeer, A.N. (2004) Gene transcripts selectively down-regulated in the shell of the nucleus accumbens long after heroin self-administration are up-regulated in the core independent of response contingency. FASEB journal : 
official publication of the Federation of American Societies for Experimental Biology, 18, 200-202.

Jacobs, E.H., Smit, A.B., de Vries, T.J. \& Schoffelmeer, A.N. (2003) Neuroadaptive effects of active versus passive drug administration in addiction research. Trends in pharmacological sciences, 24, 566573.

Jansen, K.L. (1999) Ecstasy (MDMA) dependence. Drug and alcohol dependence, 53, 121-124.

Jenkins, T.A., Elliott, J.J., Ardis, T.C., Cahir, M., Reynolds, G.P., Bell, R. \& Cooper, S.J. (2010) Tryptophan depletion impairs object-recognition memory in the rat: reversal by risperidone. Behavioural brain research, 208, 479-483.

Jensen, K.F., Olin, J., Haykal-Coates, N., O'Callaghan, J., Miller, D.B. \& de Olmos, J.S. (1993) Mapping toxicant-induced nervous system damage with a cupric silver stain: a quantitative analysis of neural degeneration induced by 3,4-methylenedioxymethamphetamine. NIDA research monograph, 136, 133-149; discussion 150-134.

Johnson, M., Elayan, I., Hanson, G.R., Foltz, R.L., Gibb, J.W. \& Lim, H.K. (1992) Effects of 3,4-dihydroxymethamphetamine and 2,4,5trihydroxymethamphetamine, two metabolites of $3,4-$ methylenedioxymethamphetamine, on central serotonergic and dopaminergic systems. The Journal of pharmacology and experimental therapeutics, 261, 447-453.

Johnson, M.P., Hoffman, A.J. \& Nichols, D.E. (1986) Effects of the enantiomers of MDA, MDMA and related analogues on $[3 \mathrm{H}]$ serotonin and $[3 \mathrm{H}]$ dopamine release from superfused rat brain slices. European journal of pharmacology, 132, 269-276.

Johnson, R.G., Fiorella, D., Winter, J.C. \& Rabin, R.A. (1997) [3H]8-OHDPAT labels a 5-HT site coupled to inhibition of phosphoinositide hydrolysis in the dorsal raphe. European journal of pharmacology, 329, 99-106.

Kalechstein, A.D., De La Garza, R., 2nd, Mahoney, J.J., 3rd, Fantegrossi, W.E. \& Newton, T.F. (2007) MDMA use and neurocognition: a meta-analytic review. Psychopharmacology, 189, 531-537.

Katayama, J., Yakushiji, T. \& Akaike, N. (1997) Characterization of the K+ current mediated by 5 -HT1A receptor in the acutely dissociated rat dorsal raphe neurons. Brain research, 745, 283-292.

Kay, C., Harper, D.N. \& Hunt, M. (2010) Differential effects of MDMA and scopolamine on working versus reference memory in the radial arm maze task. Neurobiology of learning and memory, 93, 151-156. 
Kay, C., Harper, D.N. \& Hunt, M. (2011) The effects of binge MDMA on acquisition and reversal learning in a radial-arm maze task. Neurobiology of learning and memory, 95, 473-483.

Kehr, J., Ichinose, F., Yoshitake, S., Goiny, M., Sievertsson, T., Nyberg, F. \& Yoshitake, T. (2011) Mephedrone, compared with MDMA (ecstasy) and amphetamine, rapidly increases both dopamine and 5HT levels in nucleus accumbens of awake rats. British journal of pharmacology, 164, 1949-1958.

Kelai, S., Renoir, T., Chouchana, L., Saurini, F., Hanoun, N., Hamon, M. \& Lanfumey, L. (2008) Chronic voluntary ethanol intake hypersensitizes 5-HT(1A) autoreceptors in C57BL/6J mice. Journal of neurochemistry, 107, 1660-1670.

Kelai, S., Renoir, T., Chouchana, L., Saurini, F., Hanoun, N., Hamon, M. \& Lanfumey, L. (2008) Chronic voluntary ethanol intake hypersensitizes 5-HT(1A) autoreceptors in C57BL/6J mice. Journal of neurochemistry, 107, 1660-1670.

Khaliq, S., Haider, S. \& Haleem, D.J. (2007) Comparative effects of single dose and repeated oral tryptophan administration on indoleamine synthesis and memory functions in rats. Pakistan journal of pharmaceutical sciences, 20, 71-76.

Kilbey, M.M. \& Ellinwood, E.H., Jr. (1980) Self-administration of morphine in the cat. The International journal of the addictions, 15, 447-460.

Kim, C.K., Kalynchuk, L.E., Kornecook, T.J., Mumby, D.G., Dadgar, N.A., Pinel, J.P. \& Weinberg, J. (1997) Object-recognition and spatial learning and memory in rats prenatally exposed to ethanol. Behavioral neuroscience, 111, 985-995.

King, M.V., Spicer, C.H., Sleight, A.J., Marsden, C.A. \& Fone, K.C. (2009) Impact of regional 5-HT depletion on the cognitive enhancing effects of a typical 5-ht(6) receptor antagonist, Ro 04-6790, in the Novel Object Discrimination task. Psychopharmacology, 202, 111123.

Kish, S.J., Fitzmaurice, P.S., Chang, L.J., Furukawa, Y. \& Tong, J. (2010) Low striatal serotonin transporter protein in a human polydrug MDMA (ecstasy) user: a case study. J Psychopharmacol, 24, 281284.

Kittler, K., Lau, T. \& Schloss, P. (2010) Antagonists and substrates differentially regulate serotonin transporter cell surface expression in serotonergic neurons. European journal of pharmacology, 629, 6367. 
Kivell, B., Day, D., Bosch, P., Schenk, S. \& Miller, J. (2010) MDMA causes a redistribution of serotonin transporter from the cell surface to the intracellular compartment by a mechanism independent of phospho-p38-mitogen activated protein kinase activation. Neuroscience, 168, 82-95.

Kleven, M., Ybema, C., Carilla, E., Hamon, M. \& Koek, W. (1995) Modification of behavioral effects of 8-hydroxy-2-(di-npropylamino)tetralin following chronic ethanol consumption in the rat: evidence for the involvement of 5-HT1A receptors in ethanol dependence. European journal of pharmacology, 281, 219-228.

Kleven, M., Ybema, C., Carilla, E., Hamon, M. \& Koek, W. (1995) Modification of behavioral effects of 8-hydroxy-2-(di-npropylamino)tetralin following chronic ethanol consumption in the rat: evidence for the involvement of 5-HT1A receptors in ethanol dependence. European journal of pharmacology, 281, 219-228.

Koek, W., Assie, M.B., Zernig, G. \& France, C.P. (2000) In vivo estimates of efficacy at 5-HT1A receptors: effects of EEDQ on the ability of agonists to produce lower-lip retraction in rats. Psychopharmacology, 149, 377-387.

Koek, W., Patoiseau, J.F., Assie, M.B., Cosi, C., Kleven, M.S., DupontPasselaigue, E., Carilla-Durand, E., Palmier, C., Valentin, J.P., John, G., Pauwels, P.J., Tarayre, J.P. \& Colpaert, F.C. (1998) F 11440, a potent, selective, high efficacy 5-HT1A receptor agonist with marked anxiolytic and antidepressant potential. The Journal of pharmacology and experimental therapeutics, 287, 266-283.

Kolbrich, E.A., Goodwin, R.S., Gorelick, D.A., Hayes, R.J., Stein, E.A. \& Huestis, M.A. (2008) Plasma pharmacokinetics of 3,4methylenedioxymethamphetamine after controlled oral administration to young adults. Therapeutic drug monitoring, 30, 320-332.

Kosiorek, P., Hryniewicz, A., Bialuk, I., Zawadzka, A. \& Winnicka, M.M. (2003) Cannabinoids alter recognition memory in rats. Polish journal of pharmacology, 55, 903-910.

Kovacs, G.G., Ando, R.D., Adori, C., Kirilly, E., Benedek, A., Palkovits, M. \& Bagdy, G. (2007) Single dose of MDMA causes extensive decrement of serotoninergic fibre density without blockage of the fast axonal transport in Dark Agouti rat brain and spinal cord. Neuropathology and applied neurobiology, 33, 193-203.

Kovacs, G.G., Ando, R.D., Adori, C., Kirilly, E., Benedek, A., Palkovits, M. \& Bagdy, G. (2007) Single dose of MDMA causes extensive decrement of serotoninergic fibre density without blockage of the 
fast axonal transport in Dark Agouti rat brain and spinal cord. Neuropathology and applied neurobiology, 33, 193-203.

Kreiss, D.S. \& Lucki, I. (1992) Desensitization of 5-HT1A autoreceptors by chronic administration of 8-OH-DPAT. Neuropharmacology, 31, 1073-1076.

Krystal, J.H., Price, L.H., Opsahl, C., Ricaurte, G.A. \& Heninger, G.R. (1992) Chronic 3,4-methylenedioxymethamphetamine (MDMA) use: effects on mood and neuropsychological function? The American journal of drug and alcohol abuse, 18, 331-341.

Kumagai, Y., Lin, L.Y., Hiratsuka, A., Narimatsu, S., Suzuki, T., Yamada, H., Oguri, K., Yoshimura, H. \& Cho, A.K. (1994) Participation of cytochrome P450-2B and -2D isozymes in the demethylenation of methylenedioxymethamphetamine enantiomers by rats. Molecular pharmacology, 45, 359-365.

Kuzmin, A., Johansson, B., Semenova, S. \& Fredholm, B.B. (2000) Differences in the effect of chronic and acute caffeine on selfadministration of cocaine in mice. The European journal of neuroscience, 12, 3026-3032.

Lamb, R.J. \& McMillan, D.E. (1986) The effects of some putative antidepressant agents on the schedule-controlled behavior of the pigeon. Psychopharmacology, 88, 368-373.

Lau, C.E. \& Sun, L. (2002) The pharmacokinetic determinants of the frequency and pattern of intravenous cocaine self-administration in rats by pharmacokinetic modeling. Drug metabolism and disposition: the biological fate of chemicals, 30, 254-261.

Laws, K.R. \& Kokkalis, J. (2007) Ecstasy (MDMA) and memory function: a meta-analytic update. Human psychopharmacology, 22, 381-388.

Lebrun, C., Pilliere, E. \& Lestage, P. (2000) Effects of S 18986-1, a novel cognitive enhancer, on memory performances in an object recognition task in rats. European journal of pharmacology, 401, 205-212.

Lehmann, J., DeSouza, E.B., Culp, S. \& Zaczek, R. (1992) Regional distribution to recovery of 5-HT levels after administration of "atrophins" MDMA and D,L-fenfluramine. Stereospecificity and comparison with 5,7-dihydroxytryptamine. Annals of the New York Academy of Sciences, 648, 291-295.

Lew, R., Sabol, K.E., Chou, C., Vosmer, G.L., Richards, J. \& Seiden, L.S. (1996) Methylenedioxymethamphetamine-induced serotonin deficits are followed by partial recovery over a 52-week period. Part II: 
Radioligand binding and autoradiography studies. The Journal of pharmacology and experimental therapeutics, 276, 855-865.

Li, J.X. \& France, C.P. (2008) Food restriction and streptozotocin treatment decrease 5-HT1A and 5-HT2A receptor-mediated behavioral effects in rats. Behavioural pharmacology, 19, 292-297.

Lieben, C.K., Steinbusch, H.W. \& Blokland, A. (2006) 5,7-DHT lesion of the dorsal raphe nuclei impairs object recognition but not affective behavior and corticosterone response to stressor in the rat. Behavioural brain research, 168, 197-207.

Lieben, C.K., van Oorsouw, K., Deutz, N.E. \& Blokland, A. (2004) Acute tryptophan depletion induced by a gelatin-based mixture impairs object memory but not affective behavior and spatial learning in the rat. Behavioural brain research, 151, 53-64.

Liechti, M.E., Baumann, C., Gamma, A. \& Vollenweider, F.X. (2000) Acute psychological effects of 3,4methylenedioxymethamphetamine (MDMA, "Ecstasy") are attenuated by the serotonin uptake inhibitor citalopram. Neuropsychopharmacology, 22, 513-521.

Lile, J.A., Ross, J.T. \& Nader, M.A. (2005) A comparison of the reinforcing efficacy of 3,4-methylenedioxymethamphetamine (MDMA, "ecstasy") with cocaine in rhesus monkeys. Drug and alcohol dependence, 78, 135-140.

Ludwig, V., Mihov, Y. \& Schwarting, R.K. (2008) Behavioral and neurochemical consequences of multiple MDMA administrations in the rat: role of individual differences in anxiety-related behavior. Behavioural brain research, 189, 52-64.

MacInnes, N., Handley, S.L. \& Harding, G.F. (2001) Former chronic methylenedioxymethamphetamine (MDMA or ecstasy) users report mild depressive symptoms. J Psychopharmacol, 15, 181-186.

MacKay-Soroka, S., Trehub, S.E., Bull, D.H. \& Corter, C.M. (1982) Effects of encoding and retrieval conditions on infants' recognition memory. Child development, 53, 815-818.

Maj, J., Palider, W. \& Rawlow (1979) Trazodone, a central serotonin antagonist and agonist. J Neural Transm, 44, 237-248.

Malberg, J.E., Sabol, K.E. \& Seiden, L.S. (1996) Co-administration of MDMA with drugs that protect against MDMA neurotoxicity produces different effects on body temperature in the rat. The Journal of pharmacology and experimental therapeutics, 278, 258267. 
Marshall, J.F., Belcher, A.M., Feinstein, E.M. \& O'Dell, S.J. (2007) Methamphetamine-induced neural and cognitive changes in rodents. Addiction, 102 Suppl 1, 61-69.

Marston, H.M., Reid, M.E., Lawrence, J.A., Olverman, H.J. \& Butcher, S.P. (1999) Behavioural analysis of the acute and chronic effects of MDMA treatment in the rat. Psychopharmacology, 144, 67-76.

Martellotta, M.C., Kuzmin, A., Zvartau, E., Cossu, G., Gessa, G.L. \& Fratta, W. (1995) Isradipine inhibits nicotine intravenous selfadministration in drug-naive mice. Pharmacology, biochemistry, and behavior, 52, 271-274.

Martins, S.S., Storr, C.L., Alexandre, P.K. \& Chilcoat, H.D. (2008) Do adolescent ecstasy users have different attitudes towards drugs when compared to marijuana users? Drug and alcohol dependence, 94, 6372 .

Mas, M., Farre, M., de la Torre, R., Roset, P.N., Ortuno, J., Segura, J. \& Cami, J. (1999) Cardiovascular and neuroendocrine effects and pharmacokinetics of 3, 4-methylenedioxymethamphetamine in humans. The Journal of pharmacology and experimental therapeutics, 290, 136-145.

McCann, U.D., Mertl, M., Eligulashvili, V. \& Ricaurte, G.A. (1999) Cognitive performance in (+/-) 3,4methylenedioxymethamphetamine (MDMA, "ecstasy") users: a controlled study. Psychopharmacology, 143, 417-425.

McCann, U.D. \& Ricaurte, G.A. (1991) Lasting neuropsychiatric sequelae of (+-)methylenedioxymethamphetamine ('ecstasy') in recreational users. Journal of clinical psychopharmacology, 11, 302-305.

McCann, U.D. \& Ricaurte, G.A. (1992) MDMA ("ecstasy") and panic disorder: induction by a single dose. Biological psychiatry, 32, 950953.

McCann, U.D., Szabo, Z., Scheffel, U., Dannals, R.F. \& Ricaurte, G.A. (1998) Positron emission tomographic evidence of toxic effect of MDMA ("Ecstasy") on brain serotonin neurons in human beings. Lancet, 352, 1433-1437.

McCann, U.D., Szabo, Z., Seckin, E., Rosenblatt, P., Mathews, W.B., Ravert, H.T., Dannals, R.F. \& Ricaurte, G.A. (2005) Quantitative PET studies of the serotonin transporter in MDMA users and controls using [11C]McN5652 and [11C]DASB. Neuropsychopharmacology, 30, 1741-1750.

McCann, U.D., Szabo, Z., Vranesic, M., Palermo, M., Mathews, W.B., Ravert, H.T., Dannals, R.F. \& Ricaurte, G.A. (2008) Positron 
emission tomographic studies of brain dopamine and serotonin transporters in abstinent (+/-)3,4-methylenedioxymethamphetamine ("ecstasy") users: relationship to cognitive performance. Psychopharmacology, 200, 439-450.

McCardle, K., Luebbers, S., Carter, J.D., Croft, R.J. \& Stough, C. (2004) Chronic MDMA (ecstasy) use, cognition and mood. Psychopharmacology, 173, 434-439.

McCreary, A.C., Bankson, M.G. \& Cunningham, K.A. (1999) Pharmacological studies of the acute and chronic effects of (+)-3, 4methylenedioxymethamphetamine on locomotor activity: role of 5hydroxytryptamine(1A) and 5-hydroxytryptamine(1B/1D) receptors. The Journal of pharmacology and experimental therapeutics, 290, 965-973.

McGregor, I.S., Gurtman, C.G., Morley, K.C., Clemens, K.J., Blokland, A., Li, K.M., Cornish, J.L. \& Hunt, G.E. (2003) Increased anxiety and "depressive" symptoms months after MDMA ("ecstasy") in rats: drug-induced hyperthermia does not predict long-term outcomes. Psychopharmacology, 168, 465-474.

McKenna, D.J., Guan, X.M. \& Shulgin, A.T. (1991) 3,4Methylenedioxyamphetamine (MDA) analogues exhibit differential effects on synaptosomal release of 3H-dopamine and 3H-5hydroxytryptamine. Pharmacology, biochemistry, and behavior, 38, 505-512.

Mechan, A., Yuan, J., Hatzidimitriou, G., Irvine, R.J., McCann, U.D. \& Ricaurte, G.A. (2006) Pharmacokinetic profile of single and repeated oral doses of MDMA in squirrel monkeys: relationship to lasting effects on brain serotonin neurons. Neuropsychopharmacology, 31, 339-350.

Medina, K.L. \& Shear, P.K. (2007) Anxiety, depression, and behavioral symptoms of executive dysfunction in ecstasy users: contributions of polydrug use. Drug and alcohol dependence, 87, 303-311.

Mendelson, W.B. (2005) A review of the evidence for the efficacy and safety of trazodone in insomnia. The Journal of clinical psychiatry, 66, 469-476.

Mendelson, W.B. (2005) A review of the evidence for the efficacy and safety of trazodone in insomnia. The Journal of clinical psychiatry, 66, 469-476.

Meneses, A., Perez-Garcia, G., Ponce-Lopez, T., Tellez, R. \& Castillo, C. (2011) Serotonin transporter and memory. Neuropharmacology, 61, 355-363. 
Metaxas, A., Bailey, A., Barbano, M.F., Galeote, L., Maldonado, R. \& Kitchen, I. (2010) Differential region-specific regulation of alpha4beta2* nAChRs by self-administered and non-contingent nicotine in C57BL/6J mice. Addiction biology, 15, 464-479.

Mierzejewski, P., Stefanski, R., Bienkowski, P. \& Kostowski, W. (2007) History of cocaine self-administration alters morphine reinforcement in the rat. European journal of pharmacology, 562, 77-81.

Milroy, C.M. (1999) Ten years of 'ecstasy'. Journal of the Royal Society of Medicine, 92, 68-72.

Mittur, A. (2011) Trazodone: properties and utility in multiple disorders. Expert review of clinical pharmacology, 4, 181-196.

Moja, E.A., Cipolla, P., Castoldi, D. \& Tofanetti, O. (1989) Dose-response decrease in plasma tryptophan and in brain tryptophan and serotonin after tryptophan-free amino acid mixtures in rats. Life sciences, $\mathbf{4 4 ,}$ 971-976.

Mokler, D.J., Robinson, S.E. \& Rosecrans, J.A. (1987) (+/-)3,4Methylenedioxymethamphetamine (MDMA) produces long-term reductions in brain 5-hydroxytryptamine in rats. European journal of pharmacology, 138, 265-268.

Money, E.A., Kirk, R.C. \& McNaughton, N. (1992) Alzheimer's dementia produces a loss of discrimination but no increase in rate of memory decay in delayed matching to sample. Neuropsychologia, 30, 133143.

Montgomery, C. \& Fisk, J.E. (2007) Everyday memory deficits in ecstasypolydrug users. J Psychopharmacol, 21, 709-717.

Montgomery, C., Fisk, J.E., Wareing, M. \& Murphy, P. (2007) Self reported sleep quality and cognitive performance in ecstasy users. Human psychopharmacology, 22, 537-548.

Morley, K.C., Gallate, J.E., Hunt, G.E., Mallet, P.E. \& McGregor, I.S. (2001) Increased anxiety and impaired memory in rats 3 months after administration of 3,4-methylenedioxymethamphetamine ("ecstasy"). European journal of pharmacology, 433, 91-99.

Morrow, B.A., Elsworth, J.D. \& Roth, R.H. (2002) Prenatal cocaine exposure disrupts non-spatial, short-term memory in adolescent and adult male rats. Behavioural brain research, 129, 217-223.

Morrow, B.A., Roth, R.H. \& Elsworth, J.D. (2000) TMT, a predator odor, elevates mesoprefrontal dopamine metabolic activity and disrupts short-term working memory in the rat. Brain research bulletin, 52, 519-523. 
Mueller, M., Kolbrich, E.A., Peters, F.T., Maurer, H.H., McCann, U.D., Huestis, M.A. \& Ricaurte, G.A. (2009) Direct comparison of (+/-) 3,4-methylenedioxymethamphetamine ("ecstasy") disposition and metabolism in squirrel monkeys and humans. Therapeutic drug monitoring, 31, 367-373.

Mueller, M., Peters, F.T., Maurer, H.H., McCann, U.D. \& Ricaurte, G.A. (2008) Nonlinear pharmacokinetics of (+/-)3,4methylenedioxymethamphetamine (MDMA, "Ecstasy") and its major metabolites in squirrel monkeys at plasma concentrations of MDMA that develop after typical psychoactive doses. The Journal of pharmacology and experimental therapeutics, 327, 38-44.

Murphy, P.N., Wareing, M., Fisk, J.E. \& Montgomery, C. (2009) Executive working memory deficits in abstinent ecstasy/MDMA users: a critical review. Neuropsychobiology, 60, 159-175.

Nash, J.F. \& Nichols, D.E. (1991) Microdialysis studies on 3,4methylenedioxyamphetamine and structurally related analogues. European journal of pharmacology, 200, 53-58.

Nawata, Y., Hiranita, T. \& Yamamoto, T. (2010) A cannabinoid CB(1) receptor antagonist ameliorates impairment of recognition memory on withdrawal from MDMA (Ecstasy). Neuropsychopharmacology, 35, 515-520.

Neckers, L.M., Neff, N.H. \& Wyatt, R.J. (1979) Increased serotonin turnover in corpus striatum following an injection of kainic acid: evidence for neuronal feedback regulation of synthesis. NaunynSchmiedeberg's archives of pharmacology, 306, 173-177.

Nevo, I., Langlois, X., Laporte, A.M., Kleven, M., Koek, W., Lima, L., Maudhuit, C., Martres, M.P. \& Hamon, M. (1995) Chronic alcoholization alters the expression of 5-HT1A and 5-HT1B receptor subtypes in rat brain. European journal of pharmacology, 281, 229239.

Nichols, D.E. (1986) Differences between the mechanism of action of MDMA, MBDB, and the classic hallucinogens. Identification of a new therapeutic class: entactogens. Journal of psychoactive drugs, 18, 305-313.

Nichols, D.E., Lloyd, D.H., Hoffman, A.J., Nichols, M.B. \& Yim, G.K. (1982) Effects of certain hallucinogenic amphetamine analogues on the release of $[3 \mathrm{H}]$ serotonin from rat brain synaptosomes. Journal of medicinal chemistry, 25, 530-535. 
Nierenberg, A.A., Adler, L.A., Peselow, E., Zornberg, G. \& Rosenthal, M. (1994) Trazodone for antidepressant-associated insomnia. The American journal of psychiatry, 151, 1069-1072.

Nierenberg, A.A., Adler, L.A., Peselow, E., Zornberg, G. \& Rosenthal, M. (1994) Trazodone for antidepressant-associated insomnia. The American journal of psychiatry, 151, 1069-1072.

Norman, G., Brooks, S.P., Hennebry, G.M., Eacott, M.J. \& Little, H.J. (2002) Nimodipine prevents scopolamine-induced impairments in object recognition. J Psychopharmacol, 16, 153-161.

Nulsen, C.E., Fox, A.M. \& Hammond, G.R. (2010) Differential effects of ecstasy on short-term and working memory: a meta-analysis. Neuropsychology review, 20, 21-32.

O'Callaghan, J.P. \& Miller, D.B. (1993) Quantification of reactive gliosis as an approach to neurotoxicity assessment. NIDA research monograph, 136, $188-212$.

O'Callaghan, J.P. \& Miller, D.B. (1993) Quantification of reactive gliosis as an approach to neurotoxicity assessment. NIDA research monograph, 136, $188-212$.

Ogren, S.O., Eriksson, T.M., Elvander-Tottie, E., D'Addario, C., Ekstrom, J.C., Svenningsson, P., Meister, B., Kehr, J. \& Stiedl, O. (2008) The role of 5-HT(1A) receptors in learning and memory. Behavioural brain research, 195, 54-77.

O'Hearn, E., Battaglia, G., De Souza, E.B., Kuhar, M.J. \& Molliver, M.E. (1988) Methylenedioxyamphetamine (MDA) and methylenedioxymethamphetamine (MDMA) cause selective ablation of serotonergic axon terminals in forebrain: immunocytochemical evidence for neurotoxicity. J Neurosci, 8, 2788-2803.

Oliveri, M. \& Calvo, G. (2003) Increased visual cortical excitability in ecstasy users: a transcranial magnetic stimulation study. Journal of neurology, neurosurgery, and psychiatry, 74, 1136-1138.

Orejarena, M.J., Berrendero, F., Maldonado, R. \& Robledo, P. (2009) Differential changes in mesolimbic dopamine following contingent and non-contingent MDMA self-administration in mice. Psychopharmacology, 205, 457-466.

O'Shea, E., Granados, R., Esteban, B., Colado, M.I. \& Green, A.R. (1998) The relationship between the degree of neurodegeneration of rat brain 5-HT nerve terminals and the dose and frequency of administration of MDMA ('ecstasy'). Neuropharmacology, 37, 919926. 
O'Shea, E., Granados, R., Esteban, B., Colado, M.I. \& Green, A.R. (1998) The relationship between the degree of neurodegeneration of rat brain 5-HT nerve terminals and the dose and frequency of administration of MDMA ('ecstasy'). Neuropharmacology, 37, 919926.

O'Shea, E., Orio, L., Escobedo, I., Sanchez, V., Camarero, J., Green, A.R. \& Colado, M.I. (2006) MDMA-induced neurotoxicity: long-term effects on 5-HT biosynthesis and the influence of ambient temperature. British journal of pharmacology, 148, 778-785.

O'Shea, E., Orio, L., Escobedo, I., Sanchez, V., Camarero, J., Green, A.R. \& Colado, M.I. (2006) MDMA-induced neurotoxicity: long-term effects on 5-HT biosynthesis and the influence of ambient temperature. British journal of pharmacology, 148, 778-785.

Paladini, C.A., Mitchell, J.M., Williams, J.T. \& Mark, G.P. (2004) Cocaine self-administration selectively decreases noradrenergic regulation of metabotropic glutamate receptor-mediated inhibition in dopamine neurons. J Neurosci, 24, 5209-5215.

Parrott, A.C. (2001) Human psychopharmacology of Ecstasy (MDMA): a review of 15 years of empirical research. Human psychopharmacology, 16, 557-577.

Parrott, A.C. (2001) Human psychopharmacology of Ecstasy (MDMA): a review of 15 years of empirical research. Human psychopharmacology, 16, 557-577.

Pazos, A. \& Palacios, J.M. (1985) Quantitative autoradiographic mapping of serotonin receptors in the rat brain. I. Serotonin-1 receptors. Brain research, 346, 205-230.

Pazzagli, M., Giovannini, M.G. \& Pepeu, G. (1999) Trazodone increases extracellular serotonin levels in the frontal cortex of rats. European journal of pharmacology, 383, 249-257.

Pentney, A.R. (2001) An exploration of the history and controversies surrounding MDMA and MDA. Journal of psychoactive drugs, 33, 213-221.

Peroutka, S.J. (1987) Incidence of recreational use of 3,4methylenedimethoxymethamphetamine (MDMA, "ecstasy") on an undergraduate campus. The New England journal of medicine, 317, 1542-1543.

Perrine, S.A., Ghoddoussi, F., Michaels, M.S., Hyde, E.M., Kuhn, D.M. \& Galloway, M.P. MDMA administration decreases serotonin but not $\mathrm{N}$-acetylaspartate in the rat brain. Neurotoxicology, 31, 654-661. 
Perrine, S.A., Ghoddoussi, F., Michaels, M.S., Hyde, E.M., Kuhn, D.M. \& Galloway, M.P. (2010) MDMA administration decreases serotonin but not $\mathrm{N}$-acetylaspartate in the rat brain. Neurotoxicology, 31, 654661.

Peters, G.J. \& Kok, G. (2009) A structured review of reasons for ecstasy use and related behaviours: pointers for future research. BMC public health, 9, 230 .

Pickens, R. \& Harris, W.C. (1968) Self-administration of d-amphetamine by rats. Psychopharmacologia, 12, 158-163.

Pickens, R. \& Harris, W.C. (1968) Self-administration of d-amphetamine by rats. Psychopharmacologia, 12, 158-163.

Pineyro, G. \& Blier, P. (1999) Autoregulation of serotonin neurons: role in antidepressant drug action. Pharmacological reviews, 51, 533-591.

Pineyro, G. \& Blier, P. (1999) Autoregulation of serotonin neurons: role in antidepressant drug action. Pharmacological reviews, 51, 533-591.

Piper, B.J., Ali, S.F., Daniels, L.G. \& Meyer, J.S. Repeated intermittent methylenedioxymethamphetamine exposure protects against the behavioral and neurotoxic, but not hyperthermic, effects of an MDMA binge in adult rats. Synapse (New York, N.Y, 64, 421-431.

Piper, B.J., Ali, S.F., Daniels, L.G. \& Meyer, J.S. (2010) Repeated intermittent methylenedioxymethamphetamine exposure protects against the behavioral and neurotoxic, but not hyperthermic, effects of an MDMA binge in adult rats. Synapse (New York, N.Y, 64, 421431.

Piper, B.J., Fraiman, J.B. \& Meyer, J.S. (2005) Repeated MDMA ("Ecstasy") exposure in adolescent male rats alters temperature regulation, spontaneous motor activity, attention, and serotonin transporter binding. Developmental psychobiology, 47, 145-157.

Piper, B.J. \& Meyer, J.S. (2004) Memory deficit and reduced anxiety in young adult rats given repeated intermittent MDMA treatment during the periadolescent period. Pharmacology, biochemistry, and behavior, 79, 723-731.

Pizarro, N., Farre, M., Pujadas, M., Peiro, A.M., Roset, P.N., Joglar, J. \& de la Torre, R. (2004) Stereochemical analysis of 3,4methylenedioxymethamphetamine and its main metabolites in human samples including the catechol-type metabolite $(3,4-$ dihydroxymethamphetamine). Drug metabolism and disposition: the biological fate of chemicals, 32, 1001-1007. 
Pope, H.G., Jr., Ionescu-Pioggia, M. \& Pope, K.W. (2001) Drug use and life style among college undergraduates: a 30-year longitudinal study. The American journal of psychiatry, 158, 1519-1521.

Price, L.H., Ricaurte, G.A., Krystal, J.H. \& Heninger, G.R. (1989) Responses to i.v. L-tryptophan in MDMA users. NIDA research monograph, 95, 421-422.

Pubill, D., Canudas, A.M., Pallas, M., Camins, A., Camarasa, J. \& Escubedo, E. (2003) Different glial response to methamphetamineand methylenedioxymethamphetamine-induced neurotoxicity. Naunyn-Schmiedeberg's archives of pharmacology, 367, 490-499.

Pubill, D., Canudas, A.M., Pallas, M., Camins, A., Camarasa, J. \& Escubedo, E. (2003) Different glial response to methamphetamineand methylenedioxymethamphetamine-induced neurotoxicity. Naunyn-Schmiedeberg's archives of pharmacology, 367, 490-499.

Puma, C., Deschaux, O., Molimard, R. \& Bizot, J.C. (1999) Nicotine improves memory in an object recognition task in rats. European neuropsychopharmacology : the journal of the European College of Neuropsychopharmacology, 9, 323-327.

Quednow, B.B., Kuhn, K.U., Hoppe, C., Westheide, J., Maier, W., Daum, I. \& Wagner, M. (2007) Elevated impulsivity and impaired decisionmaking cognition in heavy users of MDMA ("Ecstasy"). Psychopharmacology, 189, 517-530.

Quinn, H.R., Matsumoto, I., Callaghan, P.D., Long, L.E., Arnold, J.C., Gunasekaran, N., Thompson, M.R., Dawson, B., Mallet, P.E., Kashem, M.A., Matsuda-Matsumoto, H., Iwazaki, T. \& McGregor, I.S. (2008) Adolescent rats find repeated Delta(9)-THC less aversive than adult rats but display greater residual cognitive deficits and changes in hippocampal protein expression following exposure. Neuropsychopharmacology, 33, 1113-1126.

Raffa, R.B., Shank, R.P. \& Vaught, J.L. (1992) Etoperidone, trazodone and MCPP: in vitro and in vivo identification of serotonin 5-HT1A (antagonistic) activity. Psychopharmacology, 108, 320-326.

Rahman, S., Zhang, J., Engleman, E.A. \& Corrigall, W.A. (2004) Neuroadaptive changes in the mesoaccumbens dopamine system after chronic nicotine self-administration: a microdialysis study. Neuroscience, 129, 415-424.

Ranaldi, R., Pocock, D., Zereik, R. \& Wise, R.A. (1999) Dopamine fluctuations in the nucleus accumbens during maintenance, extinction, and reinstatement of intravenous D-amphetamine selfadministration. J Neurosci, 19, 4102-4109. 
Rasmussen, T. \& Swedberg, M.D. (1998) Reinforcing effects of nicotinic compounds: intravenous self-administration in drug-naive mice. Pharmacology, biochemistry, and behavior, 60, 567-573.

Ratzenboeck, E., Saria, A., Kriechbaum, N. \& Zernig, G. (2001) Reinforcing effects of MDMA ("ecstasy") in drug-naive and cocaine-trained rats. Pharmacology, 62, 138-144.

Reichel, C.M., Schwendt, M., McGinty, J.F., Olive, M.F. \& See, R.E. (2011) Loss of object recognition memory produced by extended access to methamphetamine self-administration is reversed by positive allosteric modulation of metabotropic glutamate receptor 5 . Neuropsychopharmacology, 36, 782-792.

Reneman, L., Booij, J., de Bruin, K., Reitsma, J.B., de Wolff, F.A., Gunning, W.B., den Heeten, G.J. \& van den Brink, W. (2001) Effects of dose, sex, and long-term abstention from use on toxic effects of MDMA (ecstasy) on brain serotonin neurons. Lancet, 358, 1864-1869.

Reneman, L., Booij, J., Lavalaye, J., de Bruin, K., Reitsma, J.B., Gunning, B., den Heeten, G.J. \& van Den Brink, W. (2002) Use of amphetamine by recreational users of ecstasy (MDMA) is associated with reduced striatal dopamine transporter densities: a [123I]betaCIT SPECT study--preliminary report. Psychopharmacology, 159, 335-340.

Reneman, L., Booij, J., Schmand, B., van den Brink, W. \& Gunning, B. (2000) Memory disturbances in "Ecstasy" users are correlated with an altered brain serotonin neurotransmission. Psychopharmacology, 148, 322-324.

Reneman, L., Endert, E., de Bruin, K., Lavalaye, J., Feenstra, M.G., de Wolff, F.A. \& Booij, J. (2002) The acute and chronic effects of MDMA ("ecstasy") on cortical 5-HT2A receptors in rat and human brain. Neuropsychopharmacology, 26, 387-396.

Reneman, L., Lavalaye, J., Schmand, B., de Wolff, F.A., van den Brink, W., den Heeten, G.J. \& Booij, J. (2001) Cortical serotonin transporter density and verbal memory in individuals who stopped using 3,4methylenedioxymethamphetamine (MDMA or "ecstasy"): preliminary findings. Archives of general psychiatry, 58, 901-906.

Reneman, L., Majoie, C.B., Flick, H. \& den Heeten, G.J. (2002) Reduced $\mathrm{N}$-acetylaspartate levels in the frontal cortex of 3,4methylenedioxymethamphetamine (Ecstasy) users: preliminary results. AJNR. American journal of neuroradiology, 23, 231-237.

Reneman, L., Majoie, C.B., Habraken, J.B. \& den Heeten, G.J. (2001) Effects of ecstasy (MDMA) on the brain in abstinent users: initial 
observations with diffusion and perfusion MR imaging. Radiology, 220, 611-617.

Renoir, T., Paizanis, E., El Yacoubi, M., Saurini, F., Hanoun, N., Melfort, M., Lesch, K.P., Hamon, M. \& Lanfumey, L. (2008) Differential long-term effects of MDMA on the serotoninergic system and hippocampal cell proliferation in 5-HTT knock-out vs. wild-type mice. The international journal of neuropsychopharmacology / official scientific journal of the Collegium Internationale Neuropsychopharmacologicum (CINP), 11, 1149-1162.

Reveron, M.E., Maier, E.Y. \& Duvauchelle, C.L. (2006) Experiencedependent changes in temperature and behavioral activity induced by MDMA. Physiology \& behavior, 89, 358-363.

Reveron, M.E., Maier, E.Y. \& Duvauchelle, C.L. (2010) Behavioral, thermal and neurochemical effects of acute and chronic 3,4methylenedioxymethamphetamine ("Ecstasy") self-administration. Behavioural brain research, 207, 500-507.

Riad, M., Garcia, S., Watkins, K.C., Jodoin, N., Doucet, E., Langlois, X., el Mestikawy, S., Hamon, M. \& Descarries, L. (2000) Somatodendritic localization of 5-HT1A and preterminal axonal localization of 5HT1B serotonin receptors in adult rat brain. The Journal of comparative neurology, 417, 181-194.

Ricaurte, G.A., Finnegan, K.T., Irwin, I. \& Langston, J.W. (1990) Aminergic metabolites in cerebrospinal fluid of humans previously exposed to MDMA: preliminary observations. Annals of the New York Academy of Sciences, 600, 699-708; discussion 708-610.

Ricaurte, G.A., Forno, L.S., Wilson, M.A., DeLanney, L.E., Irwin, I., Molliver, M.E. \& Langston, J.W. (1988) (+/-)3,4Methylenedioxymethamphetamine selectively damages central serotonergic neurons in nonhuman primates. Jama, 260, 51-55.

Ricaurte, G.A., Markowska, A.L., Wenk, G.L., Hatzidimitriou, G., Wlos, J. \& Olton, D.S. (1993) 3,4-Methylenedioxymethamphetamine, serotonin and memory. The Journal of pharmacology and experimental therapeutics, 266, 1097-1105.

Ricaurte, G.A., Yuan, J. \& McCann, U.D. (2000) (+/-)3,4Methylenedioxymethamphetamine ('Ecstasy')-induced serotonin neurotoxicity: studies in animals. Neuropsychobiology, 42, 5-10.

Risner, M.E. (1975) Intravenous self-administration of d- and 1amphetamine by dog. European journal of pharmacology, 32, 344348. 
Risner, M.E. \& Goldberg, S.R. (1983) A comparison of nicotine and cocaine self-administration in the dog: fixed-ratio and progressiveratio schedules of intravenous drug infusion. The Journal of pharmacology and experimental therapeutics, 224, 319-326.

Roberts, G.M., Nestor, L. \& Garavan, H. (2009) Learning and memory deficits in ecstasy users and their neural correlates during a facelearning task. Brain research, 1292, 71-81.

Rodsiri, R., Spicer, C., Green, A.R., Marsden, C.A. \& Fone, K.C. (2011) Acute concomitant effects of MDMA binge dosing on extracellular 5-HT, locomotion and body temperature and the long-term effect on novel object discrimination in rats. Psychopharmacology, 213, 365376.

Rogers, G., Elston, J., Garside, R., Roome, C., Taylor, R., Younger, P., Zawada, A. \& Somerville, M. (2009) The harmful health effects of recreational ecstasy: a systematic review of observational evidence. Health Technol Assess, 13, iii-iv, ix-xii, 1-315.

Rogers, J.L., De Santis, S. \& See, R.E. (2008) Extended methamphetamine self-administration enhances reinstatement of drug seeking and impairs novel object recognition in rats. Psychopharmacology, 199, 615-624.

Rose, S.A., Gottfried, A.W. \& Bridger, W.H. (1983) Infants' cross-modal transfer from solid objects to their graphic representations. Child development, 54, 686-694.

Rothblat, L.A. \& Hayes, L.L. (1987) Short-term object recognition memory in the rat: nonmatching with trial-unique junk stimuli. Behavioral neuroscience, 101, 587-590.

Rothman, R.B., Baumann, M.H., Dersch, C.M., Romero, D.V., Rice, K.C., Carroll, F.I. \& Partilla, J.S. (2001) Amphetamine-type central nervous system stimulants release norepinephrine more potently than they release dopamine and serotonin. Synapse (New York, N.Y, 39, 32-41.

Rudnick, G. \& Wall, S.C. (1992) The molecular mechanism of "ecstasy" [3,4-methylenedioxy-methamphetamine (MDMA)]: serotonin transporters are targets for MDMA-induced serotonin release. Proceedings of the National Academy of Sciences of the United States of America, 89, 1817-1821.

Sabol, K.E., Lew, R., Richards, J.B., Vosmer, G.L. \& Seiden, L.S. (1996) Methylenedioxymethamphetamine-induced serotonin deficits are followed by partial recovery over a 52-week period. Part I: Synaptosomal uptake and tissue concentrations. The Journal of pharmacology and experimental therapeutics, 276, 846-854. 
Sabol, K.E., Lew, R., Richards, J.B., Vosmer, G.L. \& Seiden, L.S. (1996) Methylenedioxymethamphetamine-induced serotonin deficits are followed by partial recovery over a 52-week period. Part I: Synaptosomal uptake and tissue concentrations. The Journal of pharmacology and experimental therapeutics, 276, 846-854.

Sabol, K.E. \& Seiden, L.S. (1998) Reserpine attenuates D-amphetamine and MDMA-induced transmitter release in vivo: a consideration of dose, core temperature and dopamine synthesis. Brain research, 806, 6978 .

Sanchez, S., Paredes, S.D., Sanchez, C.L., Barriga, C., Reiter, R.J. \& Rodriguez, A.B. (2008) Tryptophan administration in rats enhances phagocytic function and reduces oxidative metabolism. Neuro endocrinology letters, 29, 1026-1032.

Sastre-Coll, A., Esteban, S. \& Garcia-Sevilla, J.A. (2002) Supersensitivity of 5-HT1A autoreceptors and alpha2-adrenoceptors regulating monoamine synthesis in the brain of morphine-dependent rats. Naunyn-Schmiedeberg's archives of pharmacology, 365, 210-219.

Sastre-Coll, A., Esteban, S. \& Garcia-Sevilla, J.A. (2002) Supersensitivity of 5-HT1A autoreceptors and alpha2-adrenoceptors regulating monoamine synthesis in the brain of morphine-dependent rats. Naunyn-Schmiedeberg's archives of pharmacology, 365, 210-219.

Scali, C., Giovannini, M.G., Bartolini, L., Prosperi, C., Hinz, V., Schmidt, B. \& Pepeu, G. (1997) Effect of metrifonate on extracellular brain acetylcholine and object recognition in aged rats. European journal of pharmacology, 325, 173-180.

Scanzello, C.R., Hatzidimitriou, G., Martello, A.L., Katz, J.L. \& Ricaurte, G.A. (1993) Serotonergic recovery after (+/-)3,4-(methylenedioxy) methamphetamine injury: observations in rats. The Journal of pharmacology and experimental therapeutics, 264, 1484-1491.

Scanzello, C.R., Hatzidimitriou, G., Martello, A.L., Katz, J.L. \& Ricaurte, G.A. (1993) Serotonergic recovery after (+/-)3,4-(methylenedioxy) methamphetamine injury: observations in rats. The Journal of pharmacology and experimental therapeutics, 264, 1484-1491.

Scanzello, C.R., Hatzidimitriou, G., Martello, A.L., Katz, J.L. \& Ricaurte, G.A. (1993) Serotonergic recovery after (+/-)3,4-(methylenedioxy) methamphetamine injury: observations in rats. The Journal of pharmacology and experimental therapeutics, 264, 1484-1491.

Schenk, S. (2009) MDMA self-administration in laboratory animals: a summary of the literature and proposal for future research. Neuropsychobiology, 60, 130-136. 
Schenk, S. (2011) MDMA ("ecstasy") abuse as an example of dopamine neuroplasticity. Neuroscience and biobehavioral reviews, 35, 12031218.

Schenk, S., Gittings, D. \& Colussi-Mas, J. Dopaminergic mechanisms of reinstatement of MDMA-seeking behaviour in rats. British journal of pharmacology, 162, 1770-1780.

Schenk, S., Gittings, D., Johnstone, M. \& Daniela, E. (2003) Development, maintenance and temporal pattern of self-administration maintained by ecstasy (MDMA) in rats. Psychopharmacology, 169, 21-27.

Schenk, S., Harper, D.N. \& Do, J. (2011) Novel object recognition memory: measurement issues and effects of MDMA self-administration following short inter-trial intervals. J Psychopharmacol, 25, 10431052 .

Schenk, S., Hely, L., Lake, B., Daniela, E., Gittings, D. \& Mash, D.C. (2007) MDMA self-administration in rats: acquisition, progressive ratio responding and serotonin transporter binding. The European journal of neuroscience, 26, 3229-3236.

Schenk, S., Hely, L., Lake, B., Daniela, E., Gittings, D. \& Mash, D.C. (2007) MDMA self-administration in rats: acquisition, progressive ratio responding and serotonin transporter binding. The European journal of neuroscience, 26, 3229-3236.

Schenk, S. \& Partridge, B. (1997) Sensitization and tolerance in psychostimulant self-administration. Pharmacology, biochemistry, and behavior, 57, 543-550.

Schifano, F., Di Furia, L., Forza, G., Minicuci, N. \& Bricolo, R. (1998) MDMA ('ecstasy') consumption in the context of polydrug abuse: a report on 150 patients. Drug and alcohol dependence, 52, 85-90.

Schilt, T., Koeter, M.W., Smal, J.P., Gouwetor, M.N., van den Brink, W. \& Schmand, B. (2010) Long-term neuropsychological effects of ecstasy in middle-aged ecstasy/polydrug users. Psychopharmacology, 207, 583-591.

Schmidt, C.J. (1987) Acute administration of methylenedioxymethamphetamine: comparison with the neurochemical effects of its $\mathrm{N}$-desmethyl and $\mathrm{N}$-ethyl analogs. European journal of pharmacology, 136, 81-88.

Schmidt, C.J., Levin, J.A. \& Lovenberg, W. (1987) In vitro and in vivo neurochemical effects of methylenedioxymethamphetamine on striatal monoaminergic systems in the rat brain. Biochemical pharmacology, 36, 747-755. 
Schmidt, C.J. \& Taylor, V.L. (1987) Depression of rat brain tryptophan hydroxylase activity following the acute administration of methylenedioxymethamphetamine. Biochemical pharmacology, 36, 4095-4102.

Schmidt, C.J. \& Taylor, V.L. (1988) Direct central effects of acute methylenedioxymethamphetamine on serotonergic neurons. European journal of pharmacology, 156, 121-131.

Schmidt, C.J., Wu, L. \& Lovenberg, W. (1986) Methylenedioxymethamphetamine: a potentially neurotoxic amphetamine analogue. European journal of pharmacology, 124, 175-178.

Schmued, L.C. (2003) Demonstration and localization of neuronal degeneration in the rat forebrain following a single exposure to MDMA. Brain research, 974, 127-133.

Schneider, M., Schomig, E. \& Leweke, F.M. (2008) Acute and chronic cannabinoid treatment differentially affects recognition memory and social behavior in pubertal and adult rats. Addiction biology, 13, 345-357.

Schuster, C.R. \& Thompson, T. (1969) Self administration of and behavioral dependence on drugs. Annual review of pharmacology, $\mathbf{9}$, 483-502.

Selvaraj, S., Hoshi, R., Bhagwagar, Z., Murthy, N.V., Hinz, R., Cowen, P., Curran, H.V. \& Grasby, P. (2009) Brain serotonin transporter binding in former users of MDMA ('ecstasy'). The British journal of psychiatry : the journal of mental science, 194, 355-359.

Semple, D.M., Ebmeier, K.P., Glabus, M.F., O'Carroll, R.E. \& Johnstone, E.C. (1999) Reduced in vivo binding to the serotonin transporter in the cerebral cortex of MDMA ('ecstasy') users. The British journal of psychiatry : the journal of mental science, 175, 63-69.

Sharkey, J., McBean, D.E. \& Kelly, P.A. (1991) Alterations in hippocampal function following repeated exposure to the amphetamine derivative methylenedioxymethamphetamine

Psychopharmacology, 105, 113-118.

("Ecstasy").

Siegel, R.K. (1986) MDMA. Nonmedical use and intoxication. Journal of psychoactive drugs, 18, 349-354.

Silber, B.Y. \& Schmitt, J.A. (2010) Effects of tryptophan loading on human cognition, mood, and sleep. Neuroscience and biobehavioral reviews, 34, 387-407. 
Sitte, H., Edelmann, L., Hassig, H., Kleber, H. \& Lang, A. (1994) A new versatile system for freeze-substitution, freeze-drying and low temperature embedding of biological specimens. Scanning microscopy. Supplement, 8, 47-64; discussion 64-46.

Sizemore, G.M., Co, C. \& Smith, J.E. (2000) Ventral pallidal extracellular fluid levels of dopamine, serotonin, gamma amino butyric acid, and glutamate during cocaine self-administration in rats. Psychopharmacology, 150, 391-398.

Skelton, M.R., Able, J.A., Grace, C.E., Herring, N.R., Schaefer, T.L., Gudelsky, G.A., Vorhees, C.V. \& Williams, M.T. (2008) (+/-)-3,4Methylenedioxymethamphetamine treatment in adult rats impairs path integration learning: a comparison of single vs once per week treatment for 5 weeks. Neuropharmacology, 55, 1121-1130.

Skelton, M.R., Williams, M.T. \& Vorhees, C.V. (2006) Treatment with MDMA from P11-20 disrupts spatial learning and path integration learning in adolescent rats but only spatial learning in older rats. Psychopharmacology, 189, 307-318.

Smith, B.R., Brown, Z.W. \& Amit, Z. (1982) Intraventricular selfadministration of leucine-enkephalin by laboratory rats. Life sciences, 31, 1527-1530.

Sprague, J.E., Huang, X., Kanthasamy, A. \& Nichols, D.E. (1994) Attenuation of 3,4-methylenedioxymethamphetamine (MDMA) induced neurotoxicity with the serotonin precursors tryptophan and 5-hydroxytryptophan. Life sciences, 55, 1193-1198.

Sprague, J.E., Preston, A.S., Leifheit, M. \& Woodside, B. (2003) Hippocampal serotonergic damage induced by MDMA (ecstasy): effects on spatial learning. Physiology \& behavior, 79, 281-287.

Sprouse, J.S. \& Aghajanian, G.K. (1987) Electrophysiological responses of serotoninergic dorsal raphe neurons to 5-HT1A and 5-HT1B agonists. Synapse (New York, N.Y, 1, 3-9.

Stahl, S.M. (1998) Mechanism of action of serotonin selective reuptake inhibitors. Serotonin receptors and pathways mediate therapeutic effects and side effects. Journal of affective disorders, 51, 215-235.

Stanley, N., Salem, A. \& Irvine, R.J. (2007) The effects of coadministration of 3,4-methylenedioxymethamphetamine ("ecstasy") or para-methoxyamphetamine and moclobemide at elevated ambient temperatures on striatal 5-HT, body temperature and behavior in rats. Neuroscience, 146, 321-329.

Stefanski, R., Justinova, Z., Hayashi, T., Takebayashi, M., Goldberg, S.R. \& $\mathrm{Su}$, T.P. (2004) Sigmal receptor upregulation after chronic 
methamphetamine self-administration in rats: a study with yoked controls. Psychopharmacology, 175, 68-75.

Stefanski, R., Ladenheim, B., Lee, S.H., Cadet, J.L. \& Goldberg, S.R. (1999) Neuroadaptations in the dopaminergic system after active selfadministration but not after passive administration of methamphetamine. European journal of pharmacology, 371, 123135.

Stone, D.M., Johnson, M., Hanson, G.R. \& Gibb, J.W. (1988) Role of endogenous dopamine in the central serotonergic deficits induced by 3,4-methylenedioxymethamphetamine. The Journal of pharmacology and experimental therapeutics, 247, 79-87.

Stone, D.M., Johnson, M., Hanson, G.R. \& Gibb, J.W. (1989) Acute inactivation of tryptophan hydroxylase by amphetamine analogs involves the oxidation of sulfhydryl sites. European journal of pharmacology, 172, 93-97.

Stone, D.M., Merchant, K.M., Hanson, G.R. \& Gibb, J.W. (1987) Immediate and long-term effects of 3,4methylenedioxymethamphetamine on serotonin pathways in brain of rat. Neuropharmacology, 26, 1677-1683.

Stone, D.M., Stahl, D.C., Hanson, G.R. \& Gibb, J.W. (1986) The effects of 3,4-methylenedioxymethamphetamine (MDMA) and 3,4methylenedioxyamphetamine (MDA) on monoaminergic systems in the rat brain. European journal of pharmacology, 128, 41-48.

Taffe, M.A., Weed, M.R., Davis, S., Huitron-Resendiz, S., Schroeder, R., Parsons, L.H., Henriksen, S.J. \& Gold, L.H. (2001) Functional consequences of repeated (+/-)3,4methylenedioxymethamphetamine (MDMA) treatment in rhesus monkeys. Neuropsychopharmacology, 24, 230-239.

Tancer, M. \& Johanson, C.E. (2007) The effects of fluoxetine on the subjective and physiological effects of 3,4methylenedioxymethamphetamine (MDMA) in humans. Psychopharmacology, 189, 565-573.

Thomasius, R., Petersen, K., Buchert, R., Andresen, B., Zapletalova, P., Wartberg, L., Nebeling, B. \& Schmoldt, A. (2003) Mood, cognition and serotonin transporter availability in current and former ecstasy (MDMA) users. Psychopharmacology, 167, 85-96.

Thomasius, R., Zapletalova, P., Petersen, K., Buchert, R., Andresen, B., Wartberg, L., Nebeling, B. \& Schmoldt, A. (2006) Mood, cognition and serotonin transporter availability in current and former ecstasy (MDMA) users: the longitudinal perspective. J Psychopharmacol, 20, 211-225. 
Thompson, M.R., Li, K.M., Clemens, K.J., Gurtman, C.G., Hunt, G.E., Cornish, J.L. \& McGregor, I.S. (2004) Chronic fluoxetine treatment partly attenuates the long-term anxiety and depressive symptoms induced by MDMA ('Ecstasy') in rats. Neuropsychopharmacology, 29, 694-704.

Trigo, J.M., Panayi, F., Soria, G., Maldonado, R. \& Robledo, P. (2006) A reliable model of intravenous MDMA self-administration in naive mice. Psychopharmacology, 184, 212-220.

Trulson, M.E. \& Trulson, V.M. (1982) Effects of chronic methamphetamine administration on tryptophan hydroxylase activity, [3H]serotonin synaptosomal uptake, and serotonin metabolism in rat brain following systemic tryptophan loading. Neuropharmacology, 21, 521-527.

Tucker, G.T., Lennard, M.S., Ellis, S.W., Woods, H.F., Cho, A.K., Lin, L.Y., Hiratsuka, A., Schmitz, D.A. \& Chu, T.Y. (1994) The demethylenation of methylenedioxymethamphetamine ("ecstasy") by debrisoquine hydroxylase (CYP2D6). Biochemical pharmacology, 47, 1151-1156.

Upreti, V.V. \& Eddington, N.D. (2008) Fluoxetine pretreatment effects pharmacokinetics of 3,4-methylenedioxymethamphetamine (MDMA, ECSTASY) in rat. Journal of pharmaceutical sciences, 97, 15931605.

Vannucchi, M.G., Scali, C., Kopf, S.R., Pepeu, G. \& Casamenti, F. (1997) Selective muscarinic antagonists differentially affect in vivo acetylcholine release and memory performances of young and aged rats. Neuroscience, $\mathbf{7 9}, 837-846$.

Verdejo-Garcia, A.J., Lopez-Torrecillas, F., Aguilar de Arcos, F. \& PerezGarcia, M. (2005) Differential effects of MDMA, cocaine, and cannabis use severity on distinctive components of the executive functions in polysubstance users: a multiple regression analysis. Addictive behaviors, 30, 89-101.

Verheyden, S.L., Henry, J.A. \& Curran, H.V. (2003) Acute, sub-acute and long-term subjective consequences of 'ecstasy' (MDMA) consumption in 430 regular users. Human psychopharmacology, 18, 507-517.

Vorhees, C.V., Reed, T.M., Skelton, M.R. \& Williams, M.T. (2004) Exposure to 3,4-methylenedioxymethamphetamine (MDMA) on postnatal days 11-20 induces reference but not working memory deficits in the Morris water maze in rats: implications of prior learning. International journal of developmental neuroscience : the 
official journal of the International Society for Developmental Neuroscience, 22, 247-259.

Vuori, E., Henry, J.A., Ojanpera, I., Nieminen, R., Savolainen, T., Wahlsten, P. \& Jantti, M. (2003) Death following ingestion of MDMA (ecstasy) and moclobemide. Addiction, 98, 365-368.

Wang, X., Baumann, M.H., Dersch, C.M. \& Rothman, R.B. (2007) Restoration of 3,4-methylenedioxymethamphetamine-induced 5-HT depletion by the administration of L-5-hydroxytryptophan. Neuroscience, 148, 212-220.

Wang, X., Baumann, M.H., Xu, H., Morales, M. \& Rothman, R.B. (2005) (+/-)-3,4-Methylenedioxymethamphetamine administration to rats does not decrease levels of the serotonin transporter protein or alter its distribution between endosomes and the plasma membrane. The Journal of pharmacology and experimental therapeutics, 314, 10021012.

Wang, X., Baumann, M.H., Xu, H. \& Rothman, R.B. (2004) 3,4methylenedioxymethamphetamine (MDMA) administration to rats decreases brain tissue serotonin but not serotonin transporter protein and glial fibrillary acidic protein. Synapse (New York, N.Y, 53, 240248.

Wang, X., Baumann, M.H., Xu, H. \& Rothman, R.B. (2004) 3,4methylenedioxymethamphetamine (MDMA) administration to rats decreases brain tissue serotonin but not serotonin transporter protein and glial fibrillary acidic protein. Synapse (New York, N.Y, 53, 240248.

Wareing, M., Fisk, J.E., Murphy, P. \& Montgomery, C. (2004) Verbal working memory deficits in current and previous users of MDMA. Human psychopharmacology, 19, 225-234.

Warren, M.W., Zheng, W., Kobeissy, F.H., Cheng Liu, M., Hayes, R.L., Gold, M.S., Larner, S.F. \& Wang, K.K. (2007) Calpain- and caspase-mediated alphaII-spectrin and tau proteolysis in rat cerebrocortical neuronal cultures after ecstasy or methamphetamine exposure. The international journal of neuropsychopharmacology / official scientific journal of the Collegium Internationale Neuropsychopharmacologicum (CINP), 10, 479-489.

Watkins, S.S., Epping-Jordan, M.P., Koob, G.F. \& Markou, A. (1999) Blockade of nicotine self-administration with nicotinic antagonists in rats. Pharmacology, biochemistry, and behavior, 62, 743-751.

Weeks, J.R. (1962) Experimental morphine addiction: method for automatic intravenous injections in unrestrained rats. Science, 138, 143-144. 
Weeks, J.R. \& Collins, R.J. (1964) Factors affecting voluntary morphine intake in self-maintained addicted rats. Psychopharmacologia, 6, 267-279.

White, B., Degenhardt, L., Breen, C., Bruno, R., Newman, J. \& Proudfoot, P. (2006) Risk and benefit perceptions of party drug use. Addictive behaviors, 31, 137-142.

Wilkins, C. \& Sweetsur, P. (2008) Trends in population drug use in New Zealand: findings from national household surveying of drug use in 1998, 2001, 2003, and 2006. The New Zealand medical journal, 121, 61-71.

Wilson, M.C., Hitomi, M. \& Schuster, C.R. (1971) Psychomotor stimulant self administration as a function of dosage per injection in the rhesus monkey. Psychopharmacologia, 22, 271-281.

Winnicka, M.M. \& Braszko, J.J. (1997) 6-OHDA lesions to the central amygdala abolish angiotensins facilitation of object recognition in rats. General pharmacology, 29, 239-243.

Winstock, A.R., Wolff, K. \& Ramsey, J. (2001) Ecstasy pill testing: harm minimization gone too far? Addiction, 96, 1139-1148.

Winters, B.D., Saksida, L.M. \& Bussey, T.J. (2008) Object recognition memory: neurobiological mechanisms of encoding, consolidation and retrieval. Neuroscience and biobehavioral reviews, 32, 10551070 .

Wise, R.A., Newton, P., Leeb, K., Burnette, B., Pocock, D. \& Justice, J.B., Jr. (1995) Fluctuations in nucleus accumbens dopamine concentration during intravenous cocaine self-administration in rats. Psychopharmacology, 120, 10-20.

Wood, J.L. \& Russo, A.F. (2001) Autoregulation of cell-specific MAP kinase control of the tryptophan hydroxylase promoter. The Journal of biological chemistry, 276, 21262-21271.

Wu, P., Liu, X., Pham, T.H., Jin, J., Fan, B. \& Jin, Z. (2010) Ecstasy use among US adolescents from 1999 to 2008. Drug and alcohol dependence, 112, 33-38.

Xie, T., Tong, L., McLane, M.W., Hatzidimitriou, G., Yuan, J., McCann, U. \& Ricaurte, G. (2006) Loss of serotonin transporter protein after MDMA and other ring-substituted amphetamines. Neuropsychopharmacology, 31, 2639-2651.

Yamamoto, B.K. \& Spanos, L.J. (1988) The acute effects of methylenedioxymethamphetamine on dopamine release in the 
awake-behaving rat. European journal of pharmacology, 148, 195203.

Yokel, R.A. \& Pickens, R. (1973) Self-administration of optical isomers of amphetamine and methylamphetamine by rats. The Journal of pharmacology and experimental therapeutics, 187, 27-33.

Yokel, R.A. \& Pickens, R. (1974) Drug level of d- and l-amphetamine during intravenous self-administration. Psychopharmacologia, 34, 255-264.

You, Z.B., Wang, B., Zitzman, D., Azari, S. \& Wise, R.A. (2007) A role for conditioned ventral tegmental glutamate release in cocaine seeking. J Neurosci, 27, 10546-10555.

You, Z.B., Wang, B., Zitzman, D. \& Wise, R.A. (2008) Acetylcholine release in the mesocorticolimbic dopamine system during cocaine seeking: conditioned and unconditioned contributions to reward and motivation. J Neurosci, 28, 9021-9029.

Young, S.N. \& Gauthier, S. (1981) Tryptophan availability and the control of 5-hydroxytryptamine and tryptamine synthesis in human CNS. Advances in experimental medicine and biology, 133, 221-230.

Zakzanis, K.K., Campbell, Z. \& Jovanovski, D. (2007) The neuropsychology of ecstasy (MDMA) use: a quantitative review. Human psychopharmacology, 22, 427-435.

Zakzanis, K.K. \& Young, D.A. (2001) Executive function in abstinent MDMA ('ecstasy') users. Medical science monitor: international medical journal of experimental and clinical research, 7, 1292-1298.

Zakzanis, K.K., Young, D.A. \& Radkhoshnoud, N.F. (2002) Attentional processes in abstinent methylenedioxymethamphetamine (ecstasy) users. Applied neuropsychology, 9, 84-91. 